\title{
The signal transduction of synapse formation and its failure in Rett syndrome.
}

\author{
Dissertation \\ for the award of the degree \\ "Doctor rerum naturalium" \\ Division of Mathematics and Natural Sciences \\ Of the Georg-August-Universität Göttingen
}

within the doctoral program Molecular Physiology of the Brain of the Georg-August University School of Science (GAUSS)

submitted by

René Ebrecht

from Magdeburg, Germany

Göttingen, 2016 


\section{Thesis Committee}

\section{Prof. Dr. Fred Wouters}

University Medical Center Göttingen, Institute for Neuropathology.

\section{Dr. Till Marquardt}

European Neuroscience Institute Göttingen, Developmental Neurobiology Group

\section{Prof. Dr. Jörg Enderlein}

III. Physical Institute, Biophysics / Complex Systems

\section{Examination Board}

Prof. Dr. Nils Brose

Max Planck Institute for Experimental Medicine, Department of Molecular Neurobiology

Prof. Dr. Thomas Dresbach

University Medical Center Göttingen Center of Anatomy, Department of Anatomy and Embryology

Prof. Dr. Michael Hörner

Johann-Friedrich-Blumenbach Institute for Zoology and Anthropology, Department of Cellular Neurobiology 


\section{Disclaimer}

Sections 1.6 "FRET" and 1.7 "FLIM" of Chapter 1 "Introduction" containing Figure 4 and Figure 5 were published as a part of a review article in the Journal Protoplasma entitled "Fluorescence lifetime imaging microscopy in the medical sciences." by René Ebrecht, Craig Don Paul and Fred Wouters (Ebrecht R, Don Paul C, Wouters FS (2014) Fluorescence lifetime imaging microscopy in the medical sciences. Protoplasma. 2014 Mar;251(2):293-305. These sections include "Time domain FLIM techniques", TD FLIM image analysis" and "Frequency Domain FLIM techniques" and are reproduced in this document in an altered version.

\section{General Declaration}

I hereby declare that my doctoral thesis "The signal transduction of synapse formation and its failure in Rett syndrome" has been written independently, with no other sources and aids, other than quoted. This thesis has not been submitted elsewhere for any academic degree.

Göttingen, January 2016

René Ebrecht 


\section{Table of Contents}

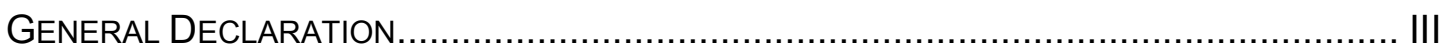

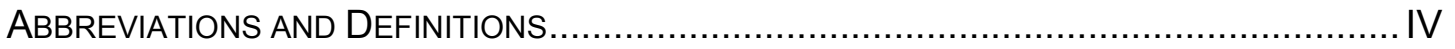

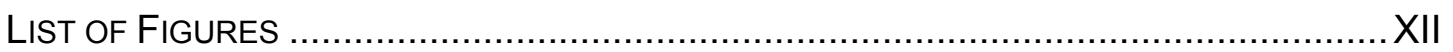

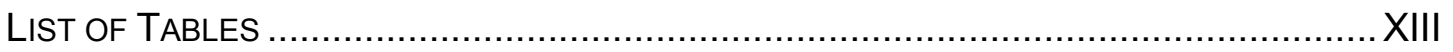

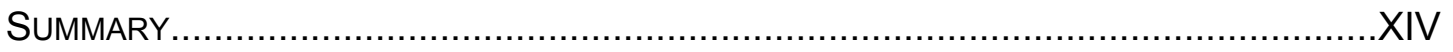

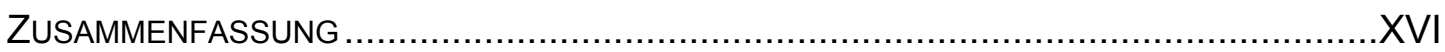

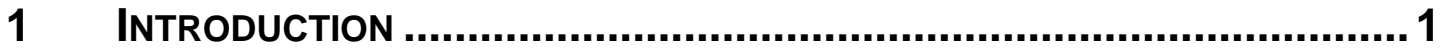

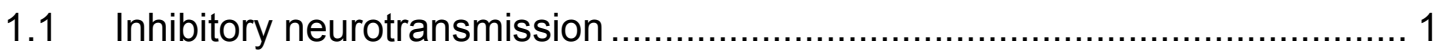

1.2 Gephyrin

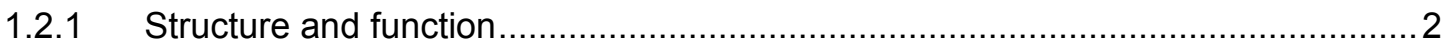

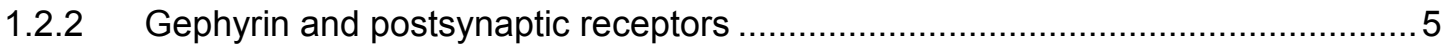

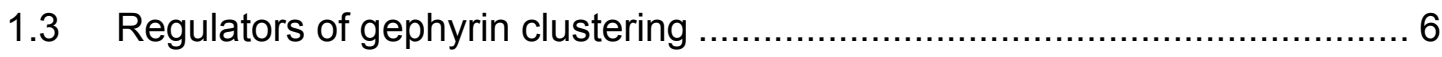

1.3.1 Neuroligins

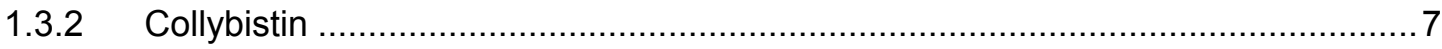

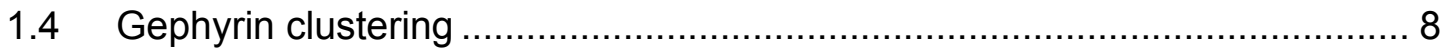

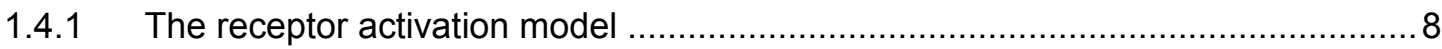

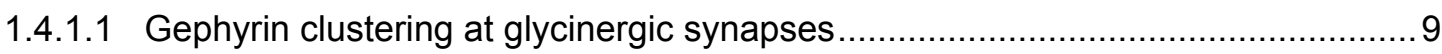

1.4.1.2 Gephyrin clustering at GABAergic synapses ………....................................

1.4.2 Gephyrin phosphorylation in GABAergic synapse plasticity ..............................11

1.4.2.1 GSK-3 $\beta$ and CDK5 dependent phosphorylation of serine $270 \ldots \ldots \ldots \ldots \ldots \ldots \ldots \ldots \ldots . .12$

1.4.2.2 ERK1/2 dependent phosphorylation of serine $268 \ldots \ldots \ldots \ldots \ldots \ldots \ldots \ldots \ldots \ldots \ldots \ldots \ldots \ldots . . . .13$ 


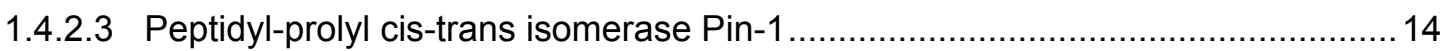

1.4.2.4 Other post-translational modifications of gephyrin .................................... 15

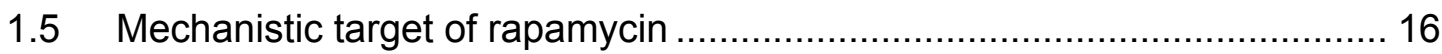

1.5.1 Structure function regulation of the mTOR complexes ................................... 17

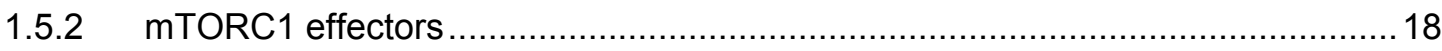

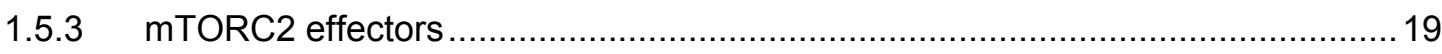

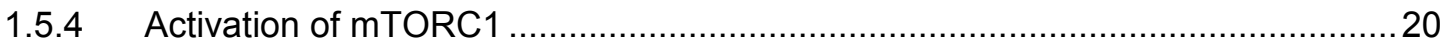

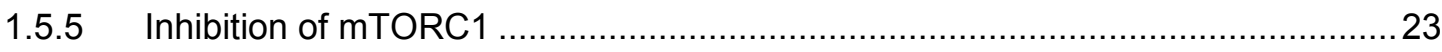

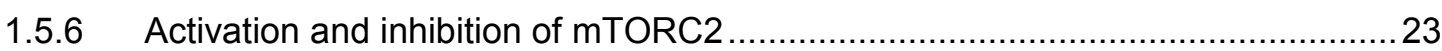

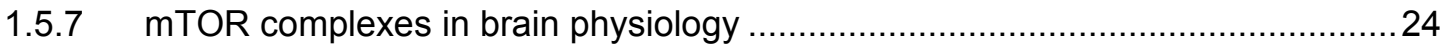

1.5.7.1 Dysregulation of mTOR in neurodevelopmental disease................................24

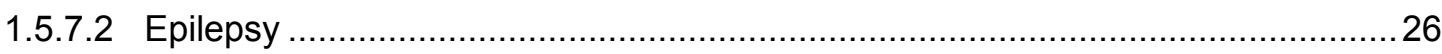

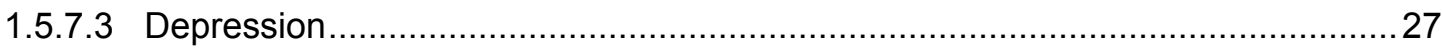

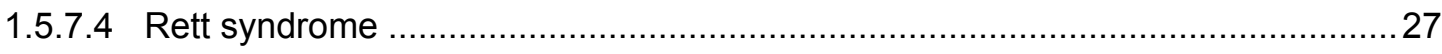

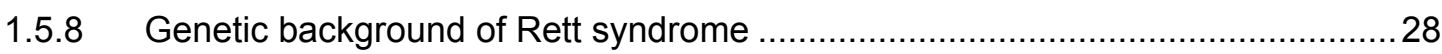

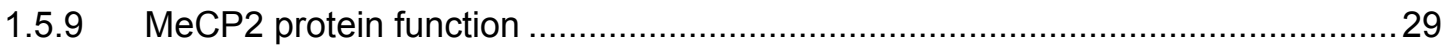

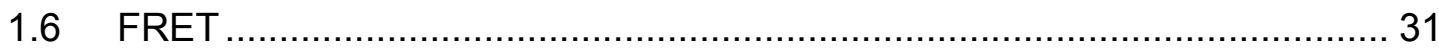

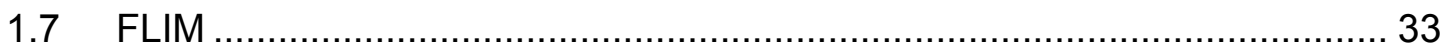

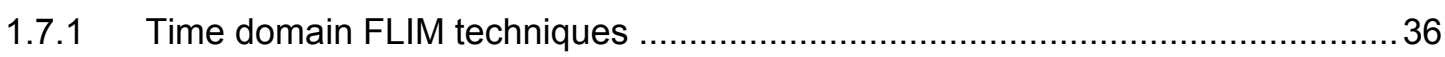

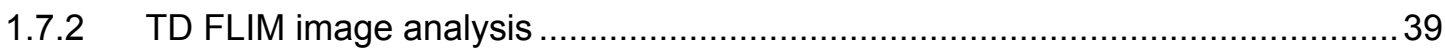

1.7.3 Frequency Domain FLIM techniques ..................................................... 40 


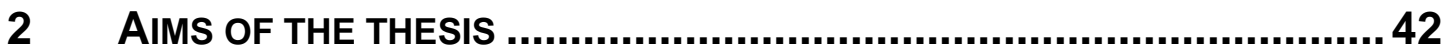

3 MATERIALS AND METHOdS......................................................... 43

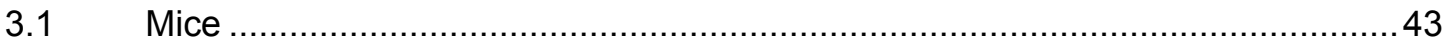

3.1.1 The MeCP2 ${ }^{\text {tml.IBird }} \mathrm{KO}$ mouse as a model of Rett syndrome...........................43

3.1.1.1 DNA isolation and genotyping of MeCP2 ${ }^{\text {tml.IBird }} \mathrm{KO}$ mouse ............................ 43

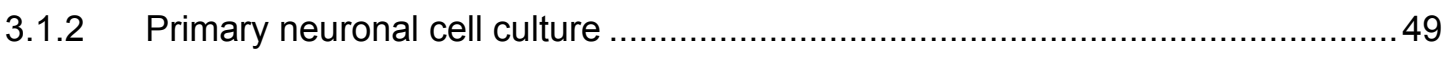

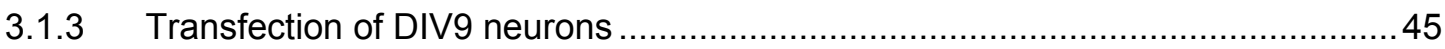

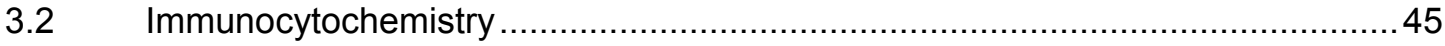

3.3 Antibody labelling with amine-reactive ATTO-labels.................................. 47

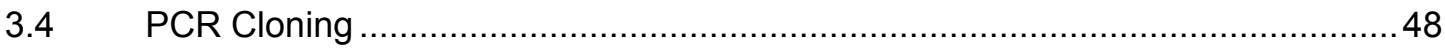

3.5 Cloning of C-terminally labelled Gephyrin constructs .....................................49

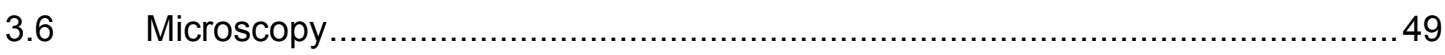

3.7 Image Analysis, Quantification and Representation ..................................... 50

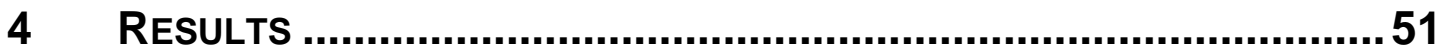

4.1 Establishment of antibody-based phosphorylation assays ..................... 51

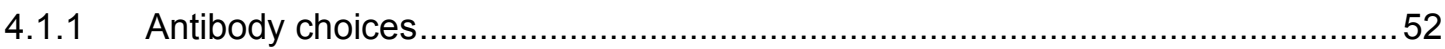

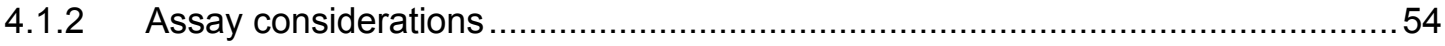

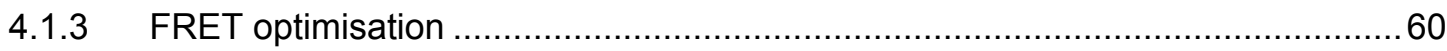

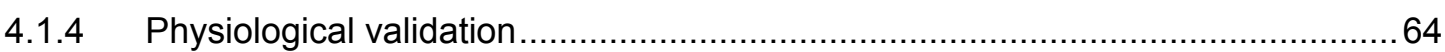

4.1.5 Monovalent Fab fragments in antibody-based FRET assay ............................68

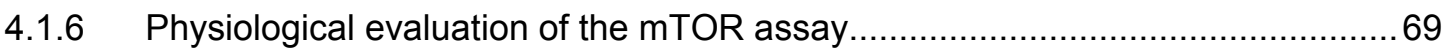

4.1.7 mTORC1 signalling in MeCP2-KO neurons............................................ 73

4.2 Development of a mTORC2 activity assay ...................................... 75 
4.2.1 Application of the Akt phosphorylation assay to MeCP2-KO neurons. 82

4.3 A FRET-based mTOR-gephyrin interaction assay .............................. 84

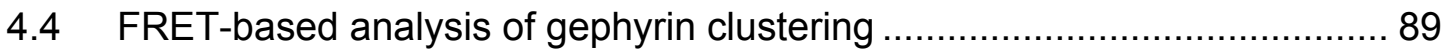

4.5 Gephyrin trimerisation in GABAergic postsynapses ................................ 96

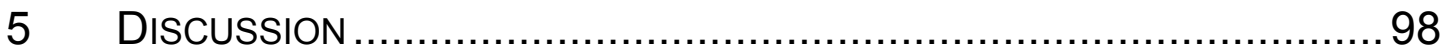

5.1 FRET-based mTORC1 and mTORC2 activity assays ............................ 98

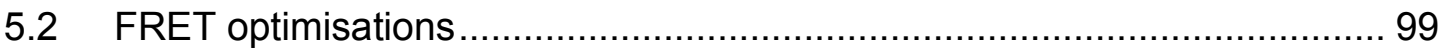

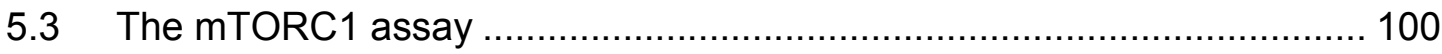

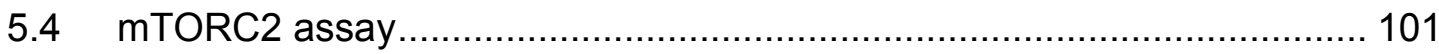

5.5 Compromised mTORC1 and mTORC2 signalling in Rett syndrome. ....... 101

5.6 mTOR-gephyrin interaction ............................................................... 103

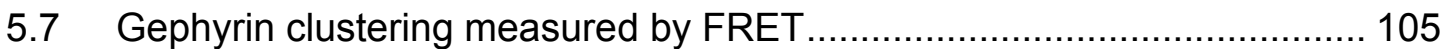

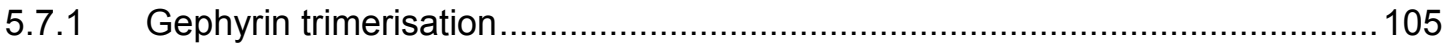

5.7.2 Gephyrin trimerisation and dimerization mutants ........................................ 105

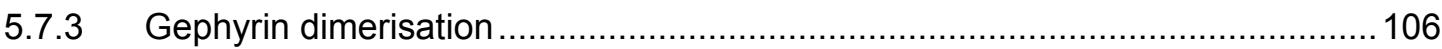

6 Conclusions and Future Perspectives .................................. 107

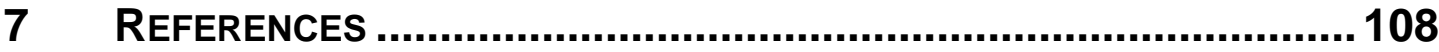

ACKNOWLEDGEMENTS................................................................. 120 


\section{Abbreviations and Definitions}

This is a consolidated list of all abbreviations used in this thesis including those found within the published papers.

\begin{tabular}{ll} 
A & Alanine \\
AMPK & 5'AMP activated protein kinase \\
ASD & Amyloid $\beta$ protein precursor \\
BDNF & Autism spectrum disorders \\
CAMP & Brain derived neurotrophic factor \\
CB & Cyclic adenosine monophosphate \\
CB1-3 & Collybistin \\
CBD & Collybistin isoforms-1 - 3 \\
CCD & Collybistin binding domain \\
CDC42 & Charge-coupled device \\
CDK5 & Cell division control protein 42 \\
CREB1 & Cyclin dependent kinase 5 \\
DEPTOR & CAMP response element binding protein \\
DH & DEP domain containing mTOR interacting protein \\
DGC & DBL homology \\
DMEM & Dystrophin-glycoprotein complex \\
E & Dulbecco's modified Eagle's medium \\
eEF2K & FRET efficiency \\
eIF4E & Eukaryotic elongation factor-2 kinase \\
ERK1/2 & Eukaryotic translation initiation factor 4E \\
FAT & FRAP, ATM, TRAP \\
\hline
\end{tabular}




\begin{tabular}{|c|c|}
\hline FCS & Foetal calf serum \\
\hline FD & Frequency domain \\
\hline FLIM & Fluorescence lifetime imaging microscopy \\
\hline FoxO & Forkhead box $\mathrm{O}$ \\
\hline FRET & Förster resonance energy transfer \\
\hline fs & femto second \\
\hline GABA & Gamma amino butyric acid \\
\hline $\mathrm{GABA}_{\mathrm{A}}$ & Type A GABA receptor \\
\hline GEF & Guanosine triphosphate exchange factor \\
\hline GlyR & Glycine receptor \\
\hline GPCRs & G-protein coupled receptors \\
\hline GSK-3ß & Glycongen synthase kinase $3 \beta$ \\
\hline HDAC & Histone deacetylase \\
\hline HEAT & Huntingtin, EF3, PP2A, TOR1 \\
\hline HEK293 & Human embryonic kidney cells 293 \\
\hline HIF1a & Hypoxia inducible factor $1 \alpha$ \\
\hline I & Isoleucine \\
\hline IRF & Instrument response function \\
\hline IRS & Insulin receptor substrate \\
\hline IKKB & IKB kinase $\beta$ \\
\hline$\kappa^{2}$ & Dipole orientation factor \\
\hline KO & Knockout \\
\hline L & Lysine \\
\hline LTD & Long term depression \\
\hline LTP & Long term potentiation \\
\hline MAPK & Mitogen-activated protein kinase \\
\hline MDB & Methyl-CpG binding domain \\
\hline
\end{tabular}




\begin{tabular}{|c|c|}
\hline MeCP2 & Methyl-CpG binding protein 2 \\
\hline mIPSC & Miniature inhibitory postsynaptic currents \\
\hline $\mathrm{mLST8}$ & Mammalian lethal with sec-13 protein 8 \\
\hline Moco & Molybdenum cofactor \\
\hline mSin1 & Mammalian stress-activated Map kinase-interacting protein 1 \\
\hline mTOR & Mechanistic target of rapamycin \\
\hline mTORC $1 / 2$ & mTOR complex $1 / 2$ \\
\hline NL1-4 & Neuroligin isoform $1-4$ \\
\hline PDE4D & Phosphodiesterase 4D \\
\hline $\mathrm{PH}$ & Pleckstrin homology \\
\hline PI3K & Phosphatidylinositol 3 kinase \\
\hline PKA & Protein kinase $\mathrm{A}$ \\
\hline PP1 & Protein phosphatase 1 \\
\hline PP2A & Protein Phosphatase $2 \mathrm{~A}$ \\
\hline PPAR-Y & Peroxisome proliferator-activated receptor $\mathrm{Y}$ \\
\hline PPlases & Peptidyl-prolyl cis-trans isomerases \\
\hline PRAS40 & Proline-rich Akt substrate of $40 \mathrm{kDa}$ \\
\hline Protor & Protein observed with Rictor $1 / 2$ \\
\hline ps & pico second \\
\hline PTEN & Phosphatase and tensin homolog \\
\hline $\mathrm{R}$ & Arginine \\
\hline Raptor & Regulatory associated protein of mTOR \\
\hline REF-52 & Rat embryonic fibroblasts-52 \\
\hline Rheb & Ras homolog enriched in the brain \\
\hline Rictor & Rapamycin-insensitive companion of mTOR \\
\hline RLD & Rapid lifetime determination \\
\hline RSK & Ribosomal S6 kinase \\
\hline
\end{tabular}




$\begin{array}{ll}\text { RTT } & \text { Rett syndrome } \\ R_{0} & \text { Förster distance } \\ \text { S } & \text { Serine } \\ \text { SGK-1 } & \text { Serum-glucocorticoid-regulated kinase-1 } \\ \text { SH3- } & \text { Src-homology domain } \\ \text { SKAR } & \text { S6K1/Aly/REF } \\ \text { SREBP1/2 } & \text { Sterol regulatory element-binding protein1/2 } \\ \text { S6K1/2 } & \text { S6 kinase 1/2 } \\ \text { T } & \text { Threonine } \\ \text { TCSPC } & \text { Time correlated single photon counting } \\ \text { TD } & \text { Time domain } \\ \text { TrkB } & \text { tropomyosin related kinase B } \\ \text { TSC1 } & \text { Hamartin } \\ \text { TSC2 } & \text { Tuberin } \\ \text { VHH } & \text { Variable heavy chain } \\ \text { WT } & \text { wildtype } \\ 4 E-B P 1 / 2 / 3 & 4 E \text { binding protein1/2/3 } \\ & \end{array}$




\section{List of Figures}

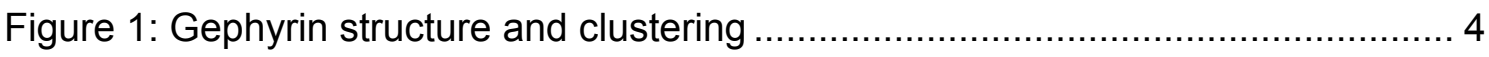

Figure 2: Organisation of the inhibitory postsynapse .................................... 11

Figure 3: Activators and effectors of mTORC1 and mTORC2 ........................... 22

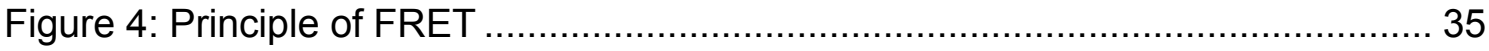

Figure 5: Principle of time correlated single photon counting FLIM ........................ 37

Figure 6: Antibody staining of total and phosphorylated mTOR ......................... 53

Figure 7: Schematic for staining procedure without blocking antibodies ................. 55

Figure 8: Staining of mTOR phosphorylation without blocking antibodies............... 57

Figure 9: Schematic for staining procedure with blocking antibodies ..................... 58

Figure 10: mTOR phopsphorylation assay with blocking antibodies ......................59

Figure 11: Exchanging Cy3 for ATTO 532 is beneficial for FRET ....................... 61

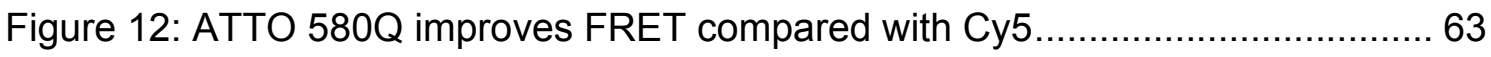

Figure 13: Stimulation and inhibition of mTOR phosphorylation ........................... 64

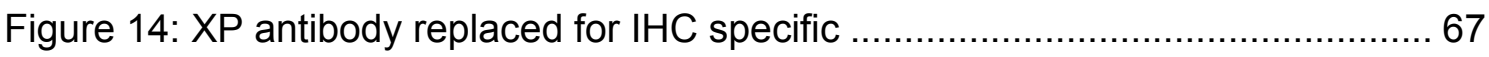

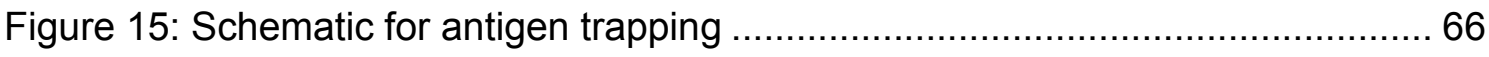

Figure 16: Comparison between the $7 \mathrm{C} 10$ and abcam antibody ............................ 70

Figure 17: Direct comparison between the XP and IHC-specific antibody ................72

Figure 18: Reduced mTOR phosphorylation in MeCP2-KO neurons ...................... 74

Figure 19: Akt phosphorylation assay with inverted response ............................ 77

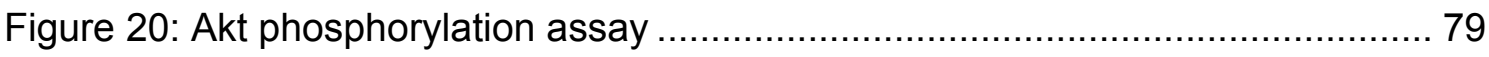

Figure 21: Chronic rapamycin treatment increases Akt phosphorylation at $S 473 \ldots . .81$

Figure 22: Reduced Akt phosphorylation in MeCP2-KO neurons......................... 83

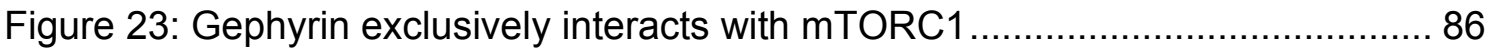

Figure 24:mTOR gephyrin interactions is dependent on gephyrin pS270 .............. 88 
Figure 25: Gephyrin trimerisation is inhibited by mTORC1 in HEK293 cells.....

Figure 26: Gephyrin mutants reduce clustering

92

Figure 27: Gephyrin trimerisation promoted by collybistin 2 (-SH3) and neuroligin 293

Figure 28:Gephyrin dimerization promoted by collybistin 2 (-SH3) and neuroligin 2. 95

Figure 29: Gephyrin trimerisation at GABAergic synapses is inhibited by mTORC1 98

\section{List of Tables}

Table 1: Genotyping primers................................................................... 44

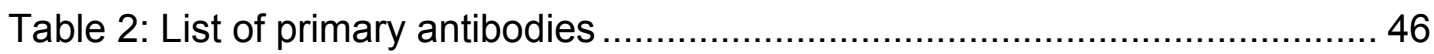

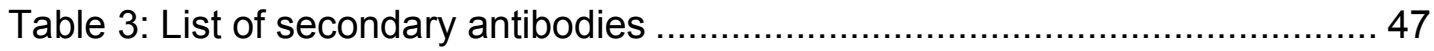

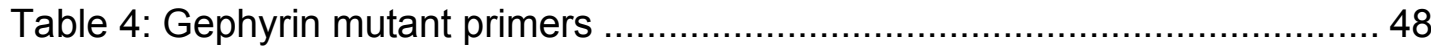

Table 5: C-terminally labelled Gephyrin constructs ...................................... 49 


\section{Summary}

The mechanistic target of rapamycin (mTOR) perceives and integrates various extracellular and intracellular signals and regulates cellular growth and homeostasis. The evolutionary conserved serine/threonine kinase can form two functionally and structurally distinct complexes, mTORC1 and mTORC2. Regulation of these complexes occurs through levels of glucose and amino acids, energy levels, hormones, growth factors and neurotransmitters. mTOR regulates mRNA translation, lipid biosynthesis, auto- and mitophagy, and lysosome biogenesis. Signalling pathways that are regulated by $\mathrm{mTOR}$ are involved in integrated processes of neuronal development, synapse formation and plasticity, memory and cognition. Brain-related alterations in mTOR signalling are connected to pathological conditions like epilepsy, depression, autism, schizophrenia, Alzheimer's and Parkinson's disease.

While some aspects of mTOR signalling, like the regulation of mRNA translation, are well understood, others remain loosely defined. We have therefore developed two quantitative optical tools that analyse the endogenous activity of mTORC1 and mTORC2. We use antibodies in ratiometric FRET assays to quantify activity states of both mTOR complexes. The application of these assays to neurons from MeCP2-KO mice, a mouse model of Rett syndrome, a severe neurodevelopmental disorder, confirmed a reduced mTORC1 activity in MeCP2-KO neurons. These experiments also suggested a neuron-specific reduction of mTORC1 activity as well as a general reduction in $\mathrm{mTORC2}$ signalling. These impairments may represent a reason for the observed reduced number of synapses in Rett syndrome.

The interaction of mTOR with gephyrin, the major organiser of the inhibitory postsynapse, has been described in the past. This interaction has the potential to regulate inhibitory synapse formation and plasticity by localised protein translation at the inhibitory postsynapse. We developed a FRET assay that confirmed the interaction between mTOR and gephyrin. We found that gephyrin exclusively interacted with mTORC1, and that serine 270 of gephyrin is an important determinant of this interaction. Serine 270 is a target of GSK-3 $\beta$ and CDK5 in the brain, which seem to play opposing roles in the regulation of synapse formation. The GSK- 
$3 \beta / C D K 5$ mediated recruitment of $\mathrm{mTORC} 1$ to the postsynaptic gephyrin network may represent a mechanism for regulating synapse formation and plasticity.

In order to organise the inhibitory postsynapse, gephyrin forms a 2-dimensional network underneath the synaptic membrane. Synaptic adhesion molecules and inhibitory neurotransmitter receptors interact with the gephyrin network and are organised by it. We developed two FRET assays that measure the formation of the gephyrin network. We demonstrate that inhibition of mTORC1 promotes the formation of the gephyrin network at GABAergic postsynaptic sites. This may represent a regulatory mechanism for inhibitory synapse formation and plasticity. 


\section{Zusammenfassung}

Das Protein mechanistic target of rapamycin (mTOR) empfängt und integriert vielfältige extrazelluläre sowie intrazelluläre Signale und reguliert zelluläres Wachstum und Homöostase. Es handelt sich um eine evolutionär konservierte Serin/Threonin Kinase, die zwei strukturell und funktionell unterschiedliche Komplexe bildet, mTORC1 und mTORC2. Die Regulation dieser Komplexe erfolgt durch Nährstoffe, wie Glukose und Aminosäuren, den Energiegehalt der Zelle, Hormone, Wachstumsfaktoren sowie Neurotransmitter. mTOR reguliert die mRNS-Translation, die Lipidbiosynthese, die Auto-/Mitophagie und die Lysosombildung. Signalwege die durch mTOR reguliert werden umfassen integrierte Prozesse wie die neuronale Entwicklung, Synapsenbildung und -plastizität, Erinnerung und Wahrnehmung. Veränderungen der mTOR Signalgebung im Gehirn stehen mit pathologischen Zuständen wie Epilepsie, Depressionen, Autismus, Schizzophrenie, Alzheimer und der Parkinson Krankheit in Verbindung.

Während einige Aspekte der mTOR Signalgebung gut bestimmt sind, sind andere kaum definiert. Wir haben deshalb quantitative optische Werkzeuge entwickelt die die Analyse der mTORC1- sowie mTORC2-Aktivität erlauben. Wir benutzen Antikörper in ratiometrischen FRET-Analyse0 um die Aktivitätszustände von mTOR zu messen. Die Anwendung dieser Analysen in Neuronen aus MeCP2 knockout Mäusen, einem Mausmodell des Rett-Syndroms, konnten bestätigen, das die mTORAktivität in den Gehirnen dieser Mäuse reduziert war. Diese Experimente deuteten außerdem eine neuron-spezifische Reduzierung der mTORC1-Aktivität, sowie eine allgemeine Verringerung der mTORC2-Aktivität an. Diese Veränderungen könnten einen Grund für die beobachtete reduzierte Anzahl von Synapsen bei Rett Mäusen darstellen.

Die Interaktion von mTOR und Gephyrin, dem wichtigsten Organisator der inhibierenden Postsynapse, wurde bereits in der Vergangenheit beschrieben. Diese Interaktion hat das Potenzial, die Bildung und Plastizität inhibitorischer Synapsen durch lokale Proteintranslation zu bestimmen. Wir entwickelten eine FRET Analyse welche die Interaktion zwischen mTOR und Gephyrin auf Einzelzellniveau zeigt und quantifiziert. Wir konnten zeigen, das Gephyrin exklusiv mit mTORC1 interagiert und das Serin 270 in Gephyrin eine wichtige Rolle für die Interaktion mit Gephyrin spielt. 
Um die hemmende Postsynapse zu organisieren, bildet Gephyrin ein zweidimensionales Netzwerk unter der postsynaptischen Membran. Synaptische Adhäsionsmoleküle und hemmende Neurotransmitter-Rezeptoren interagieren mit diesem Netzwerk. Wir entwickelten zwei FRET-Analysen, welche die Bildung des Netzwerkes quantitativ bestimmen können. Damit konnten wir zeigen, das die Vernetzung durch Hemmung von mTORC1 befördert wird. Dies stellt möglicherweise einen neuen Regulationsmechanismus der Synapsenbildung und Plastizität dar. 


\section{Introduction}

\subsection{Inhibitory neurotransmission}

Neuronal inhibition has two major functions: interrupting activity and the restricting activation patterns in time and space, providing the necessary autonomy for neighbouring excitatory cells and guaranteeing their functional diversity. For this purpose the brain contains multiple classes of specialized interneurons that selectively innervate excitatory neurons and other interneurons by forming inhibitory synapses.

Inhibitory synapses are basic structures required for information-processing within neural circuits. Their spatial arrangement and temporal activation sequence allows for sophisticated analysis of sensory inputs on all organizational levels of the nervous system.

In the central nervous system fast synaptic inhibition is facilitated by two neurotransmitters: GABA and glycine. These are released from presynaptic active zones of inhibitory interneurons onto membrane specialisations of their target cells, the inhibitory postsynapse. Simplified, the inhibitory postsynapse contains neurotransmitter receptors, synaptic adhesion molecules, scaffolding molecules, and accessory signalling molecules that relay inhibitory inputs onto target cells.

Synaptic release of GABA and glycine activates ligand-gated chloride channels, like

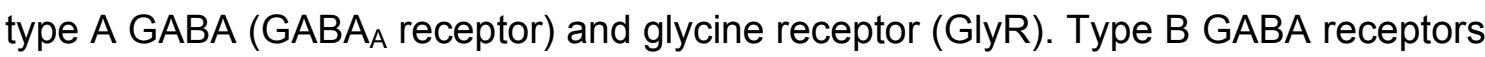
belong to the class of $G$ protein-coupled receptors that activate potassium channels, and mediate slow inhibition. Inhibitory neurotransmitter receptors are anchored and clustered at the postsynaptic specialisation, a scaffolding structure underneath the postsynaptic membrane which contains the scaffolding molecule Gephyrin. Gephyrin also interacts with multiple classes of signalling molecules and undergoes complex post-translational modifications, making it a major regulator of synaptic inhibition in the central nervous system. 


\subsection{Gephyrin}

\subsubsection{Structure and function}

Gephyrin is a highly conserved protein that is widely expressed in vertebrate tissues where it plays a multifunctional role (Ogino et al. 2011; Nawrotzki et al. 2012). In nonneuronal cells, gephyrin's primary function is the synthesis of molybdenum cofactor (Moco), a co-enzyme involved in catalysing redox reactions (Stallmeyer et al. 1999; Schwarz et al. 2009). It's other function is specific to neurons as it structurally and functionally organizes the inhibitory post-synapse (Fritschy et al. 2008; Tretter et al. 2012). Gephyrin was first isolated from glycine receptor preparations, where it was also bound to polymerized tubulin (Pfeiffer et al. 1982). Supposedly its function was to bridge neurotransmitter receptors and the cytoskeleton (Kirsch et al. 1991).

Today it is widely accepted that gephyrin's role at the inhibitory postsynapse is not restricted to this structural function as a scaffolding protein. Gephyrin also represents as a major signalling hub for various inputs involved in synapse formation, modulation, synaptic plasticity, and transport processes, making it a potential master regulator of the inhibitory synaptic transmission (Tyagarajan and Fritschy 2014).

Gephyrin is a $93 \mathrm{kDa}$ protein comprising of 3 domains, an N-terminal G-domain followed by a flexible linker domain also referred to as the C-domain, and an Edomain at the C-terminus. Sequence analysis revealed a high degree of homology with two bacterial enzymes, MogA and MoeA, both involved in consecutive steps of aforementioned molybdenum cofactor biosynthesis. The gephyrin gene seems to have evolved by fusion of the MogA and the MoeA genes (Fritschy et al. 2008). In mammals gephyrin is highly abundant in liver, kidney and other non-neuronal tissues, where it is believed to catalyse molybdenum cofactor synthesis independent of its aggregation state and neuronal function (Schwarz et al. 2009). In the nervous system molybdenum cofactor synthesis seems to be restricted to astrocytes and the significance of Moco synthesis for synaptic function yet to be demonstrated (Smolinsky et al. 2008).

Dual-label electron microscopy and fluorescence microscopy revealed that gephyrin immunoreactive punctae, termed "clusters", were found to be between 50 and $200 \mathrm{~nm}^{2}$ in size. Inside these clusters, gephyrin exists in a self-aggregated state at 
very high local concentrations (Triller et al. 1985; Sassoe-Pognetto et al. 1995; Sassoè-Pognetto et al. 2000, Lardi-Studler et al. 2007).

Analysis of the self-aggregation properties of gephyrin showed that isolated Gdomains spontaneously form trimers while isolated E-domains form dimers (Schwarz et al. 2001; Sola et al. 2004). The binding site for the glycine receptor has been mapped to the E-domain (Schrader et al. 2004; Kim et al. 2006). These observations integrate into a model in which three gephyrin molecules form a star-shaped trimer via their G-domains. The 3 outwardly oriented E-domains of a gephyrin trimer molecule may recruit other gephyrin trimers building up a hexagonal lattice structure that binds to glycine- or $\mathrm{GABA}_{\mathrm{A}}$ receptors (Figure 1A). A recent report points out that the $\mathrm{C}$-domain may play an important role in regulating gephyrin clustering. Because of its unstructured folding as well as interactions with the $G$ and the $E$ domain, gephyrin may be able to occupy different states of extension which could provide structural flexibility within the scaffold (Sander et al. 2013) (Figure 1B).

The so called lattice model of gephyrin clustering is based purely on observations made with single isolated protein domains expressed in recombinant expression systems in which no mammalian post-translational modifications are implemented. In vitro gephyrin has been shown to undergo extensive post-translational modifications like phosphorylation and acetylation. It is believed that these modifications are significant for synaptic localization and aggregation of gephyrin but may also play a role in synaptic signalling. Accordingly, the lattice model was called into question after it was reported that full-length gephyrin expressed in vertebrate cells shows very different aggregation properties with a stacked double-trimer being the predominant form of gephyrin aggregates. In the alternate stacked trimer model, gephyrin forms trimers via the $\mathrm{G}$ or $\mathrm{G}$ and $\mathrm{C}$ domain and these trimers are then formed into stacks via an E-domain interaction (Figure $1 \mathrm{C}$ and $1 \mathrm{D}$ ).

In both models, the E-domains point outwardly and allow the interaction with glycine receptors, neuroligin 2 (NL2) or collybistin (CB) However, only the hexagonal lattice model is consistent with the observed 1:1 ratio of gephyrin to bound receptors at glycinergic and GABAergic postsynapses (Specht et al. 2013). 


\section{A}

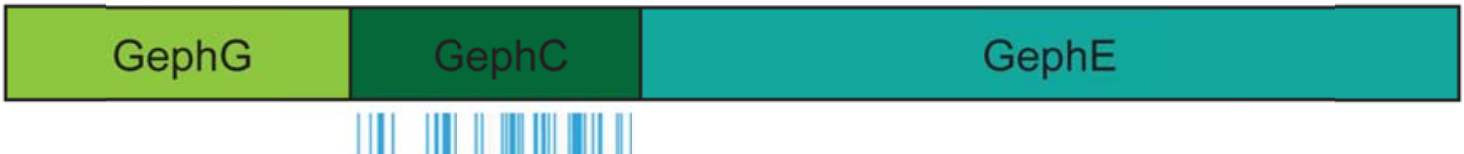

B

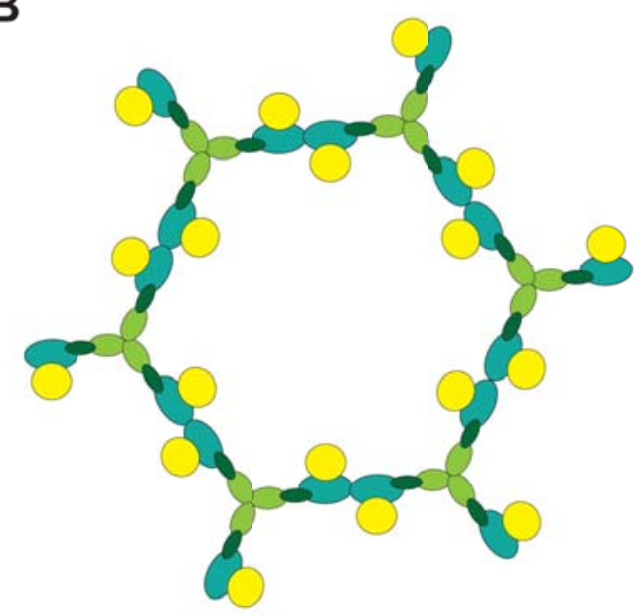

D

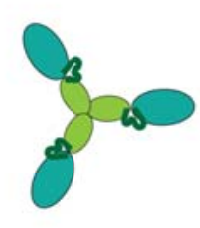

C
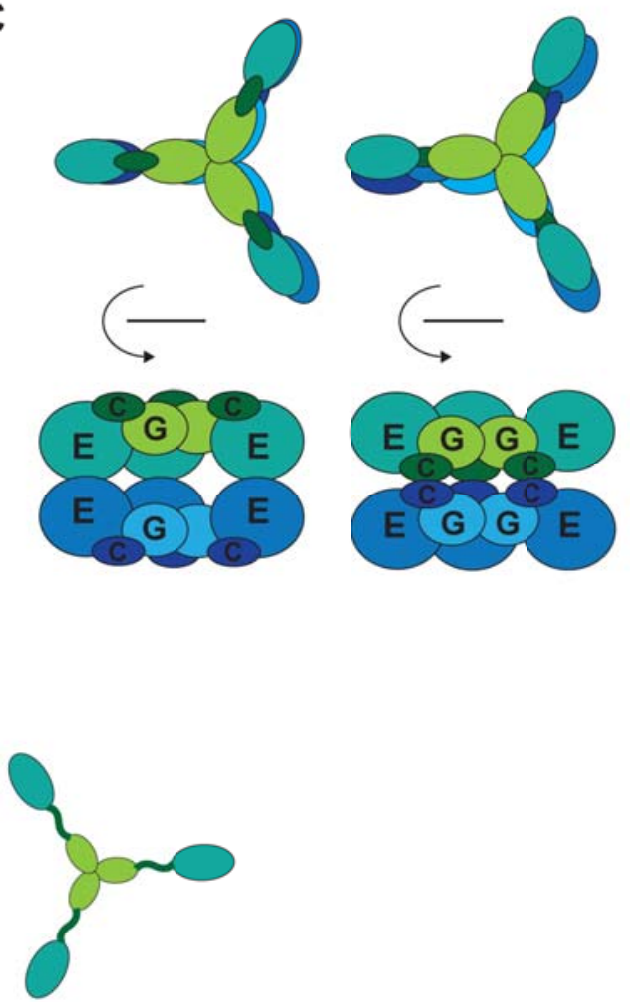

\section{Figure 1: Gephyrin structure and clustering}

A: Rat gephyrin is comprised of three domains, an N-terminal G-domain, GephG (light green), regulatory C-domain, GephC (dark green) and C-terminal E-domain, GephE (mint green). Potential phosphorylation sites are marked below the GephC domain (blue lines). Start and end of domains are numbered above. B: The hexagonal lattice model of gephyrin clustering. Gephyrin forms trimers via the G-domain. Gephyrin trimers then dimerise via the E-domain and form a hexagonal lattice. Glycine receptors bind to the E-domain (yellow circles). C: An alternate oligomerisation model is of stacked gephyrin trimers interacting via their $\mathrm{C}$ - or E-domains. D: A flexible C-domain allows gephyrin trimers to occupy different extension states. 


\subsubsection{Gephyrin and postsynaptic receptors}

Glycine- and $\mathrm{GABA}_{\mathrm{A}}$-receptors are pentameric chloride channels that together with nicotinic acetylcholine receptors and serotonin receptors make up a superfamily of Cys-loop ligand-gated ion channels.

Glycine receptors are comprised of 5 subunits, $\alpha_{1}-\alpha_{4}$ and $\beta$, encoded by 5 genes. These subunits assemble into homomeric (only $\alpha$-subunits) or heteromeric ( $\alpha$ - and $\beta$ subunits) receptors which colocalise perfectly with gephyrin when visualized by immunofluorescence (Triller et al. 1985). The high spatial correlation is caused by the high binding affinity between gephyrin and the intracellular loop of the $\beta$-subunit (Kirsch et al. 1991; Meyer et al. 1995; Dumoulin 2009). This binding affinity is high enough for gephyrin to bind to glycine receptor-containing transport vesicles leading to a co-transport of both molecules within dendrites. Binding of gephyrin to dynein motor proteins forms a GlyR-gephyrin-dynein transport complex. Activity dependend polyglutamyolation of tubulin contributes to the dynamic regulation of the glycinergic postsynapse by the transport rate of GlyR-gephyrin aggregates (Hanus 2004; Maas et al. 2006).

$\mathrm{GABA}_{\mathrm{A}}$-receptors show extensive subunit heterogeneity with 19 genes encoding for subunits $\alpha_{1}-\alpha_{6}, \beta_{1}-\beta_{3}, Y_{1}-Y_{3}, \delta, \varepsilon, \pi, \theta$ and $\rho_{1}-\rho_{3}$. A full receptor pentamer is usually assembled from at least 3 different classes of subunits, for example $\alpha-, \beta$ - and $\gamma$ - or $\alpha-, \beta$ - and $\delta$-subunits (Barnard et al. 1998). Only the subset of $\alpha_{1}, \alpha_{2}$ or $\alpha_{3}$ subunits along with $\gamma_{2}$ subunit combinations colocalises with gephyrin clusters at inhibitory postsynapses (Tretter et al. 2008; Mukherjee et al. 2011; Tretter et al. 2011; Kowalczyk et al. 2013). By contrast $\alpha_{4}$, and $\alpha_{5}$ and $\delta$-subunits containing $G_{A B A_{A^{-}}}$ receptors are mainly localised extrasynapticly and do not colocalise with gephyrin. Targeted deletion of the $\mathrm{Y}_{2}$-subunit reduces the postsynaptic gephyrin cluster formation although $\gamma_{2}$ does not directly interact with gephyrin. This indirect effect may be due to reduced cell surface expression of $\mathrm{V}_{2}$-deficient $\mathrm{GABA}_{\mathrm{A}}$-receptors (Günther et al. 1995).

The role of gephyrin in postsynaptic clustering of $G A B A_{A}$-receptors is not entirely clear. GPHN knockout $(\mathrm{KO})$ mice show impeded but not abolished $\mathrm{GABA}_{\mathrm{A}}$-receptors clustering depending on the neuronal subtype (Fischer et al. 2000; Kneussel et al. 2001; Levi 2004). However, in GABAergic postsynapses, gephyrin clustering depends on the presence of $G_{A B A}$-receptors. More precisely on the presence of 
certain subtypes which have substantial influence on the molecular composition and function of the inhibitory postsynapse (Fritschy 2006; Kralic et al. 2006; Peden et al. 2008).

The structural heterogeneity of $\mathrm{GABA}_{\mathrm{A}}$-receptors seems to provide variability to the molecular and functional properties of GABAergic synapses (Wu et al. 2012). In addition, GABAergic synapses are equipped with distinct signalling complexes that are specific to subcellular compartments or neuronal subtypes. For example the dystrophin-glycoprotein complex (DGC) which regulates postsynaptic anchoring of a selective subset of $\mathrm{GABA}_{A}$-receptors, independently of gephyrin in cortical and cerebellar synapses (Kneussel and Betz 2000).

\subsection{Regulators of gephyrin clustering}

\subsubsection{Neuroligins}

Neuroligins are postsynaptic adhesion molecules that, together with their presynaptic counterparts the neurexins, play a fundamental role in excitatory and inhibitory synapse formation and function. Neuroligins are expressed in 4 isoforms, neuroligin1 to -4 (NL 1-4) (Hu et al. 2015). The presynaptic neurexins bind to all neuroligin isoforms, but, in contrast to neurexins, neuroligins are specifically localized to particular synapses. NL1 is only present in excitatory synapses (Chubykin et al. 2007) while NL2 and 4 are present at glycinergic and GABAergic synapses (Varoqueaux et al. 2004; Hoon et al. 2011). NL3 is present at both types of synapses (Budreck and Scheiffele 2007). NL2 is the only synaptic adhesion molecule known to bind gephyrin in GABAergic synapses. It is therefore believed to facilitate gephyrin and $\mathrm{GABA}_{A}$-receptors clustering in nascent GABAergic postsynapses (Poulopoulos et al. 2009).

The targeted deletion of single NL isoforms was not sufficient to unveil the specific function of NL probably due to compensatory effects of NL1 and NL3 and NL2, NL4 and NL3.

NL2-deficient mice display region-specific alterations of GABAergic synapses. These mice show reduced $G A B A_{A}$-receptor clusters in the retina (Hoon et al. 2009), while in 
the hippocampus and the dentate gyrus, perisomatic but not dendritic $G_{B A A_{A}}$ receptors and gephyrin clusters are lost (Gibson et al. 2009; Jedlicka et al. 2011). The major behavioural aberration observed in these mice is increased anxiety, which is compatible with reduced inhibitory transmission in brain regions that regulate emotional behaviour (Blundell et al. 2009).

\subsubsection{Collybistin}

Collybistin has been found to interact with gephyrin and is able to translocate gephyrin to the plasma membrane in non-neuronal cells (Kins et al. 2000). It is a neuron-specific guanosine triphosphate exchange factor (GEF) for the cell division control protein 42 (CDC42) (Xiang et al. 2006). It belongs to the DBL family of GEFs. All members of this family activate small GTPases of the Rho family and comprise of three domains that align in a fixed order: an N-terminal type 3 Src-homology domain (SH3-), followed by a DBL homology ( $\mathrm{DH}-)$ domain which possesses GEF activity and a C-terminal pleckstrin homology (PH-) domain (Miller et al. 2013).

Collybistin pre-mRNA is coded by the gene Arhgef9 and is subject to extensive alternative splicing, which gives rise to three isoforms (CB1-CB3) that differ in their C-terminus. Further splicing generates 2 versions of each isoform with or without the $\mathrm{N}$-terminal $\mathrm{SH} 3$ domain. The $\mathrm{SH} 3$ domain can bind to the $\mathrm{DH}$ domain rendering collybistin inactive by blocking its catalytic centre. It is assumed that the autoinhibition is released by binding of collybistin to NL2 via the SH3 domain (Soykan et al. 2014).

The functional relevance of the known 6 isoforms of collybistin is not yet determined, but the expression of different isoforms in neurons points toward non-overlapping functions (Chiou et al. 2011; Tyagarajan et al. 2011; Körber et al. 2012; Fritschy et al. 2012).

In non-neuronal cells, only the SH3 domain-deficient splice variant of CB2 is capable to translocate and activate gephyrin at the plasma membrane (Kins et al. 2000). To do so, collybistin binds gephyrin at the C-terminal collybistin binding domain (CBD) at a binding site that is overlapping with the binding site for GABA subunits (Grosskreutz et al., 2001; Harvey et al., 2004). In turn, gephyrin binds to 
collybistin's DH domain which might interfere with collybistin's ability to activate CDC42 (Xiang et al. 2006).

The Arhgef9 null mouse revealed that collybistin is not required for gephyrin clustering or glycine receptor co-clustering at glycinergic synapses. More importantly, this mouse model revealed a remarkable cell- and synapse-specific heterogeneity of gephyrin clustering phenotypes in the GABAergic system. In Purkinje cells, gephyrinbut not $\mathrm{GABA}_{\mathrm{A}}$-receptor clustering was disrupted while in hippocampal and cortical pyramidal cells gephyrin and $\mathrm{GABA}_{\mathrm{A}}$-receptor clustering was impaired and GABAergic transmission was reduced (Papadopoulos et al. 2008). Behaviourally, the Arhgef9 null mice showed increased signs of anxiety and impaired spatial learning compatible with the reduced inhibitory neurotransmission phenotype. In agreement with this mouse model, a human case of Arhgef9 gene deletion was reported to show a severely deficient adaptive behaviour, moderate to mild autism and frequent epileptic discharges (Machado et al. 2015).

\subsection{Gephyrin clustering}

\subsubsection{The receptor activation model}

The receptor activation model aims to explain the role of gephyrin in glycine- and $\mathrm{GABA}_{\mathrm{A}}$-receptor clustering at postsynaptic sites. In immature neurons, an initial release of presynaptic GABA or glycine induces membrane depolarisation due to chloride efflux. In immature neurons the chloride concentration is higher inside than outside so that a change in chloride permeability leads to a depolarisation instead of a further polarisation like in mature neurons (Ben-Ari et al., 1989). This depolarisation leads to calcium influx through voltage-dependent calcium channels which induces the membrane apposition of GlyR and hence the clustering of gephyrin (Kneussel and Betz 2000). Clustered gephyrin binds via adaptor molecules to the actin cytoskeleton and to polymerised tubulin and starts to immobilise more glycine and GABA receptors. Furthermore calcium influx induces the release of brain derived neurotrophic factor (BDNF) which activates phosphatidylinositol 3 kinase (PI3K) which promotes the production of phosphatidylinositol-3,4,5-triphosphate. The latter recruits collybistin to further promote gephyrin clustering. 


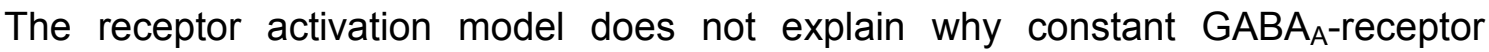
antagonism does not prevent the formation of GABAergic synapses in vitro. Therefore the key role for activating gephyrin clustering was attributed to collybistin rather than receptor activation. Until today no other model has replaced the receptor activation model and it is not known which synaptic adhesion molecule triggers gephyrin clustering. Nevertheless, the ability of gephyrin to bind transsynaptic adhesion molecules in combination with their synapse-specific segregation raises the question if gephyrin is involved in determining neurotransmitter specificity by assembling a certain type of receptors.

\subsubsection{Gephyrin clustering at glycinergic synapses}

The Arhgef9 knock out mouse demonstrated that collybistin is dispensable for gephyrin clustering at glycinergic synapses as these mice do not exhibit glycinergic synapse dysfunction and are viable and fertile (Papadopoulos et al. 2008).

It is therefore assumed that the observed co-transport of gephyrin on glycinereceptor carrying transport vesicles is the foundation of gephyrin clustering in glycinergic synapses. After gephyrin-decorated glycine receptors appear on the cell surface, they diffuse laterally within the plasma membrane until they reach a synaptic spot where gephyrin starts to cluster upon binding to synaptic adhesion molecules (Dumoulin 2009; Calamai et al. 2009). The clustering and accumulation is regulated by several factors. For example integrin $\beta 1$ and $\beta 3$ have been shown to regulate the postsynaptic accumulation of glycine receptors and the binding to the gephyrin scaffold (Charrier et al. 2006). Protein kinase C was reported to influence receptor diffusion and gephyrin interaction (Specht et al. 2013). And ultimately, heat shock cognate protein of $70 \mathrm{kDa}$ can regulate gephyrin clustering without influencing glycine receptor accumulation (Machado et al. 2011).

\subsubsection{Gephyrin clustering at GABAergic synapses}

A model for gephyrin clustering at GABAergic synapses is based on the observation that neuroligin 2 is able to interact with collybistin and gephyrin. It is thought that 
binding to neuroligin 2 activates collybistin which in turn recruits gephyrin to the plasma membrane. Clustered gephyrin then immobilizes $\mathrm{GABA}_{\mathrm{A}}$-receptors at nascent GABAergic synapses.

An alternative model postulates that, first, a trimeric complex of GABA $A_{A}$-receptor $\alpha_{2}-$ subunit, collybistin and gephyrin forms which disinhibits collybistin and nucleates the formation of inhibitory postsynapses. Both models require collybistin activation as a prerequisite for gephyrin clustering. Consequently, both models fail to explain the heterogeneity of gephyrin clustering phenotypes in the Arhgef9 null mouse. It will therefore be necessary to take the molecular heterogeneity of receptors, synapses and regions into account in order to understand the complexity of gephyrin clustering at GABAergic synapses (Figure 2). 


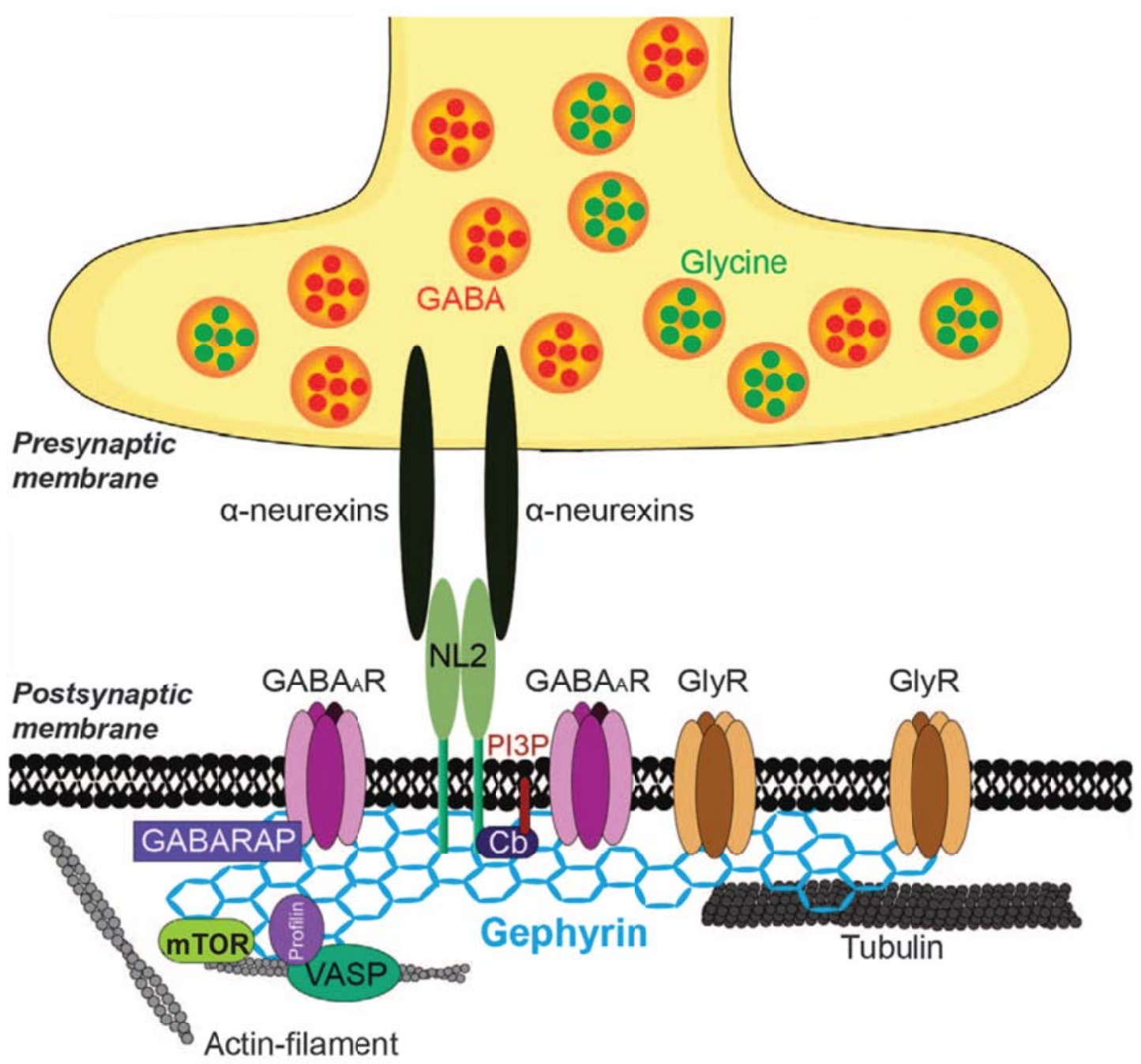

Figure 2: Organisation of the inhibitory postsynapse

The coordination of the pre- and postsynaptic terminal is mediated by transsynaptic interaction of $\alpha$-neurexins with neuroligin 2 (NL2) through the synaptic cleft. On the postsynaptic side, NL2 and collybistin $(\mathrm{Cb})$ recruit gephyrin (light blue) to the postsynaptic membrane, where gephyrin is thought cluster into a hexagonal lattice. Gephyrin organises the postsynaptic terminal by binding to glycine- and GABAA receptors. Gephyrin interacts with the cytoskeleton by binding to tubulin and actin filament adaptor protein profiling and VASP. Gephyrin represents the major organizer of the inhibitory postsynapse and is thought to have a signalling function. Interactions with signalling molecules are thought to play a role in inhibitory synaptic plasticity. Modified from Choi and Ko, 2014. 


\subsubsection{Gephyrin phosphorylation in GABAergic synapse plasticity}

\subsubsection{GSK-3 $\beta$ and CDK5 dependent phosphorylation of serine 270}

Gephyrin has been identified as a highly phosphorylated protein very early on (Langosh et al., 1992), but the functional relevance of the phosphorylation has long been neglected for its mere structural role. Gephyrin harbours 22 serine- $(S)$ and threonine- (T) phosphorylation motives in its regulatory C-domain (Sander et al. 2013). It also represents the most flexible and accessible part in the molecule and is therefore the most likely target for posttranslational modifications (Herweg and Schwarz 2012). Phosphorylation could lead to conformational changes of the Cdomain relative to the adjacent G- and E-domains, and alter the clustering properties of the molecule. An interaction of the $\mathrm{C}$-domain with the $\mathrm{E}$-domain as well as the $\mathrm{G}$ domain was reported to regulate different states of extension of gephyrin which represents a potential mechanism of regulating gephyrin clustering (Sander et al. 2013).

The first identified phosphorylation site that is targeted by a specific kinase was S270, phosphorylated by glycogen synthase kinase $3 \beta$ (GSK-3 $\beta$ ) (Tyagarajan et al. 2011). In the brain GSK-3 $\beta$ is involved in developmental processes like neurogenesis, polarization and axon outgrowth (Hur and Zhou 2010). Alanine (A) exchange mutations of $S 270$ produces a phenotype with supernumerary gephyrin clusters and GABAergic synapses which leads to enhanced frequencies of miniature inhibitory post-synaptic currents (mIPSC). Pharmacological blockage of GSK-3 $\beta$ activity using $\mathrm{LiCl}$ shows a similar effect. In the brain, protein kinase Akt inhibits GSK$3 \beta$ which potentially connects neuronal activity by calcium-induced activation of the BDNF-TrkB-Akt signalling pathway to posttranslational modfication of gephyrin at S270. The reduced phosphorylation seems to stimulate the formation of new inhibitory synapses. Alanine exchange mutations as well as inhibition of GSK-3 $\beta$, recapitulates this effect.

In excitatory synapses, the link between synaptic activity and protein translation is well established. It is a process involved in long term plasticity that connects NMDAreceptor activity and calcium influx to BDNF autocrine activation of the Akt/mTOR/S6 axis that induces the local translation of synaptic proteins (Hoeffer and Klann 2010). 
A potential mechanism for inhibitory synapses has been suggested by Sabatini and colleagues (1999). They describe the interaction between mechanistic target of rapamycin (mTOR), the major regulator of cap-dependent protein translation, and gephyrin. The authors also demonstrate that mutants of mTOR that were unable to bind to gephyrin also fail to activate downstream targets responsible for mRNA translation initiation (Sabatini et al. 1999). The link between mTOR and gephyrin still lacks confirmation in inhibitory synapses and it would be interesting if this indicated connection could be further substantiated.

A second serine-threonine kinase that is capable of phosphorylating gephyrin at S270 is cyclin-dependent kinase 5 (CDK5). CDK5 is implicated in several aspects of neurodevelopment and neuronal function like neuronal migration, differentiation, synaptic function, homeostasis and plasticity (Su and Tsai 2011). In excitatory synapses, CDK5 regulates synaptic plasticity by 3 different mechanisms, 1: it mediates changes in NMDA receptor conductance by phosphorylation of receptor subunits ( $\mathrm{Li}$ et al. 2001); 2: it can reduce the number of NMDA receptors by calpaindependent proteolysis (Hawasli et al. 2007) and 3: it can activate NMDA receptor internalization by phosphorylating PSD-95, inducing LTD (Morabito 2004). In inhibitory synapses CDK5 seems to cooperate with collybistin. Collybistin is known to regulate gephyrin clustering which suggests that phosphorylation of S270 depends on clustered gephyrin (Kuhse et al. 2012).

\subsubsection{ERK1/2 dependent phosphorylation of serine 268}

The mitogen-activated protein kinase (MAPK) cascade that activates extracellular signal-regulated protein kinases-1 and -2 (ERK1 and ERK2) plays a key role in the control of synaptic plasticity in the adult brain. It seems to be essential for neuronal transcriptional events and might regulate synaptic targets directly to control plasticity. The classical way of ERK activation is via receptor tyrosine kinases, adaptor protein binding followed by alterations in the balance between the activities of Ras-GEFs and Ras-GAPs. In neurons, this pathway operates in response to neurotrophic factors. However, ERK activation by membrane depolarization or glutamatergic signalling requires calcium influx facilitated by NMDA receptors or voltage-gated calcium channels. In turn, ERK activity promotes NMDA-dependent and independent forms of 
LTP. It enhances functional properties of glutamate receptors by affecting the trafficking, structural remodelling of activated spines and local protein translation (Thomas and Huganir 2004).

In GABAergic synapses, activated ERK $1 / 2$ phosphorylates gephyrin at S268. Phosphorylation of S268 seems to limit the size of gephyrin clusters and reduces the amplitude and frequency of mIPSCs in vitro (Tyagarajan et al. 2013). Moreover, phosphorylation of S268 seems to function in synergy with phosphorylation at the neighbouring $\mathbf{S} 270$ to reduce size and number of inhibitory synapses.

\subsubsection{Peptidyl-prolyl cis-trans isomerase Pin-1}

Protein phosphorylations at serine and threonine residues that are followed by a proline residue are called proline-directed phosphorylations. They often lead to conformational changes that are not solely caused the phosphorylation event itself. Instead, they require mediation by chaperone molecules called peptidyl-prolyl cistrans isomerases (PPlases). Pin1 and its homologs are the only known isomerases being able to catalyse isomerisation of already phosphorylated amino acid side chains (Yaffe 1997). These proteins are able to catalyse cis to trans or trans to cis isomerisations in order to create targeting sites for conformation selective prodirected kinases and phosphatases (Weiwad et al. 2000; Zhou 2000). Neuronal targets of Pin-1 are tau, amyloid- $\beta$ protein precursor (APP), $\alpha$-synuclein and neurofilaments which have implications in amyotrophic lateral sclerosis, Parkinson's and Alzheimer's disease (Rudrabhatla and Pant 2010; Lee 2015). Beyond these implications, Pin-1 is involved in apoptosis required for neurodevelopmental processes and in forms of long-term synaptic plasticity at excitatory synapses (Becker and Bonni 2006; Westmark et al. 2010).

In gephyrin, three serine residues have been identified to recruit Pin-1: S188, S194 and S200. Pin1-driven conformational changes have been shown to affect the affinity of GlyR $\beta$-subunit to gephyrin (Moretto Zita et al. 2007). Hippocampal neurons derived from Pin-1 KO mice show less glycine receptor immunoreactive punctae as well as reduced amplitudes of glycine-evoked currents. Hence, Pin-1-dependent conformational changes following phosphorylation events represent a potential mechanism for gephyrin remodelling and synaptic plasticity in glycinergic synapses. 
Pin-1 activity in GABAergic synapses is less well defined but seems to be more orchestrated, with several factors determining gephyrin clustering. The gephyrin recruiting molecules neuroligin 2 and collybistin possess consensus motifs for Pin-1 binding. Gephyrin itself interacts with neuroligin 2 as well as with collybistin via its Edomain. This interaction interface contains a Pin1 consensus sequence located within the neuroligin 2 binding site as well as directly adjacent to the collybistin binding domain (Harvey 2004). The consensus sequence contains a serine (S319) that has been reported to be phosphorylated in vivo (Tyagarajan et al. 2013). Pin1 is therefore competent to modulate the interaction of gephyrin with both of its major modulators. Moreover, the collybistin binding domain overlaps with the interaction site of $\mathrm{GABA}_{\mathrm{A}}$-receptor subunits. Pin-1 dependent conformational changes at this site could therefore affect the recruitment of gephyrin by collybistin and neuroligin 2 as well as the subsequent immobilization of GABA receptors. This conformational switch has therefore the potential to tune GABA transmission subsequent to phosphorylation events exerted on gephyrin.

\subsubsection{Other post-translational modifications of gephyrin}

In a mass spectroscopic analysis of rat gephyrin, 9 acetylated sites were found. Unexpectedly most of the acetylated sites were not prototypical lysine residues but serines or threonines and even more unexpectedly three of these sites were either phosphorylated or acetylated (Tyagarajan et al. 2013). The functional relevance of these acetylations is yet to be determined. It was speculated that acetylations and phosphorylations at phospho-/acetyl-sites may happen contrary to each other in order to prevent the one or the other. For instance, S268 was found to be acetylated in this study which suggests that protein acetylation might add another regulatory step to prevent phosphorylation at this site by ERK1/2 and subsequent downregulation of GABAergic transmission.

The dynamic regulation of the number of $\mathrm{GABA}_{\mathrm{A}}$-receptors provides a key mechanism for functional plasticity of inhibitory synapses (Luscher et al. 2011). Reversible palmitoylation has emerged as the most frequent lipid modification of synaptic proteins with diverse effects on protein trafficking, neuronal development and synaptic plasticity (Fukata and Fukata 2010). In a palmitoyl-proteomic approach 
to identify neuronal palmitoylated proteins, gephyrin was identified as a candidate (Kang et al. 2008). It was confirmed later that gephyrin is palmitoylated at cysteine 212 and 284 by palmitoyl acyltransferase DHHC-12 and that this modification represents an important mechanism to strengthen GABAergic transmission regulated by $\mathrm{GABA}_{\mathrm{A}}$-receptor activity (Dejanovic et al. 2014).

Gephyrin has also been reported to be a target of sumoylation. Sumoylation occurs at lysine-residues and is able to exert various effects including modifications of protein-protein interactions, scaffolding functions or neurotransmitter trafficking and function. Overexpression of sumoylating or de-sumoylating enzymes have shown to influence gephyrin cluster size (Tyagarajan et al. 2012).

Neuronal activity-induced proteolytic cleavage by calpain negatively regulates gephyrin cluster size. Pre-requisite for this regulation seems to be phosphorylation at S268 or S270 by ERK or GSK-3ß. Calpain could exert an additional effect on gephyrin clustering by producing single $G$ and $E$ domains that alter gephyrin clustering properties.

\subsection{Mechanistic target of rapamycin}

mTOR is an evolutionary conserved serine/threonine protein kinase that forms two functionally distinct signalling complexes that are regulated by numerous factors like amino acids -, glucose -, and ATP/ADP levels, growth factors, neurotransmitters or hormones (Figure 3 ). The two mTOR signalling complexes, mTOR complex 1 (mTORC1) and mTOR complex 2 (mTORC2) regulate metabolic processes like capdependent protein translation, energy metabolism, lipid synthesis, cell size, autophagy, mitochondria and lysosome biogenesis (Laplante and Sabatini 2012). In the nervous system, mTOR plays a key role in integrated physiological processes like neuronal development, brain plasticity, learning, memory storage, and cognition. Aberrant mTOR signalling contributes to many pathological conditions ranging from cancer to psychiatric disorders (Bockaert and Marin 2015). The medical application of mTORC1 inhibitors has shown to be beneficial in the treatment of tuberous sclerosis, epilepsy, and different forms of cancers, while activation of mTORC1 signalling has been shown to ease the symptoms of clinical depression (CostaMattioli and Monteggia 2013). 
Rapamycin is a natural macrolide antibiotic produced by the bacterium Streptomyces hygroscopius that was first isolated in the 1970s from soil samples collected at the easter islands, also known as Rapa Nui. It was later described to have immunosuppressant effects and to reduce eukaryotic cell proliferation (Chang and Sehgal 1991). In a genetic study in yeast, it was shown that mutations in three genes Fpr1, Tor1 and Tor2 rendered cells insensitive to the toxic effects of rapamycin (Cafferkey et al. 1993; Helliwell et al. 1994; Chen et al. 1995). This was the foundation for the revelation of one of the most important signalling pathways in biology: the mTOR pathway.

mTOR is expressed in every cell type and regulates a number of fundamental cellular processes. In the brain, mTOR also affects specific neuronal functions like axonal outgrowth (Abe et al. 2010), axon regeneration (Liu et al. 2010), myelination (Bercury et al. 2014; Wahl et al. 2014; Lebrun-Julien et al. 2014), neurotransmitter receptor expression (Duman et al. 2012) or dendritic spine growth (Tavazoie et al. 2005; Tang et al. 2014). Deregulation of mTOR signalling in the brain has been associated with psychiatric conditions like clinical depression or schizophrenia but also severe neurodevelopmental disorders like fragile $X$ syndrome, Down syndrome, autism spectrum disorders, mental retardation, tuberous sclerosis, neurofibromatosis or Rett syndrome (RTT).

Many of the syndromes listed above are caused by or involve upregulated mTORC1 signalling. Some symptoms of these pathologies could be reversed by mTOR inhibition in the corresponding animal models, raising options for treatments in humans.

\subsubsection{Structure function regulation of the mTOR complexes}

The amino acid sequence of mTOR comprises of 2549 residues. A stretch of 20 tandem HEAT repeats (short for Huntington, EF3, A subunit of PP2A, TOR1) is located at the $\mathrm{N}$-terminus. In crystal structures this region is split up into two lobes that are known sites for protein-protein interactions. This segment is followed by a FAT (FRAP, ATM, TRAP) domain and the $\mathrm{N}$ - and the C-lobe of the kinase domain. The C-lobe contains the FRB site (for FRBP12-rapamycin-binding domain) while the C-lobe contains the binding site for mLST8 (mammalian lethal with sec-13 protein 8 ) 
a constitutive member of both mTOR complexes (Yang et al., 2013). Another FAT domain termed FATC is located at the very C-terminus and seems to be necessary for mTOR activity (Hay 2004).

mTOR associates with different proteins to form two functionally distinct complexes: mTORC1 and mTORC2. Both complexes also share some of their components like the already mentioned mLST8. Another one is DEPTOR (DEP domain-containing mTOR-interacting protein) a protein that inhibits mTORC1 and mTORC2 upon overexpression. DEPTOR binds to the FATC domain of mTOR. Another common element of mTORC1 and 2 is the Tti1/Tel2 complex which binds to the second portion of HEAT repeats and is critical for TORC assembly (Laplante and Sabatini 2012).

mTORC1 specific subunits are Raptor (regulatory associated protein of mTOR) and PRAS40 (proline-rich Akt substrate of $40 \mathrm{kDa}$ ). Raptor is an activator of mTOR and binds to the N-terminal HEAT repeats and to the FATC domain while PRAS40 binds to and thereby inhibits Raptor. mTORC1 is sensitive to nutrients like amino acids and glucose, energy levels, some growth factors that regulate protein translation, energy metabolism, lipid synthesis, autophagy and lysosome biogenesis.

mTORC2 specific subunits are Rictor (rapamycin-insensitive companion of mTOR), mSin1(mammalian stress-activated Map kinase-interacting protein1) and Protor (protein observed with Rictor 1/2). mSIN1 is a scaffolding protein for the downstream serum/glucocorticoid-regulated kinase 1 (SGK-1), Rictor is a scaffolding protein for the assembly of the whole complex and Protor1/2 facilitates the activation of SGK-1. mTORC2 is sensitive to growth factors which control survival, apoptosis, proliferation and cell shape.

\subsection{2 mTORC1 effectors}

Among the known targets of $\mathrm{mTORC} 1$ are translational regulators like 'eukaryotic translation initiation factor 4E-binding proteins' (4E-BP1/2/3) and the 'p70 ribosomal S6 kinase 1 and 2' (S6K1/2). The binding of 4E-BPs to 'eukaryotic translation initiation factor 4E' (elF4E) inhibits cap-dependent translation of mRNAs. The activation of mTORC1 phosphorylates the 4E-BPs and releases the inhibition of translation (Thoreen et al. 2012). The S6K1/2 phosphorylates a wide range of targets 
to positively regulate translation, including the $\mathrm{S} 6$ subunit of ribosomes, eukaryotic elongation factor-2 kinase (eEF2K), elF4B, S6K1 Aly/REF (SKAR)-like substrate and cap-binding protein 80 (CBP80) (Zoncu et al. 2011).

Simultaneously activated S6K1 promotes the transcription of 5 S ribosomal RNA and transfer RNAs and thereby contributes to ribosome biogenesis and protein translation (ladevaia et al., 2014). mTORC1 contributes to protein translation in an indirect fashion by inducing the expression of proteasome genes. This leads to increased proteasomal degradation which increases amino acid concentrations in the cell (Laplante and Sabatini 2012; Zhang et al. 2014).

Even though operating downstream of mTORC2, mTORC1 activity can alleviate mTORC2 by negative feedback regulation of S6K1 onto insulin receptor substrate (IRS). S6K1 phosphorylates IRS at S270 and S1101, priming it for sequestration, thus mediating insulin resistance (Ueno et al. 2005; Zhang et al. 2008).

In contrast, a positive feedback mechanism was described by Julien and colleagues (2010), in which S6K1 phosphorylates Rictor at T1135 which increases Akt phosphorylation by mTORC2.

mTORC1 is an effective activator of lipid metabolism. S6K1 mediated phosphorylation of transcription factor 'sterol regulatory element-binding protein-1/2' (SREBP1/2) induces the expression of several genes that are involved in fatty acid and cholesterol synthesis. Furthermore, adipogenesis-inducing 'peroxisome proliferator-activated receptor- $\gamma$ ' (PPAR- $\gamma$ ) is activated by SREBP1 (Peterson et al. 2011).

Catabolic processes like mitophagy or macroautophagy that are activated by starvation are negatively regulated by $\mathrm{mTORC} 1$ activity. mTORC1 directly inhibits the action of autophagy-inducing ULK1/Atg13/FIP200 complex (Dunlop and Tee 2014).

In cancer cells, mTORC1 activity increases transcription and subsequent translation of hypoxia inducible factor $1 \alpha$ (HIF1 $\alpha)$ that promotes glucose metabolism, cell proliferation and angiogenesis (Hudson et al. 2002). 


\subsection{3 mTORC2 effectors}

The central protein kinase Akt is downstream of mTORC2 activation. mTORC2 phosphorylates Akt at S473 which renders Akt fully active (Sarbassov 2005). This phosphorylation event is required for some of the Akt substrates such as forkhead box $\mathrm{O}$ (FoxO) transcription factors. Akt phosphorylates FoxO proteins at residues critical for nuclear export which removes FoxO from the nucleus and annihilates its signalling (Guertin et al. 2006). In parallel, mTORC2-induced eradication of nuclear FoxO proteins can be mediated by serum- and glucocorticoid-induced protein kinase 1 (Garcia-Martinez and Alessi, 2008; Pierce et al., 2011). Another target of Akt is the TCS1/2 complex. Akt phosphorylation of TSC2 inhibits its GTPase activity for 'Ras homolog enriched in the brain' (Rheb). Rheb itself activates mTORC1 by an unknown mechanism making mTORC2 ultimately an upstream activator of mTORC1 (Inoki et al. 2003).

A major function of mTORC2 is the regulation of actin polymerization by activation of PKC and Rac1 (Guertin et al. 2006; Thomanetz et al. 2013). One of these mechanisms involves recruitment of the GEF P-Rex1 into mTORC2 and subsequent activation of Rac1 (Hernandez-Negrete et al. 2007). Rac1 is a member of the Rac subfamily of the Rho family of GTPases. Rac1 is a pleiotropic regulator of many cellular processes including actin polymerization, lamellipodia formation and cell migration.

\subsubsection{Activation of mTORC1}

Most of the activating cues for mTORC1 come from extracellular signals. They are transmitted through cytokine receptors, ion channels, $\mathrm{G}$ protein-coupled receptors and receptor tyrosine kinases, and converge onto the TSC1/TSC2 complex. This dimeric complex of TSC1 (hamartin) and TSC2 (tuberin) represents a GAP for the mTORC1-activating Rheb. So far there is no known GEF for Rheb that could recharge inactive Rheb-GDP with GTP (Long et al. 2005). The TSC1/2 complex is targeted by central kinases like Akt, IKB kinase $\beta$ (IKKB), extracellular signalregulated kinase (ERK) and ribosomal S6 kinase (RSK) which all result in phosphorylation and subsequent inhibition of TSC1/TSC2 activity and thereby to a disinhibition of mTORC1 formation (Inoki et al. 2003; Huang and Manning 2008). 
An important modulator of the PI3K/Akt/mTOR signalling axis is the lipid 'phosphatase and tensin homolog' (PTEN). It counteracts the action of PI3K as it hydrolyses phosphatidylinositol-(3,4,5)-triphosphate at the plasma membrane to interrupt the activation of Akt.

Another important activator of mTORC1 signalling is cyclic adenosine monophosphate (cAMP). cAMP levels regulate the formation of $\mathrm{mTORC1}$, probably by a protein kinase A (PKA) mediated inactivation of PRAS40 or via the release of Rheb from sequestration by phosphodiesterase 4D (PDE4D) (Blancquaert et al. 2010; Kim et al. 2010).

Activation of mTORC1 by amino acids is facilitated by the activity of four different regulatory protein complexes and the recruitment of $\mathrm{mTORC} 1$ to the lysosomal compartment by Rheb. Rag family GTPases RagA or RagB form obligate heterodimers with RagC or RagD. Each Rag dimer-subunits binds either GTP or GDP depending on the amino acid concentration. Amino acid stimulation promotes the formation of $R a g A / B^{G T P}-$ RagC/D ${ }^{G D P}$ dimers which activate mTORC1 while amino acid deprivation promotes the formation of RagA/B ${ }^{G D P}-$ RagC/D ${ }^{G T P}$ dimers that inhibit $\mathrm{mTORC} 1$ formation. Prerequisite for this regulation is the recruitment of $\mathrm{mTORC} 1$ to LAMP1/2-positive lysosomal compartments via farnesylated Rheb. There mTORC1, interacts with the Rag heterodimers and the inhibitory Ragulator complex. Ragulator exhibits GEF activity and activates RagA/B at the lysosome. Inversely, a regulatory protein complex called GATOR1, possesses GAP activity towards RagA/B and inactivates Rag heterodimers. A third regulatory protein complex called GATOR2 suppresses GATOR1. Inactivation of GATOR1 renders mTORC1 insensitive to amino acid deprivation while inactivation of GATOR2 can suppress mTORC1 signalling (Sancak et al. 2010; Zoncu et al. 2011; Bar-Peled et al. 2013). 


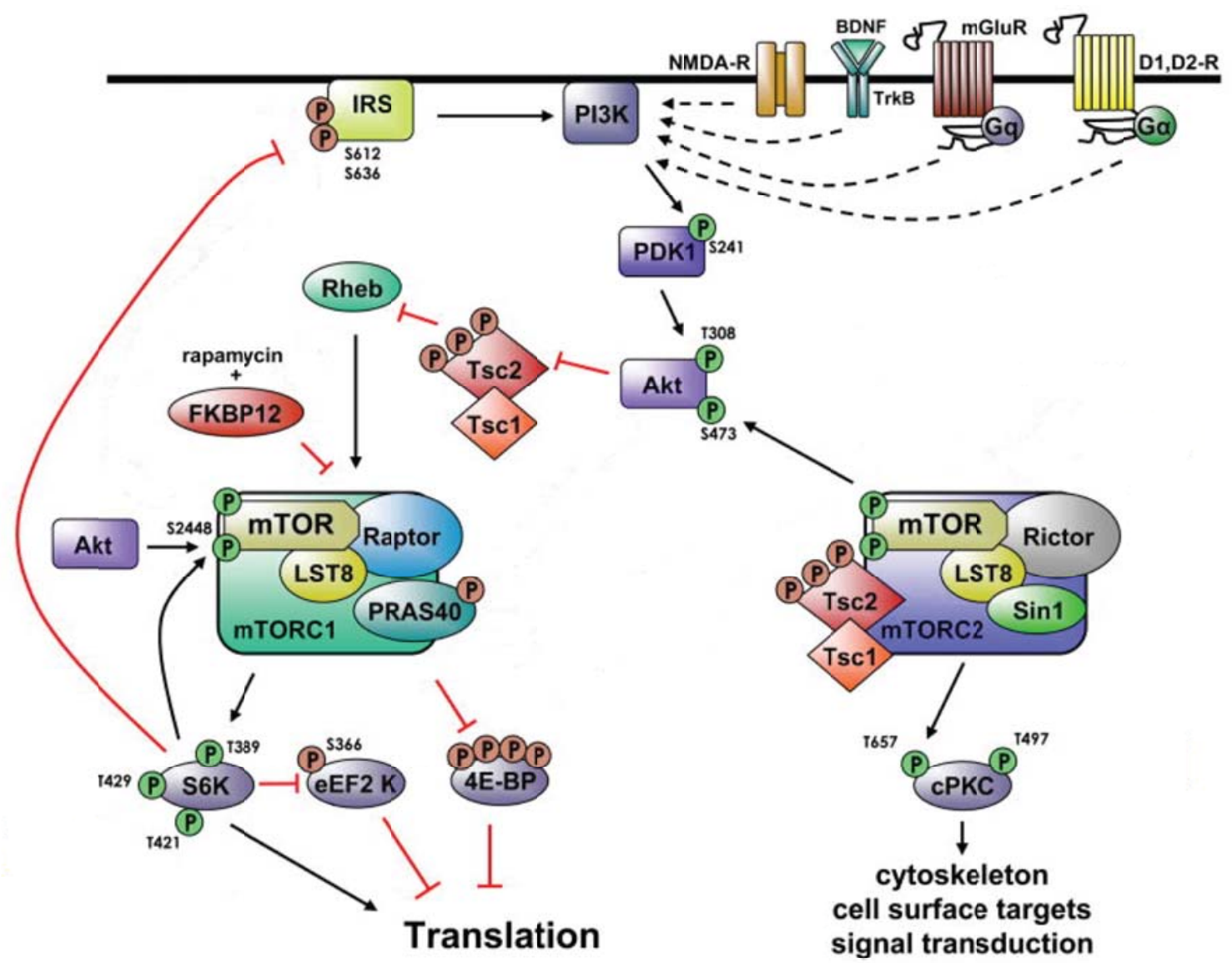

Figure 3: Activators and effectors of mTORC1 and mTORC2

mTOR forms two functionally and structurally distinct complexes, mTORC1 and mTORC2. Neuronal receptors and channels NMDA-R, TrkB, mGluR, D1, D2-R activate mTORC1 by the activation of PI3K, PDK1 and Akt. Akt becomes fully activated by mTORC2 and activates mTORC1 by inhibitory phosphorylation at TSC1/TSC2 complex which allows binding of Rheb to mTOR. The mTORC1 specific inhibitor rapamycin binds to FKBP12 and inactivates mTORC1. A positive feedback regulation by S6K1 phosphorylates mTOR at S2448. mTORC1 regulates cap-dependent mRNA translation via activation of S6K1 and inhibition of 4E-BP. mTORC2 regulates cytoskeleton rearrangements and cellular survival via activation of PKC. red $(P)$ denotes inhibitory phosphorylation, green $(P)$ denotes stimulatory phosphorylation. Phosphorylated residues are given as numbers. Modified from Hoeffer and Klann, 2010. 


\subsubsection{Inhibition of mTORC1}

Low energy levels are a major inhibitory factor for mTORC1 signalling. Cells limit energy-consuming processes like protein translation upon starvation. High concentrations of AMP activate '5'-AMP-activated protein kinase' (AMPK) which is a potent inhibitor of mTORC1 formation and functions as a major antagonist of Akt signalling. Activated AMPK phosphorylates TSC2 at S1345 and T1227 which enhances TSC1/2 inhibitory activity towards Rheb. In parallel AMPK phosphorylates Raptor at two inhibitory serine residues and renders it inactive (Gwinn et al., 2008). The action of AMPK therefore represents a metabolic checkpoint coordinating cell growth with energy status (Inoki et al. 2003).

Hypoxic conditions lead to high AMP/ATP levels but AMPK activation seems to be independent of that. Instead, stabilisation of the transcription factor 'hypoxia induced

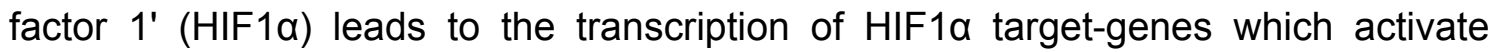
TSC1/TSC2 complex (Inoki et al. 2005). Moreover high reactive oxygen species levels, observed under hypoxic conditions are able to inactivate mTORC1 at the peroxisome and the mitochondrial level (Li et al. 2010; Benjamin and Hall 2013). And finally DNA damage can initiate p53-dependent transcription of TSC2 and PTEN but in parallel also directly activated TSC1/TSC2 complex via GADD34, which counteracts the effect of IKKB, RSK and Akt-dependent inactivation of the TSC2.

\subsubsection{Activation and inhibition of mTORC2}

Little is known about the upstream activators of mTORC2. In Dictyostelium dicoideum, G-protein coupled receptors (GPCRs) mediate chemotactic signals which specifically regulate mTORC2 independently of PI3K (Lee et al. 2005; Kamimura et al. 2008). Moreover, the association with the TSC1/2 complex and ribosomes appear to regulate mTORC2 positively (Zinzalla et al. 2011).

It has been described that mTORC2 is inhibited by mTORC1 overactivation due to a direct phosphorylation of Rictor by S6K1 (Julien et al. 2010). 


\subsection{7 mTOR complexes in brain physiology}

The expression and subcellular localization of neuronal mTOR and its complexes has not been studied in detail yet. However, there are data from non-neuronal cells that suggests that active mTORC1 is targeted to cellular organells by its interaction partners Rheb, Gator complexes, Ragulator and VATPase. Rheb, the major activator of $\mathrm{mTORC} 1$, is farnesylated and therefore likely to be targeted to intracellular membranes such as mitochondria, peroxisomes, lysosomes, late endosomes or the Golgi apparatus. Moreover, Rheb directly interacts with plasma membrane receptors like the NMDA receptor. In order to understand the mTORC1 and mTORC2 dependent signalling pathways better in context of neuronal cells, detailed knowledge about their subcellular localization and activity is necessary.

Aberrant mTORC1 signalling has been implicated in multiple physiological and pathological states throughout the nervous system. Neurotoxins that induce the disruption of calcium homeostasis have shown to inactivate mTORC1. In contrast, the overactivation of Akt, Rheb or a disruption of PTEN and consequently an overactivation of mTORC1 has been observed in numerous brain pathologies like tuberous sclerosis, epilepsy, schizophrenia or autism spectrum diseases. The Induction of autophagy by suppression of mTORC1 signalling has been shown to be beneficial in treatment of these pathological processes.

Neurons of the peripheral nerve system are able to regenerate after injury by axonal sprouting and healing due to increased mTORC1 signalling. In contrast, in several models of central nervous system neuron injury a downregulation of mTORC1 signalling has been observed, which results in the inability of regeneration and healing. Axon sprouting and healing can be induced in these models by activation of mTORC1.

\subsubsection{Dysregulation of mTOR in neurodevelopmental disease}

Neurodevelopmental disorders are impairments of the growth and development of the central nervous system. A more narrow definition considers disorders that affect brain functions like emotions, learning ability, self-control and memory that are manifested during growth of an individual. Autism spectrum disorders (ASDs) represent the biggest group of neurodevelopmental disorders. Affected individuals 
present common symptoms like impaired social interactions, abnormal repetitive behaviours and intellectual disability. Usually there is a genetic background with some forms of ASDs being linked to single genes. ASDs are heritable but they also occur spontaneously within the germline.

Some ASDs could be connected to mutations in negative regulators of mTORC1 signalling like TSC1, TSC2 or PTEN and mouse models of these diseases exist that partially reproduce the disease phenotype. These models were used to assess the potential of pharmacological modulation of $\mathrm{mTORC} 1$ signalling in respect to improvement of autistic behaviours, epilepsy, memory or learning.

Rapamycin has shown to reverse some of the behavioural and anatomical abnormalities in mouse models of neurodevelopemental disorders. Prolonged treatment with rapamycin in adult TSC2 +/- mice for instance improves the deficient cognitive and plasticity phenotypes (Chen et al. 1995; Cota et al. 2008).

The conditional inactivation of TSC1 in astrocytes, neurons or microglia leads to astrogliosis, macroencephaly, seizures and premature death (Crino et al. 2006; Cunningham et al. 2007; Crews et al. 2010; Aryal et al. 2014). Mice with conditional inactivation of TSC2 in radial glia develop astrogliosis, lamination defects, enlarged dysmorphic neurons and premature death. The chronic treatment with rapamycin has shown to be beneficial as it decreases the frequency of seizures and improves the lifespan of these mice.

ASD patients carrying mutations in their TSC1 or TSC2 gene show a loss of cerebellar Purkinje cells. Deletion of TSC1 in Purkinje cells effects social interaction and causes repetitive behaviour in mice, which recapitulated some of the behavioural deficits in humans. These mice also proof that behaviour is in part modulated by the cerebellum.

Conditional inactivation of PTEN in the brain leads to hypertrophic neuronal phenotypes, macroencephaly and seizures (Durán and Hall 2012; Dunlop and Tee 2014). Neuron-specific PTEN inactivation leads to abnormal social behaviour, impaired spatial learning, seizures and morphological changes that can in part be rescued by administration of rapamycin. Functionally PTEN inactivation has predominantly impact on long term potentiation (LTP) and long term depression 
(LTD). Also postnatal deletion of PTEN has shown to block synaptic plasticity but has no impact on the neuronal or synaptic structures per se.

Synaptic plasticity leads to either a long-term increase or a long-term decrease in synaptic transmission and are results of neuronal activity. It can be ectopically induced by electrical or pharmacological stimulation. LTD and LTP are believed to be cellular correlates of memory formation. LTP is classically divided into two stages, an early and a late stage. The early stage is characterized by rapid changes of posttranslational modifications like phosphorylation of the pre-existing synaptic components. The later stage shows changes in the synaptic content and requires protein translation, degradation and cytoskeletal rearrangements. In CA1 neurons late LTP is triggered by NMDAR activity and subsequent activation of mTORC1 and its effector kinase S6K. This activation seems to be spatially organized since it happens mostly in dendrites and to a less extend in spines but not in the cell body. The late stage induction of translation depends on the early stage activation of mTORC1 since it can be inhibited by administration of rapamycin.

The activation of mTORC1 by NMDAR activity is thought to be mediated by calcium influx through voltage dependent calcium channels and thus local increase of cAMP concentration. But also an indirect effect of autocrine BDNF signalling via the Trk- $\beta$ and the canonical PI3K-Akt-mTOR pathway is possible.

\subsubsection{Epilepsy}

Epilepsy is chronic neurologic disorder and one of the most frequent symptoms in neurodevelopmental disorders. Epileptic seizures, in absence of other associated pathologies increase mTORC1 signalling, a positive feedback mechanism that could contribute to progressive epileptogenesis. A classical morphological feature in patients with temporal lobe epilepsy is axonal sprouting of dentate granule cells. Rapamycin administration beginning directly after a seizure reduces axonal spouting, seizure frequency and neuronal cell death but has no effect on neurogenesis (Zeng et al. 2009; Buckmaster et al. 2009; Huang et al. 2010).

The WAG/Rij rat is a model of absence epilepsy. mTORC1 signalling is upregulated in the hippocampus of these rats and rapamycin administration before onset of seizures permanently reduces the development of seizures in this model, suggesting 
that mTORC1 overactivation might be a trigger of epileptogenesis (Russo et al. 2014).

The neuron specific as well as the astrocyte specific conditional TSC1 and TSC2 inactivation model that recapitulates symptoms of TSC patients, frequent spontaneous seizures can be reduced by rapamycin. In the animal model as well as in the patient reoccurring seizures are the most disabling neurological symptom. The cause for seizures is decreased activity of GABAergic neurons which results in network hyperexcitability. The connection between reduced GABA activity and mTORC1 signalling is not yet known.

\subsubsection{Depression}

Alteration in mTORC1 signalling have been implicated in clinical depression one of the most frequent psychiatric disorders. This implication represents a new angle in mTORC1 signalling since mTORC1 seems to be downregulated in depression.

Chronic stress as well as by long-term corticosterone treatment can inhibit the PI3KAkt-mTOR signalling pathway (Howell et al. 2011). Also cyclosporin A or tacrolismus chronically injected into the prefrontal cortex of rats induces depressive-like behaviours that can be reversed by NMDA-mediated activation of mTORC1 (Yu et al. 2013). Ketamine, a potent NMDA receptor antagonist is known to elicit rapid and long-lasting antidepressant effect. mTORC1 has been identified as a specific downstream effector of ketamine. Ketamine inhibits NMDA receptors preferentially on GABAergic interneurons, which leads to a disinhibition of glutamatergic neurons, increased depolarization and BDNF release. BDNF activates $\mathrm{mTORC} 1$ by binding to Trk- $\beta$ receptor and thereby starting the PI3K-Akt-mTOR signalling pathway which increases the translation of synaptic proteins like PSD95 and glutamate receptors.

\subsubsection{Rett syndrome}

Rett syndrome is a neurodevelopmental disorder that almost exclusively affects girls with a prevalence of 1 in 10000 births (Hagberg et al. 1983). It was first described by Andreas Rett, an Austrian paediatrician in 1966. Even though age of onset and manifestation of symptoms may vary, the characteristic feature of Rett syndrome is 
that a period of apparently normal development is followed by a developmental stagnation which pursues into a phase of regression. Early symptoms are an arrest of head growth which usually leads to microencephaly, reduction or loss of acquired skills such as purposeful use of the hands, vocalization and communication skills. Another characteristic of the disease are the intense and continuous stereotypical hand movements that appear after loss of purposeful hand movements. Moreover breathing irregularities, autism-like behavior, seizures, growth retardation and cardiac abnormalities are key features of the disease (Shahbazian and Zoghbi 2001).

Rett syndrome can be divided into 4 stages. The onset of the first stage is between 6 and 18th month of age and is characterized by a developmental stagnation that may not be noticed immediately. The second stage usually begins between 1 and 3 years and shows a rapid developmental degression. The infant loses purposeful hand movements and language. Stereotypical hand movements such as wringing, washing and mouthing may start during this phase. Other symptoms appearing in this state are breathing irregularities characterized by episodes of hyperventilation and apnoea, social withdrawal, loss of communication and decreased motoric abilities. The following third stage is characterized by a pseudo-stationary state. Prominent features are generalized apraxia and reoccurring seizures. This stage can last for years and may show improvement in the autistic behavioural features. The fourth stage, or motor deterioration-phase, can last for years or even decades. It is characterized by muscle weakness, rigidity, spasticity, dystonia and scoliosis and hence loss of mobility. Stereotypic hand movements usually become simpler in this stage and general eye contact and communication remain intact (Weaving et al. 2005).

\subsubsection{Genetic background of Rett syndrome}

Rett syndrome is caused by mutations in the methyl CpG binding protein 2 (MeCP2) encoding gene located at the X-chromosome. In $80-90 \%$ of all cases these are denovo mutations (Shahbazian and Zoghbi 2001). MeCP2 is a transcriptional regulator that binds to 5-methylcytosine, methylated cytosines within cytosin-phosphateguanine dinucleotides (Lewis et al. 1992). Ironically the majority of reported 
mutations in MECP2 are $\mathrm{C}$ to $\mathrm{T}$ mutations within one of the eight $\mathrm{CpG}$ dinucleotides (Lee et al., 2001).

Due to random X-inactivation girls with RTT represent a mosaic of healthy and diseased cells on tissue level. However skewed X-activation leading to non-random inactivation, favouring one of the $\mathrm{X}$-chromosomes may explain the high variability in RTT phenotype manifestation (Plenge et al. 2002; Weaving et al. 2005).

\subsubsection{MeCP2 protein function}

MeCP2 is a transcriptional regulator that binds to methylated cytosines in CpG dinucleotides. $\mathrm{CpG}$ dinucleotides are often found in the promoter region of genes and in heterochromatin. The methylation of $\mathrm{CpGs}$ inside promoter regions usually leads to silencing of that particular gene. In contrast, hypomethylation of CpGs has been associated with protein overexpression (Meehan et al. 1989).

The human MECP2 gene comprises if 4 exons and gives rise to 2 isoforms by alternative splicing. The two isoforms are very similar as they share all the functional domains but they vary at the start. The m-RNA of isoform MeCP2e1 comprises of exon 1, 3 and 4 while mRNA of isoform MeCP2e2 comprises of exon 2, 3 and 4 . Isoform 1 is considered to be the predominant isoform expressed in the brain (Dragich et al. 2007). The protein harbours three functional domains: a methyl-CpGbinding domain (MDB), a transcriptional repressor domain and a nuclear localization sequence (Lewis et al. 1992). MeCP2 can repress transcription by binding to methylated $\mathrm{CpG}$ dinucleotides and subsequent recruitment of co-repressor $\operatorname{Sin} 3 \mathrm{~A}$ and histone deacetylase (HDAC) and BRM ATPase (Vecsler et al. 2010). This complex promotes the deacetylation of histones and leads to silencing of the concerned promoter. Phosphorylated MeCP2 that binds to CDK5 cannot bind to methylated $\mathrm{CpG}$ dinucleotides and therefore fails to exert silencing effect on that particular gene.

Furthermore MeCP2 associates with the transcriptional activator CREB1 at promoters of activated but not repressed targets in the hypothalamus. The expression of the vast majority of genes in the hypothalamus seems to be regulated positively by $\mathrm{MeCP} 2$, which lead to a revision of MeCP2's role as transcriptional repressor (Chahrour et al. 2008). 
The brain is the most affected by MeCP2 dysfunction. It is also the place where its expression is highest. Moreover the fact that the MeCP2 expression closely coincides the postnatal maturation of the brain is the reason for people to believe that MeCP2 is involved in neuronal and synaptic maturation and maintenance. Several neuronal genes have been observed to be dysregulated upon MeCP2 inactivation in mice (Pelka et al. 2006; Smrt et al. 2007). One of the most prominent ones is Bdnf, encoding brain derived neurotrophic factor (BDNF). BDNF is a neurotrophin and potent modulator of neuronal development, synaptic transmission and plasticity. A loss of BDNF function leads to severe neurodevelopmental, neurodegenerative and neuropsychiatric diseases (Zuccato and Cattaneo 2009; Autry and Monteggia 2012). In rodents BDNF expression closely coincides MeCP2 expression. Conditional deletion of BDNF in forebrain excitatory neurons results in phenotypes similar to those in MeCP2 null mice, such as hind limp clasping, decreased brain weight, smaller olfactory and hippocampal neurons (Guy et al. 2001; Chang et al. 2006). The finding of BDNF expression being controlled by MeCP2 would also explain the general brain atrophy, reduction in dendritic arborisation as well as reduced number of synapses observed in the MeCP2 null mice.

There are two models that aim to explain how MeCP2 controls transcription of Bdnf One of them being the repression model, based on the finding that MeCP2 binds to Bdnf promotor IV in cultured hippocampal neurons and prevents transcription. Upon depolarisation, MeCP2 is phosphorylated and subsequently released from the promoter site to allow transcription of Bdnf. Neuronal activity causes fast phosphorylation of cAMP response element binding protein (CREB1) which is a requirement of BDNF expression. Other mechanisms that underly the enhancement of Bdnf transcription upon MeCP2 unbinding are DNA demethylation and unbinding of co-repressors. The repression model fails to explain the fact that BDNF protein expression is lower in MeCP2 knockout mice. In the hypothalamus BDNF levels correlate with the expression of MeCP2, being lower in the MeCP2-KO and higher in the MeCP2 overexpressing mice when compared to wildtype (WT) controls (Chahrour et al., 2008).

Long-term potentiation is a form of synaptic plasticity that relies on long-term changes in synapse anatomy and relies on de-novo synthesis of synaptic proteins. It is considered to underlie memory formation. Impairment in LTP induction and 
maintenance has been correlated with general learning and memory deficits. Electrophysiological studies in hippocampal slices of MeCP2-KO mice revealed impairments in LTP compared to WT mice (Asaka et al. 2006).

Several studies have demonstrated that BDNF participates in synaptic plasticity and is critical for dendritic spine formation and maturation during development (Poo 2001; Tyler 2002; Tanaka et al. 2008; Cohen et al. 2011). Exogenously applied BDNF increases spine density in cultured hippocampal neurons and CA1 pyramidal neurons in slice cultures (Tyler and Pozzo-Miller 2001; Ji et al. 2005). BDNF application also shifts the proportion of morphological types of spines. Overexpression of BDNF can rescue the dendritic atrophy caused by transient knockdown of MeCP2 in cultured hippocampal neurons. At dendrites BDNF binds to tropomyosin related kinase B (TrkB) receptor and activates ERK, PI3K and PLC-Y. Dendritic spine anomalies have been identified in multiple brain regions in RTT and MeCP2 inactivation models. Since BDNF promotes the formation, maintenance and activity-dependent remodelling of dendritic spines, its reduced expression upon MeCP2 inactivation is viewed as the major factor of the neurological dysfunction is RTT.

\subsection{FRET}

First described by Theodore Förster over 60 years ago, Förster resonance energy transfer (FRET) is a dipole-dipole interaction that occurs between two fluorophores that possess a spectral overlap between the donor's emission and acceptor's absorbance (Figure 4a). It is important to note that the transfer of energy is nonradiative, i.e. it does not involve emission and re-absorption of photons (Förster 1948). The rate of energy transfer that occurs via FRET is described in the equation (Lakowicz 2006):

$$
K_{F R E T}=\left(\frac{1}{\tau_{D}}\right)\left(\frac{R_{0}}{R}\right)^{6}
$$

where $\tau_{D}$ is the excited state lifetime of the donor and $R$ is the distance separating the fluorophores. As the FRET critical distance ( $R_{0}$ - Förster distance) between two fluorophores correlates inversely to the $6^{\text {th }}$ power of separation distance, the FRET efficiency $(\mathrm{E})$ dramatically decreases with increasing distance in the vicinity of $R_{0}$ 
(Figure 4b) (Berney and Danuser 2003; Lakowicz 2006). The $R_{0}$ distance is defined by the spectral properties of the FRET pair, and represents the distance at which $E$ is 50\% (Lakowicz 2006):

$$
R_{0}^{6}=8.79 * 10^{-5}\left[\kappa^{2} J(\lambda) n^{-4} Q_{D}\right]
$$

where $\kappa^{2}$ is the orientation factor between the two fluorophores, $Q_{D}$ is the donor quantum yield and $J(\lambda)$ represents the overlap of donor emission and acceptor absorbance spectra. The overlap integral, $J(\lambda)$ is defined as (Lakowicz 2006):

$$
J(\lambda)=\int F_{D}(\lambda) \varepsilon_{A}(\lambda) \lambda^{4} d \lambda
$$

where $F_{D}(\lambda)$ is the normalised fluorescence emission and $\varepsilon_{A}(\lambda)$ is the molar extinction coefficient of the acceptor absorbance spectrum (Lakowicz 2006).

Distances between 1 - $10 \mathrm{~nm}$ can be measured using FRET, making its application in cellular imaging ideal for the visualisation of interactions on the $\mathrm{nm}$ length scale (Wu and Brand 1994; Clegg 1995; Patterson et al. 2000; Akrap et al. 2010). The continued development of fluorophores has enabled FRET applications in life sciences, where elucidation of signalling pathways and/or partners is often dependent on the ability to resolve transient interactions of multiple biomolecules.

In order to 'pair' fluorophores for use in measuring FRET, they must fulfil a number of criteria. Firstly, for optimal FRET, the spectral overlap between donor fluorescence and acceptor absorbance must be as large/extensive as possible. Additionally, the donor quantum yield and acceptor extinction coefficient must be as high as possible. Intensity-based FRET furthermore requires a bright acceptor so that the FRETinduced 'sensitised' emission can be easily detected. Lastly, the angular orientation of dipole transition moments of the donor and acceptor fluorophores is an important factor in the efficiency of FRET. This is described by the orientation factor $\kappa^{2}$ in the description of $R_{0}$, and it's values can range between 0 and 4 . A value of 0 corresponds to a perpendicular orientation of donor and acceptor dipole moments, which is not capable of FRET, and 4 corresponds to a collinear orientation which results in maximal FRET efficiency. In a completely averaging regime, where fluorophores are assumed to possess complete rotational freedom, the statistical average over all sampled orientations amounts to a value for $\kappa^{2}$ of $2 / 3$. This value is typically used for the calculation of $R_{0}$ values in lists of FRET pairs. However, if the FRET pairs are connected to interacting proteins, steric restrictions apply that 
invalidate the assumption of a $k^{2}$ of $2 / 3$. Thus, the value for $R_{0}$ might significantly deviate from the expected values that are based on the averaging regime. This is also the reason why great care should be taken in the estimation of separation distances from FRET efficiencies.

In measuring FRET, these requirements are not always able to be optimised. Maximising spectral overlap introduces bleed-through of donor emission to the acceptor channel and the excitation of the acceptor at donor wavelengths, problems that require extensive correction in sensitised emission FRET (Berney and Danuser 2003). As a large section of the available spectral window is occupied when the emission of two fluorophores is monitored, the use of multiple FRET pairs often difficult (He et al. 2005; Ai et al. 2008) (Figure 4).

\subsection{FLIM}

FRET between donor and acceptor fluorophores quench the donor fluorescence in proportion to the efficiency of FRET as the fluorescence lifetime is proportional to the fluorophores' 'specific brightness', i.e. its quantum yield. This change in lifetime can be observed by fluorescence lifetime imaging microscopy (FLIM) (Bastiaens and Squire 1999; Lakowicz 2006).

As the fluorescence lifetime is an intrinsic property of a particular fluorophore and independent of fluorophore concentration, FLIM is advantageously compared to intensity-based FRET measurements. In contrast to intensity-based FRET measurements, donor lifetime measurements suffice to detect environmental changes, and the measurement puts no demands on the acceptor quantum yield characteristics (Bastiaens and Squire 1999; Chen et al. 2003). It is even possible, and in some cases beneficial, to utilise non-fluorescent acceptors to measure FRETFLIM (Ganesan et al. 2006). The use of 'dark' acceptors in FLIM measurements allows wider detection range for donor emission. More advanced FRET-FLIM measurements that involve the switching of the absorption properties of the acceptor, such as pcFRET, can be used (Subach et al. 2010; Petchprayoon and Marriott 2010; Don Paul et al. 2013).

FLIM is generally subdivided into time domain (TD) or frequency domain (FD) methods (Chen et al. 2003; van Munster and Gadella 2005). Both approaches follow 
the effects succeeding an excited fluorophore, but the acquisition and analysis of the data differ. In TD FLIM, fluorophores are excited by a train of very short discrete light pulses. The decay kinetics of the excited state are sampled by measuring the arrival time of the first photon with respect to the excitation pulse. FD FLIM, on the other hand, typically uses a periodically modulated light source, rather than light pulses, to determine the modulation in the emission signal that is generated by the duration of the excited state. This distortion causes changes in the time-integrated signal on a regular charge-coupled device (CCD) camera, from which the fluorescence lifetime can then be estimated. Both modes can be used in wide-field and scanning microscopes. Most common set-ups for FD FLIM utilise wide-field microscopy whilst TD FLIM is mostly based on laser scanning microscopy. The difference in methodology can be reflected in their biological applications with FD FLIM being faster and therefore applied to living cells, whilst TD FLIM requires longer acquisition times and therefore preferentially used in fixed samples. 

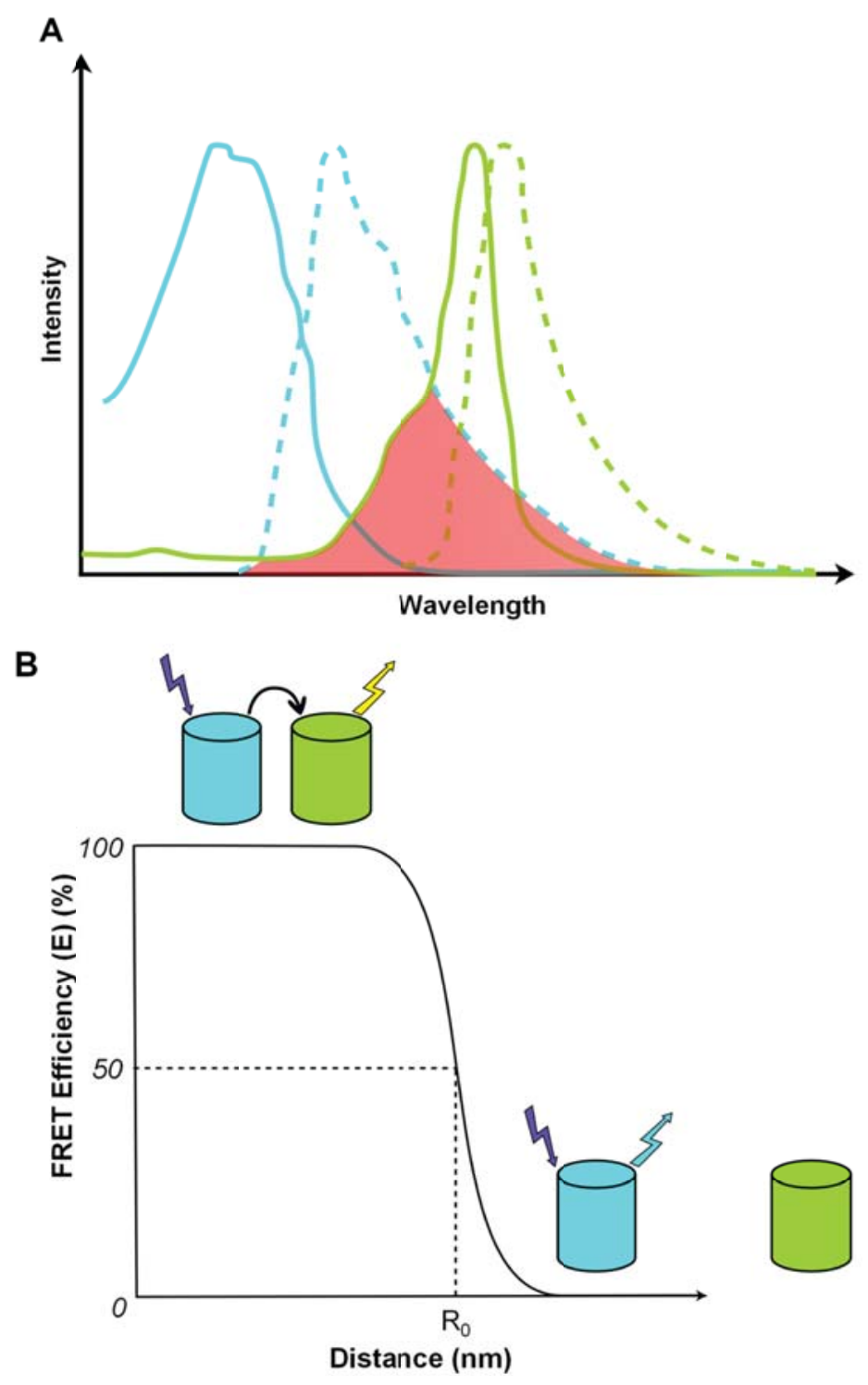

\section{Figure 4: Principle of FRET}

A: The CFP (cyan) and YFP (yellow) FRET-pair showing the excitation (solid line) and emission spectra (dashed line). Highlighted (red) is the spectral overlap between CFP emission and YFP absorbance/excitation, a requirement for FRET. B: Highlighting the distant dependent nature of FRET, the graph explains the exact nature of FRET and shows why it can be utilised as a 'molecular ruler'. The Förster distance $\left(R_{0}\right)$ value is calculated at 50 \% FRET efficience. Ebrecht and Don Paul, 2014. 


\subsubsection{Time domain FLIM techniques}

In TD FLIM, very short pulses (fs - ps) of light are used to sample the fluorescence decay of a fluorophore. Following data acquisition, the shape of the decay function is fitted to an exponential decay model to determine its fluorescence lifetime. The most common implementation is time-correlated single photon counting (TCSPC) where the arrival time of many single emitted photons are recorded with respect to the excitation laser pulse for the different scanned positions in the image.

Current detectors exhibit 'dead times', i.e. a delay time in which the detector cannot record a new photon, that are long with respect to the lifetime, typically in the $\mu \mathrm{s}$ range. As most fluorophores have fluorescence lifetimes of a few ns, a large number of photons are missed by the detector while it resets. Recording a time-resolved decay curve from a single excitation burst of a fluorophore would require extremely fast detectors with a time resolution in the tens of picoseconds. As no such detector currently exists, TCSPC FLIM uses a periodic excitation scheme extended over multiple excitation events. In this way, a decay curve is reconstructed from single photon events collected over many cycles (Figure 5). In order to unambiguously assign the emission photon to the excitation event, the emission probability per event is kept low. As not every excitation pulse generates a photon, the excitation pulse immediately following a detected photon incident is used as a time reference in a 'reverse start-stop' procedure.

One major disadvantage of TCSPC FLIM is the long acquisition time. It can take up to 10 minutes or longer to gather enough photons for a reliable lifetime fitting procedure. In most cases the count rates of TCSPC FLIM systems are not the limiting factor. Count rates of TCSPC FLIM systems can range up to ten megacounts per second and is limited mainly by the photostability of the dye and the scanning speed of the microscope but not by the counting ability of the detection system (Katsoulidou et al. 2007; Becker et al. 2009). The optimisation for brightness of fluorophores like mCerulean3 (Markwardt et al. 2011) or mTurquoise2 (Goedhart et al. 2012) is one way to reduce the acquisition times. Additionally, the use of less photon-demanding fitting routines, e.g. Bayesian fitting (Rowley et al. 2011), can be used to reduce the acquisition times. 

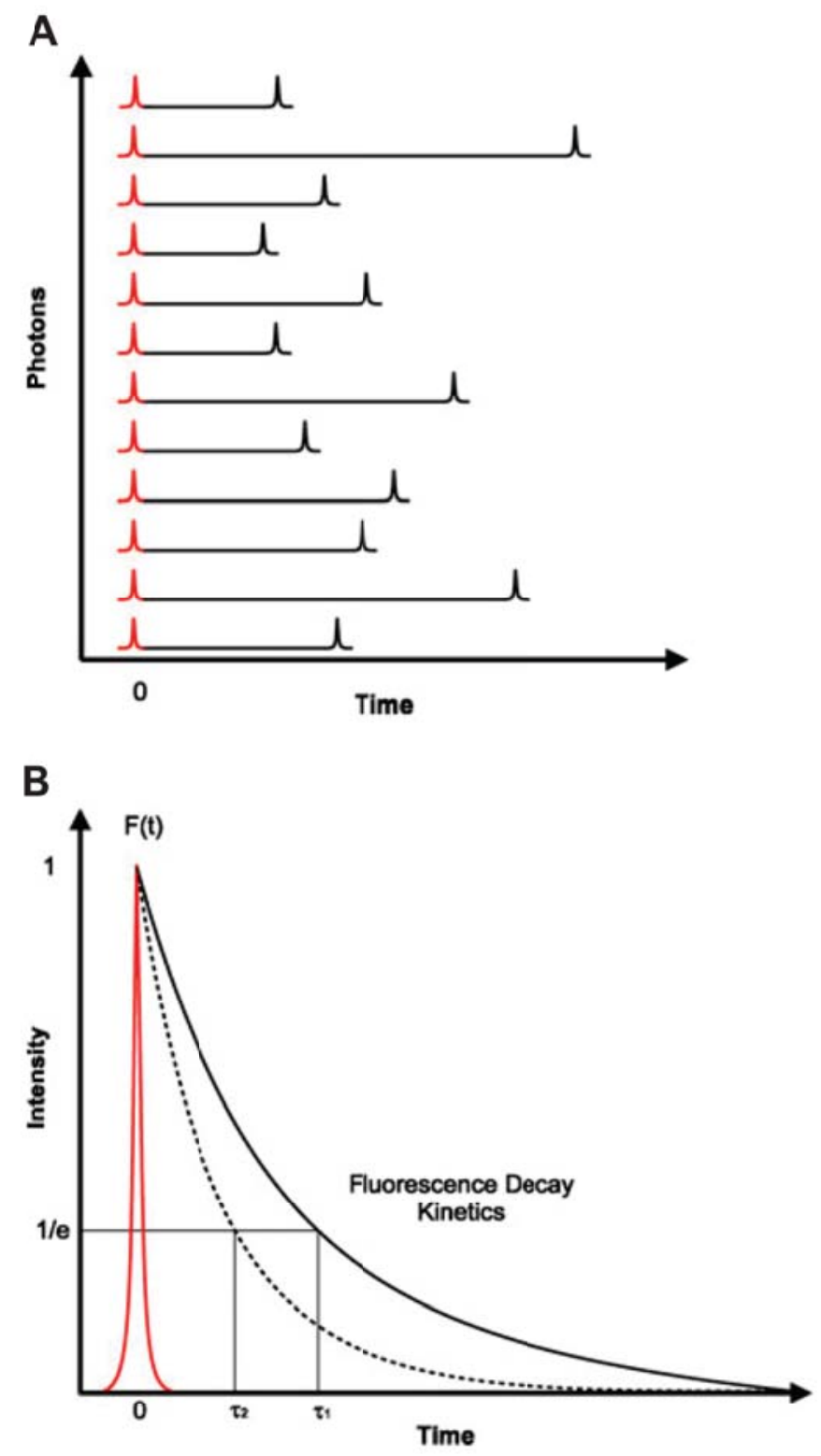

Figure 5: Principle of time correlated single photon counting FLIM

A: An initial short excitation pulse (red peak) excites fluorophores in a sample into an excited state. The time between the excitation pulse and detection of the emitted photon is recorded and this process repeated as many times as required to build an accurate decay profile. B: Following the recording of numerous arrival times of photons, a fluorescence decay kinetic profile can be calculated. A decrease $\mathrm{T}$ of a donor (solid line) indicates an interaction with another chromophore or quencher or is due to changed environmental factors (dashed line). The corresponding time at an intensity of $1 / \mathrm{e}$ denotes the fluorophore lifetime (dotted lines). Modified from Ebrecht and Don Paul, 2014. 
While TCSPC FLIM aims to reconstruct the fluorescent decay profile by timing single photon events, an alternative method of sampling decay kinetics after a brief light pulse is to record photons in consecutive time bins. At the core of a time-gated FLIM system is the image intensifier. On arrival of photons at the photocathode, photoelectrons are produced by the photoelectric effect, which are then multiplied thousand-fold in the multichannel plate before generating photons on the anode phosphor screen that are imaged by a CCD camera. The image intensifier can be gated in time with high temporal resolution by application of a gating pulse (DymokeBradshaw 1993). As the intensifier gating is synchronised with the pulsed excitation signal, the camera is opened in the same relative period in successive excitation cycles, gaining time-integrated signals of the decay at arbitrary total exposure times. The decay is sampled at two or more positions in time. In case of two time gates of equal width and a mono-exponential fluorescence decay, the fluorescence lifetime can be easily determined from the ratio of the recorded intensities by the rapid lifetime determination (RLD) formula (Ballew and Demas 1991):

$$
\tau=\frac{\Delta \tau}{\ln \left(I_{1} / I_{0}\right)}
$$

where $I_{0}$ and $I_{1}$ represent the images recorded in the first and second time bin and $\Delta T$ is the gating time. For multi-exponential decay kinetics, the time-gating implementation with two time gates yields an average lifetime, which is sufficient to provide lifetime contrast of biological probes. More than two time gates have been shown to provide quantitative results for biological samples containing multiexponential decays (Scully et al. 1997; Esposito and Wouters 2004).

Time-gated image intensifiers can be used with scanning microscopes (Wang et al. 1991; Cole et al. 2001) but more interesting is the combination with wide-field set-ups because the CCD chip based detection system allows the simultaneous acquisition of all spatial information at once, translating in an increase in acquisition speed compared to sampling the image by scanning (Wang et al. 1992; Scully et al. 1996; Dowling et al. 1997). A faster way to record time-gated FLIM on a scanned system is via multi-beam scanning as described by (Straub and Hell 1998).

The image analysis of the RLD method affords significantly shorter calculation time and is surprisingly robust and effective at providing lifetime-based contrasts in biological probes in real time (Ballew and Demas 1989; Esposito et al. 2007). The 
acquisition times are generally shorter than techniques based on spatial scanning. As the number of time gates is inversely proportional to photon counting efficiency, the more time gates used to sample the decay function, the fewer photons will be recorded.

The problem of photon efficiency can be eased by recording in only a few time gates in combination with a single-shot detection configuration. Single-shot detection splits an image into two or more images using a beam splitter and each image is designated to a different time bin. In one implementation, one of the two images was projected immediately onto the gated image intensifier while the other image was delayed by taking a detour of several meters before being projected onto the same detector (Agronskaia et al. 2003). For simultaneous, parallel detection, the system is equipped with a four-channel optical splitter working in conjunction with a segmented gated image intensifier. Unlike a conventional image intensifier, the photocathode is subdivided into four quadrants by resistive sectioning, providing four channels, which can be gated at different delay times. The image splitter can therefore relay four subimages of the sample onto four quadrants of the detector (Elson et al. 2004).

Despite the obvious speed advantage of gated wide-field FLIM setups, it should be noted that the lack of confocal out-of-focus light rejection can cause the mixing of fluorescent signals with different lifetime characteristics, thereby reducing lifetime contrast.

\subsubsection{TD FLIM image analysis}

Despite the relative simplicity and speed of the RLD method (Ballew and Demas 1989), it only estimates the average lifetime of all lifetime components weighted by their individual contribution to the mixture. Most FLIM applications in life sciences must deal with multi-exponential decays, derived from mixtures of fluorophores, spectrally similar but possessing different lifetimes. The average fluorescence lifetime can be useful in the case of FRET as it provides contrast between the quenched and unquenched donor. However, due to the fact that the decay curve is strongly under sampled, it is not possible to extract single lifetimes from multi-exponential decay curves. Consequently, determining fractions of donor molecules, which do or do not engage in FRET is not easily possible. 
The only technique providing direct access to parameters of multi-exponential decays is TCSPC FLIM. The data from TCSPC measurements usually consists of a large number of photons recorded in a many time channels for each pixel of an image. These photon numbers and corresponding time channels resemble fluorescence decay curves in each pixel of the image. Importantly, at this stage, the data is still a convolution of fluorophore data and the instrument response function (IRF). The IRF is the response of the detection system to only the excitation pulse and can be measured or calculated from the decay function by Fourier analysis. The deconvolved decay curve can then be fitted to a mathematical model of choice until the best fit is achieved (O'Connor 1984).

\subsubsection{Frequency Domain FLIM techniques}

FD FLIM employs an excitation light source which is periodically modulated in intensity rather than a train of very short excitation light pulses. The emitted fluorescence is shifted in phase and the amplitude is demodulated relative to the excitation light. The distortion in the temporal emission profile resulting from the time that a fluorophore spends in an excited state before emitting a photon is used to estimate the fluorescence lifetime (Figure 5). The modulation of the excitation can be a sinusoidal or block wave. Sine wave excitation will result in an emission sine wave of the same frequency, but shifted in phase $(\Phi)$ and with reduced amplitude. A block wave signal will lose higher-order frequencies as the emission will 'smear out' the sharp edges of the block wave. Provided that a lifetime is long enough, the block wave will result in a sine wave at the fundamental modulation frequency. These effects are described in (Lakowicz 2006):

$$
\begin{gathered}
\tau_{\varphi}=\frac{\tan (\varphi)}{\omega} \\
\tau_{M}=\sqrt{\frac{1-M}{(M \omega)}}
\end{gathered}
$$

where $T_{\Phi}$ is the phase lifetime, $\Phi$ the phase shift at every modulation frequency $\omega, T_{M}$ is the modulation lifetime and $\mathrm{M}$ is the modulation.

The emission function is therefore 'dampened' according to the fluorescence lifetime 
of the fluorophore. An analogue from elementary school physics helps us to intuitively understand the connection between lifetime, phase delay and the reduction in amplitude. Imagine a movable arm (modulated excitation) connected to a spring and a weight. Oscillation of the arm moves the weight and compared to the movement of the arm, movement of the weight is delayed in time and its amplitude is reduced. The parameter that defines delay and demodulation is the spring constant. In our model, it is equivalent to the fluorescence lifetime. A small spring constant/lifetime will result in a small phase delay while a large spring constant/lifetime will cause a large phase delay. The amplitude will be larger for small lifetimes and smaller for large lifetimes.

As biological fluorophores usually possess fluorescence lifetimes in the nanosecond range, the excitation intensity is modulated at tens of Megahertz. If the modulation frequency is too low, the fluorescence will decay before the excitation cycle is completed. If the excitation frequency is too high, excited fluorophores will still be emitting whilst the excitation cycle starts again leading to saturation and an averaging-out of the modulation. Different lifetime components in a sample therefore require multiple frequencies (Colyer et al. 2008). The lifetime of a fluorophore can be determined directly via its phase delay or modulation ratio at different modulation frequencies. For single exponential decays, if the decay kinetics are best fit to a single exponential decay function, the lifetime phase shift and modulation change will be the same at all frequencies. If the decay is multi-exponential, then the lifetime phase shift will be smaller than the lifetime modulation change and their values will depend on the modulation frequency. Therefore, FD FLIM provides immediate information on lifetime heterogeneity.

The high acquisition speeds of frequency domain FLIM make it an ideal technique for fluorescence lifetime measurements of living cells. One of the reasons why, time domain equipment is more widely implemented in laboratories and imaging facilities, is the relative complexity of frequency domain equipment. Particularly, the image intensifier is an expensive and vulnerable component that, additionally, degrades the image quality. 


\section{Aims of the thesis}

The intention of this thesis was to investigate the role of mTOR signalling in synapse formation and plasticity and its failure in Rett syndrome. mTOR signalling is compromised in mouse models of Rett syndrome. Therefore mTOR signalling was supposed to be analysed on single cell level in order to clarify the contribution of neuronal subpopulations in the expression of Rett syndrome.

Firstly, optical sensors were developed, optimised, and evaluated to address the activity state of endogenous mTORC1/2. These sensors were designed with the foresight to be used in combination with the detection neuronal identity markers, allowing cell-type specific analysis of mTORC1 and 2 activities.

Secondly, the role of mTOR in inhibitory synapse plasticity was to be elucidated. To this end, the interaction of mTOR with gephyrin, the major organiser of the inhibitory postsynapse, was studied and the aspects of this interaction were analysed.

And lastly, the clustering of gephyrin and its regulation was quantitatively analysed by creating FRET sensors that report on the interactions of the G-domain and E-domain of gephyrin. These sensors were combined with the expression of the major regulators of gephyrin clustering to quantify their influence on the clustering process. The role of $\mathrm{mTOR}$ signalling in gephyrin clustering was addressed by pharmacologic inhibition of mTORC1 and mTORC2. 


\section{Materials and Methods}

\subsection{Mice}

Animal care was conducted in the central animal facility of the University of Göttingen, according to institutional guidelines with a $12 \mathrm{~h}$ light/ $12 \mathrm{~h}$ dark cycle and ad libitum access to food and water. Genotypes of MeCP2 (B6129P2(C)-Mecp2 ${ }^{\mathrm{tm}-1-}$ 1 Bird, hereafter referred to as $\mathrm{MeCP}^{-/ Y}$ ) were determined prior to start of the experiment via polymerase chain reaction (PCR) from isolated tail tissue. Only male mice were used for experiments as the MeCP2 gene is located to the $X$ chromosome.

MeCP2 ${ }^{\text {tml.IBird }} \mathrm{KO}$ mice of 0-1 day postnatally were obtained and sacrificed by decapitation. WT littermate controls were used for all experiments. Hippocampus was used to prepare a dissociated cell culture.

\subsubsection{The MeCP2 ${ }^{\text {tml.IBird }} \mathrm{KO}$ mouse as a model of Rett syndrome}

Mice, lacking the MeCP2 gene (B6129P2(C)-Mecp2 ${ }^{\mathrm{tm}-1-1 \text { Bird }}$ ), were used as a model organism for Rett syndrome. They were bred with C57BL/6J WT males to generate heterozygous females, hemizygous males and WT mice of either gender.

\subsubsection{DNA isolation and genotyping of MeCP2 ${ }^{\text {tml.IBird }} \mathrm{KO}$ mouse}

\section{DNA isolation}

Tail biopsies of P0-P1 mice were digested in $350 \mu$ tail lysis buffer $(50 \mathrm{mM}$ Tris/ $\mathrm{HCl}$ pH 8.0, $100 \mathrm{mM} \mathrm{NaCl}, 10 \%$ SDS, $1 \mathrm{mM}$ EDTA pH 8.0, $200 \mu \mathrm{g}$ Proteinase K) for minimum 3 hours at $65^{\circ} \mathrm{C}$, shaking. Debris was pelleted for $10 \mathrm{~min}$ at 20,000 g. 300 $\mu \mathrm{l}$ supernatant was precipitated by adding $350 \mu \mathrm{l}$ isopropanol and pelleted at 20,000 $\mathrm{g}$ for $10 \mathrm{~min}$. The pellet was washed with $70 \%$ ethanol and dried for $30 \mathrm{~min}$ at $37^{\circ} \mathrm{C}$. The DNA pellet was solved in $150 \mu 10 \mathrm{mM}$ Tris/ $\mathrm{HCl}(\mathrm{pH} 7.5)$.

\section{Genotyping PCR according to Miralvès et al., 2007}

The PCR reactions were performed in a final volume of $20 \mu \mathrm{l}$ containing $20 \mathrm{ng}$ tail DNA. The PCR cocktail was made up of $1 \times$ NEB PCR Reaction Buffer (New England Biolabs Inc., USA), 2,5 mM MgCl2, $200 \mu \mathrm{M}$ dNTPs, $1 \mu \mathrm{M}$ primers (Sigma-Aldrich, 
USA) and 1 U Taq DNA polymerase (New England Biolabs Inc., USA). Primers used are shown below (Table 1) (Miralvès et al. 2007).

PCR conditions were as follows: denaturation at $95^{\circ} \mathrm{C}$ for 30 seconds, annealing at $64^{\circ} \mathrm{C}$ for 30 seconds and extension at $72^{\circ} \mathrm{C}$ for 40 seconds, followed by a final extension at $72^{\circ} \mathrm{C}$ for 7 minutes. The reactions were held at $4^{\circ} \mathrm{C}$ until analysis.

PCR products were all analysed by $1.5 \%$ agarose gel electrophoresis in $1 \times$ TAE buffer, stained with ethidium bromide (Roth, Germany) and photographed with a Peqlab transilluminator (Peqlab, Göttingen, Germany). The molecular weight marker used was 1000 bp DNA ladder (New England Biolabs Inc., USA).

Table 1: Genotyping primers

\begin{tabular}{|l|l|}
\hline Primer name & Primer sequence $\left(5^{\prime}-3^{\prime}\right)$ \\
\hline P3 WT FW & GACCCCTTGGGACTGAAGTT \\
\hline P3 KO FW & CCATGCGATAAGCTTGATGA \\
\hline P3 RV & CCACCCTCCAGTTTGGTTTA \\
\hline
\end{tabular}

\subsubsection{Primary neuronal cell culture}

\section{Treatment of coverslips}

For neuronal cell culture coverslips were incubated for minimum 2 hours in $1 \mathrm{M} \mathrm{HCl}$ (Roth, Germany) at $60^{\circ} \mathrm{C}$, before they were washed in acetone (Roth, Germany), $70 \%$ ethanol (Roth, Germany) and $99.9 \%$ ethanol (Roth, Germany) for 1 hour each. Coverslips were dried of the ethanol and autoclaved. Before use single coverslips were coated with $0.01 \%$ poly-L-lysine (Sigma-Aldrich, USA) or $0.04 \%$ poly(ethyleneimine) (Sigma-Aldrich, USA) solution for 12 hours. Coverslips were rinsed three times with water before plating the cells.

\section{Primary cell culture}

Primary cultures of hippocampal neurons were prepared according to the method of Dotti et al., (Dotti et al. 1988) with slight modifications. Hippocampi from E18 rat brains or PO mouse brains were washed with and collected in ice-cold calcium- and magnesium-free Hanks' balanced salts solution (HBSS). Cells were dissociated by 20 min incubation with $0.25 \%$ trypsin. Cells were washes three times with warm 
HBSS before dissociating the tissue by passing it through a needle 4-6 times. Dissociated cells were plated onto poly-D-lysine- or poly(ethyleneimine)- coated coverslips at a density of 60.000 cells $/ \mathrm{cm}^{2}$ in Dulbecco's modified Eagle's medium (DMEM) including $10 \%$ foetal calf serum (FCS), antibiotics $(100 \mathrm{U} / \mathrm{ml}$ penicillin, $100 \mu \mathrm{g} / \mathrm{ml}$ streptomycin), and $2 \mathrm{mM}$ glutamine. 12 hours after plating, the medium was exchanged for Neurobasal including $2 \%$ B27 (Life Technologies), antibiotics, and $0.5 \mathrm{mM}$ glutamine.

HEK293 cells were cultured in DMEM/F-12 (Invitrogen) medium supplemented with $10 \%$ FCS (PAA, Austria). Cells were plated at $10 \%$ initial confluency onto poly-Llysine coated coverslips. cDNA was transfected using Fugene HD (Roche, Switzerland) according to the supplier's protocol. Protein expression was allowed for 16-24 hours prior to conducting experiments. All cells were kept in a humidified $95 \%$ air, $5 \% \mathrm{CO}_{2}$ incubator.

\subsubsection{Transfection of DIV9 neurons}

Neurons were kept in Neurobasal including $2 \%$ B27 (Thermo Fischer, USA), antibiotics, and $0.5 \mathrm{mM}$ glutamine until DIV 9 before magnetofection using CombiMag (OZ BIOSCIENCES, France) according to method B of Buerli et al. (2007) with some modifications. $0.8 \mu \mathrm{g}$ cDNA were diluted in $150 \mu \mathrm{l}$ Neurobasal, while in a different reaction cup $7 \mu$ Lipofectamine 2000 (Invitrogen, USA) were mixed and incubated for $5 \mathrm{~min}$ at room temperature. Both formulations were combined and $1 \mu \mathrm{l}$ undiluted CombiMag (OZ BIOSCIENCES, France). The complex was incubated for 15 minutes at room temperature before it was distributed over four wells of a 24 well plate. The plate was incubated on a magnetic plate (IBA, Germany) at $37^{\circ} \mathrm{C}, 5 \% \mathrm{CO} 2$ for 25 minutes before replacing $80 \%$ of the culture medium. Expression was allowed for 72 hours.

\subsection{Immunocytochemistry}

Cells were fixed with $4 \%$ formaldehyde (Sigma-Aldrich, USA) in PBS, pH 7.4 for 20 minutes on ice. Permeabilisation was performed using $0.1 \%$ Triton X-100 (SigmaAldrich, USA) in PBS for $5 \mathrm{~min}$. After $10 \mathrm{~min}$ blocking with PBS-gelatin (SigmaAldrich, USA) (2 g/L), samples were incubated with primary antibodies (Table 2) over 
night at $4^{\circ} \mathrm{C}$ or for 2 hours at room temperature. After four washes with PBS-gelatin cells were incubated with secondary antibody as indicated (Table 3). Following two washes with PBS/gelatin and two washes with PBS, coverslips were mounted in Mowiol 4-88 (Aventis Pharma, Germany)

Table 2: List of primary antibodies

\begin{tabular}{|c|c|c|c|}
\hline Primary Antibody & Manufacturer & Order-No. & Dilution \\
\hline $\begin{array}{l}\text { Anti-mTOR antibody [Y391] } \\
\text { ab32028 }\end{array}$ & abcam, GB & ab32028 & 100 \\
\hline $\begin{array}{l}\text { Phospho-mTOR (Ser2448) } \\
\text { (49F9) Rabbit mAb (IHC } \\
\text { Specific) }\end{array}$ & $\begin{array}{l}\text { Cell Signaling } \\
\text { Technology, USA }\end{array}$ & \#2976 & 100 \\
\hline mTOR (7C10) Rabbit mAb & $\begin{array}{l}\text { Cell Signaling } \\
\text { Technology, USA }\end{array}$ & \#2983 & 200 \\
\hline $\begin{array}{l}\text { Phospho-mTOR (Ser2448) } \\
\text { (D9C2) XP® Rabbit mAb }\end{array}$ & $\begin{array}{l}\text { Cell Signaling } \\
\text { Technology, USA }\end{array}$ & \#5536 & 50 \\
\hline Akt (pan) (C67E7) Rabbit mAb & $\begin{array}{l}\text { Cell Signaling } \\
\text { Technology, USA }\end{array}$ & \#4691 & 200 \\
\hline $\begin{array}{l}\text { Phospho-Akt (Ser473) (D9E) } \\
\text { XP }^{\circledR} \text { Rabbit mAb }\end{array}$ & $\begin{array}{l}\text { Cell Signaling } \\
\text { Technology, USA }\end{array}$ & $\# 4060$ & 100 \\
\hline $\begin{array}{l}\text { GABA-A receptors y (guinea } \\
\text { pig) }\end{array}$ & $\begin{array}{l}\text { Synaptic Systems, } \\
\text { Germany }\end{array}$ & 224004 & 500 \\
\hline $\begin{array}{l}\text { Monoclonal Anti-c-Myc, } \\
\text { antibody produced in mouse }\end{array}$ & Sigma-Aldrich, USA & M4439-100UL & 1000 \\
\hline $\begin{array}{l}\text { HA.11 Clone 16B12 } \\
\text { Monoclonal Antibody }\end{array}$ & Covance, USA & MMS-101R & 1000 \\
\hline
\end{tabular}


Table 3: List of secondary antibodies

\begin{tabular}{|c|c|c|c|}
\hline Secondary Antibody & Manufacturer & Order-No. & Dilution \\
\hline $\begin{array}{l}\text { Cy3-conjugated AffiniPure } \\
\text { F(ab')2 Fragment Goat Anti- } \\
\text { Rabbit IgG F(ab')2 Fragment } \\
\text { Specific }\end{array}$ & $\begin{array}{l}\text { Jackson Immuno } \\
\text { Research, GB }\end{array}$ & $111-166-047$ & 1000 \\
\hline $\begin{array}{l}\text { Cy5-conjugated AffiniPure } \\
\text { F(ab')2 Fragment Goat Anti- } \\
\text { Rabbit IgG F(ab')2 Fragment } \\
\text { Specific }\end{array}$ & $\begin{array}{l}\text { Jackson Immuno } \\
\text { Research, GB }\end{array}$ & $111-176-047$ & 400 \\
\hline $\begin{array}{l}\text { AffiniPure Fab Fragment } \\
\text { Donkey Anti-Rabbit lgG }(\mathrm{H}+\mathrm{L})\end{array}$ & $\begin{array}{l}\text { Jackson Immuno } \\
\text { Research, GB }\end{array}$ & 711-007-003 & 500 \\
\hline $\begin{array}{l}\text { ATTO 532-conjugated } \\
\text { AffiniPure Fab Fragment } \\
\text { Donkey Anti-Rabbit IgG }(\mathrm{H}+\mathrm{L})\end{array}$ & $\begin{array}{l}\text { Jackson Immuno } \\
\text { Research, GB }\end{array}$ & 711-007-003 & 200 \\
\hline $\begin{array}{l}\text { ATTO 580Q--conjugated } \\
\text { AffiniPure Fab Fragment } \\
\text { Donkey Anti-Rabbit IgG }(\mathrm{H}+\mathrm{L})\end{array}$ & $\begin{array}{l}\text { Jackson Immuno } \\
\text { Research, GB }\end{array}$ & 711-007-003 & 500 \\
\hline $\begin{array}{l}\text { Alexa Fluor 647-conjugated } \\
\text { AffiniPure F(ab')2 Fragment } \\
\text { Donkey Anti-Guinea Pig IgG } \\
\text { F(ab')2 Fragment Specific }\end{array}$ & $\begin{array}{l}\text { Jackson Immuno } \\
\text { Research, GB }\end{array}$ & 706-606-148 & 500 \\
\hline $\begin{array}{l}\text { Alexa Fluor 647-conjugated } \\
\text { AffiniPure Goat Anti-Mouse } \\
\operatorname{lgG}(\mathrm{H}+\mathrm{L})\end{array}$ & $\begin{array}{l}\text { Jackson Immuno } \\
\text { Research, GB }\end{array}$ & $115-605-146$ & 500 \\
\hline
\end{tabular}

\subsection{Antibody labelling with amine-reactive ATTO-labels}

Antibody labeling was performed according to the protocol provided by ATTO-TEC with slight changes. $200 \mu \mathrm{g}$ glycerin-free monovalent Fabs fragment (Table 2) were diluted in $200 \mu \mathrm{l} 10 \mathrm{mM}$ sodium bicarbonate buffer $(\mathrm{pH}=8.3)$. ATTO 532-NHS ester (ATTO-TEC, Germany) was diluted to $10 \mathrm{mM}$ in anhydrous, amine-free DMSO. ATTO 580Q-NHS ester was diluted to $10 \mathrm{mM}$ in anhydrous, amine-free DMF. A 3- 
fold molar excess of ATTO dyes was combined with the antibody solution and incubated for $30 \mathrm{~min}$ protected from light at room temperature.

Following, the Fab fragments were separated from unbound dye by gel filtration over an Econo-Pac 10 DG Disposable Chromatography Column (Bio-RAD, USA). The efflux was collected in $1 \mathrm{ml}$ fractions and the absorption of every fraction was measured at $280 \mathrm{~nm}$. Antibody-containing fractions were combined and concentrated over Amicon ultra centrifugal filter $10 \mathrm{~K}$ (Millipore, Germany). A UV-VIS spectrum of the concentrated antibody solution was obtained and the degree of labelling was determined.

\subsection{PCR Cloning}

The P1 isoform (Prior et al., 1992) of rat pEGFP-gephyrin and rat pmCherry-gephyrin was obtained from RJ Harvey, University College London. The S200A, S268A and S270A mutants were generated by site directed mutagenesis using QuickChange XL Site-Directed Mutagenesis Kit (Stratagene, USA) according to the manufacturer instructions using specific primers (Table 4).

Table 4: Gephyrin mutant primers

\begin{tabular}{|l|l|}
\hline $\begin{array}{l}\text { Primer } \\
\text { Name }\end{array}$ & Sequence $\left(5^{\prime}-3^{\prime}\right)$ \\
\hline S200A fwd & CTTTCTCCACCTCCTACAACTGCGCCACATAAGCAGACAGAAGAC \\
\hline S200A rev & GTCTTCTGTCTGCTTATGTGGCGCAGTTGTAGGAGGTGGAGAAAG \\
\hline S268A fwd & CTTAGCACTACTCCTGCAGAGTCGCCCCGTGCC \\
\hline S268A rev & GGCACGGGGCGACTCTGCAGGAGTAGTGCTAAG \\
\hline S270A fwd & GCACTACTCCTTCAGAGGCGCCCCGTGCCCAGGCTAC \\
\hline S270A rev & GTAGCCTGGGCACGGGGCGCCTCTGAAGGAGTAGTGC \\
\hline
\end{tabular}

The cDNA of $p R K 5$ myc-CB2 $2_{S H 3}$ was obtained from the lab of RJ Harvey. HA-tagged neuroligin 2 (HA-NL2 in pcDNA3) (S. Jamain, Faculté de Médecine-Hôpital Henri Mondor, Créteil, France), gephyrin clustering mutants RER EGFP-gephyrin and $4 \times R$ EGFP-gephyrin (described in Saiyed et al., 2007) were kindly provided by $T$. 
Papadopoulos (Max-Planck-Institut für Experimentelle Medizin, Göttingen). The cDNA of EGFP-mTOR and mTOR-EGFP was obtained from Stanley Botchway (Central Laser Facility, STFC, Rutherford Appleton Laboratory, Research Complex at Harwell, Didcot, Oxon OX110QX, UK.)

\subsection{Cloning of C-terminally labelled Gephyrin constructs}

The sequence of rat Gephyrin was amplified by PCR from $p E G F P$-gephyrin using the primer containing restriction sites for Kpn1 and Xma1 below.

Table 5: C-terminally labelled Gephyrin constructs

\begin{tabular}{|l|l|}
\hline Primer name & Primer sequence (5’ - 3') \\
\hline GPHN-Kpn1-fwd & 5'-TAAGCAGGTACCATGGCGACCGAGGGAATGAT-3' \\
\hline GPHN-Xma1-rev & 5'-TGCTTACCCGGGATAGCCGTCCGATGACCATGAC-3' \\
\hline
\end{tabular}

The PCR product and acceptor vectors, pmTurquoise-N1 and pEYFP-N1 (Clonetec), were digested with Xma1 and Kpn1 and ligated using the Rapid DNA Dephos and Ligation Kit (Roche, Switzerland) according to the manufacturer instructions. The resulting fusion constructs, Gephyrin-Cerulean and Gephyrin-EYFP, were checked by sequencing.

\subsection{Microscopy}

Confocal images were taken on a FluoView1000 (Olympus, Japan) equipped with a 60×NA 1.35 UPLS-Apo objective (Olympus, Japan). mTurquoise, EGFP and EYFP were excited with the 456 and $515 \mathrm{~nm}$ lines of an Argon laser (company, country); the emission was separated by an 458 dichroic mirror. EGFP and mCherry and were excited with a $559 \mathrm{~nm}$ line of laser diode (NTT electronics, Japan); Alexa647 and Cy5 were excited with the $640 \mathrm{~nm}$ line of laser diode (Olympus, Japan), respectively.

Fluorescence lifetime images were recorded using a pulsed laser diodes of $440 \mathrm{~nm}$, $485 \mathrm{~nm}$ and $532 \mathrm{~nm}$ driven by a Sepia II unit (Picoquant $\mathrm{GmbH}$, Berlin Germany) at a repetition rate of $40 \mathrm{MHz}$ (Picoquant $\mathrm{GmbH}$, Berlin, Germany). Fluorescence was 
reflected on a PDM series single photon avalanche diode (Picoquant $\mathrm{GmbH}$, Germany).

\subsection{Image Analysis, Quantification and Representation}

Images in PT3 format were converted into ICS format (Dean et al., 1990) using PTC2ICS software (Geert van den Bogaart). ICS files were analysed using TRI2 software version 2.8.5.1 (PR Barber, RJ Locke, RJ Edens, SM AmeerBerg, B Vojnovic, GP Pierce, and M Rowley). The images were thresholded by intensity in TRI2 before all counts represented in phasor plot. Distributions of monoexponential lifetimes were derived from Phasor plots. The distributions were normalized to unity averaged and fitted to Gaussian distributions in GraphPad prism version 5.01.

\section{Statistics}

To determine difference between conditions, the standard error of the mean was used to calculate p-values. Due to the fact that nearly every observed difference between conditions of an experiment was significant with a value of $p<0.0001, p$ values are not represented in the figures. The use of the standard error of the mean was indicated, the lifetime of a fluorophore is not a subject of population variance. Nevertheless, standard deviations are given in the text to refer to the width of a given distribution.

\section{Image representation}

To generate FRET pictures the inverted look up table was applied to the lifetime images in a before set lifetime range. The pictures were exported from TRI2 in TIFF format and converted into RGB format using Image J software (version 1.47, Wayne Rasband). The RGB format was converted and into HSB stack in which the B layer was replaced by the intensity image of that same picture. The HSB stack was then converted back into RGB. 


\section{Results}

\subsection{Establishment of antibody-based phosphorylation assays}

Posttranslational modifications, like phosphorylation or acetylation, are fast signalling events that sustain or change the signalling properties of molecules within the cell. Hence, they can be used as markers for signalling states.

mTOR is phosphorylated at $\mathbf{S} 2448$ when the cell is in a state of growth and glucose, amino acids and growth factors are in sufficient supply. mTOR sustains translation by being assembled in $\mathrm{mTORC} 1$, which phosphorylates S6K1/2 and E4-BP1 to initiate mRNA translation. Rapamycin or insufficient supply of amino acids, glucose or growth factors inhibits mTORC1 and terminates mRNA translation (Laplante and Sabatini et al., 2012).

Phosphorylation-specific antibodies are indispensable tools in biochemistry to assess the phosphorylation states of proteins. Standard techniques to detect phosphorylation levels include lysis of large number of cells which are then subjected to gel electrophoresis, blotting and subsequent detection with antibodies. This technique can be used to roughly estimate the average phosphorylation of a given protein. All cell type-specific or cell state-specific aspects of phosphorylation events like oscillations are averaged.

Most signalling molecules including mTOR and Akt show distinct cellular localisations depending to their signalling state. It is therefore necessary to connect the phosphorylation status with spatial information to fully comprehend their function.

Other approaches utilise phosphorylation-specific binding motives like $\mathrm{SH} 3$ domains coupled to fluorescent molecules to detected changes in phosphorylation states. They can be used in live cells to analyse dynamic signalling processes. Nevertheless, these domains introduce functional entities that have the capacity to interfere with the events that they are supposed to report on.

A more quantitative way to measure the phosphorylation states are immunofluorescence-based ratiometric assays. To this end, two antibodies are used to quantify the total protein and the phospho-epitope. The relative phosphorylation level is obtained from determining the ratio of fluorescence intensity of the individual labels. The advantage of this method is that it can be used to analyse endogenous 
signalling events without the use of exogenous reporter molecules. The major disadvantage of this type of assay is that it depends on the quality of the antibodies. The ratio may also amplify unspecific signals, veiling physiological effects.

One way to manage the disadvantages of antibody-based assays is the use of FRET as a "clean-up" method. The energy transfer process between donor and acceptor molecules rely on the extremely short-ranged interactions of fluorophore dipoles. The efficiency of energy transfer drops from "all" to "nothing" within a few nanometres. If FRET can be measured between antibodies directed against adjacent epitopes, it is highly likely that both antibodies have bound their specific targets. Hence; FRET can be used to evaluate the specificity of an antibody. Given the distance-dependence of FRET, binding to unspecific targets, would not generate a FRET signal. In fluorescence lifetime microscopy, unspecific binding ads to the non-FRET portion which has the potential to dilute the FRET signal but will not generate false-positive FRET.

\subsubsection{Antibody choices}

Commercially available antibodies were selected on the basis of:

1. Applicability in immunofluorescence experiments

2. Location of the epitope in the amino acid sequence

3. Target and host species

4. Popularity (number of application-relevant publications)

The C-terminal portion of mTOR was chosen as target epitope because it appeared most likely to come in close proximity to the $\$ 2448$ epitope. The criteria listed above were only met by two antibodies: anti-mTOR antibody [Y391] from abcam (Cambridge, England) and mTOR (7C10) Rabbit mAb from Cell Signaling Technologies (Cambridge, England). Both antibodies were used for all following experiments. They will be referred to as "abcam" and "7C10" hereafter.

Amongst the antibodies directed against phosphorylated S2448, two antibodies were chosen: "phospho-mTOR (S2448) (49F9) Rabbit mAb (IHC specific)" and "PhosphomTOR (S2448) (D9C2) XP Rabbit mAb", hereafter referred to as "IHC-specific" and "XP". 
To compare the staining patterns among each other and across spiecies all 4 antibodies were tested on rat embryonic fibroblasts (REF-52) and human embryonic kidney cells (HEK293) using the dilutions recommended by the manufacturer (Figure 6). The staining patterns could be divided into two categories. 1: unstructured cytoplasmic staining with strong nuclear or perinuclear signal or 2: prominent granular structures throughout the cytoplasm but no nuclear signal. The abcam and the IHC-specific antibody fitted the first category. The 7C10 and the XP antibody fitted the second category.

A
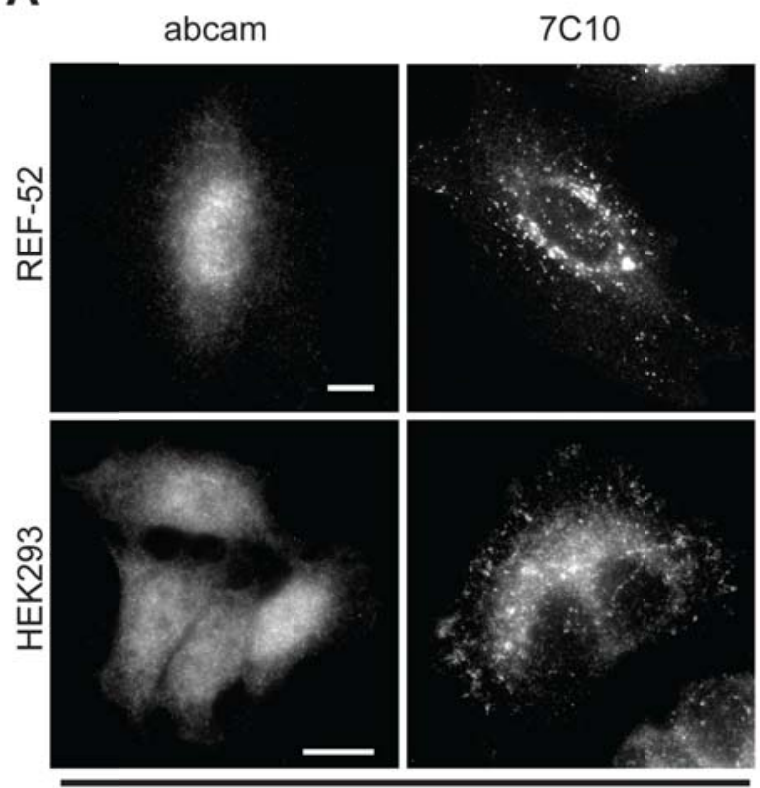

total mTOR

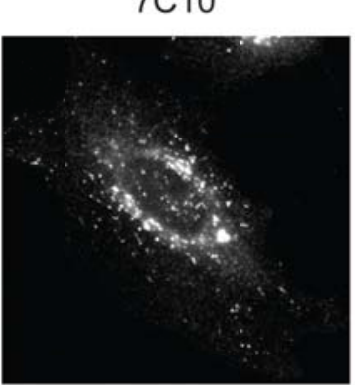

B
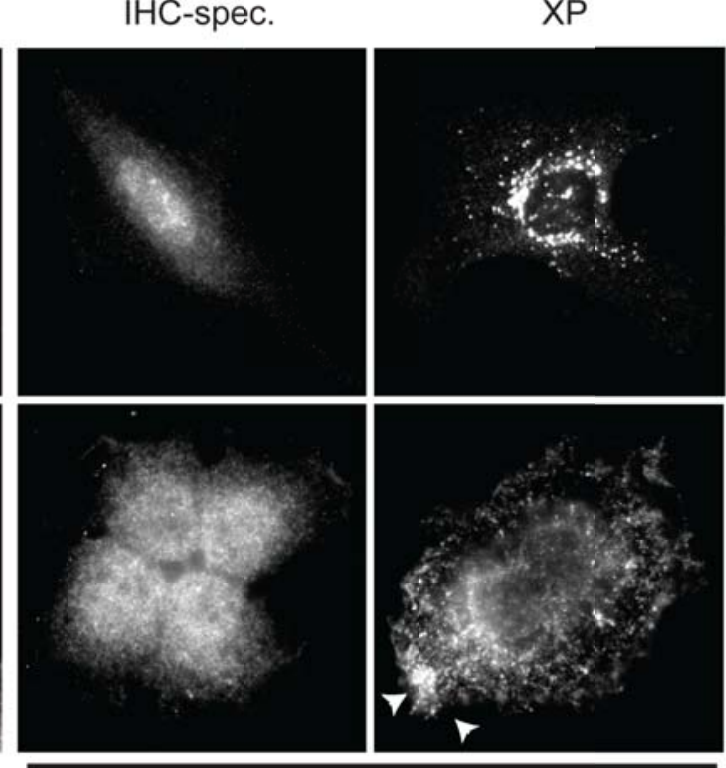

pS2448-mTOR

Figure 6: Antibody stainings of total and phosphorylated mTOR

A: Immunofluorescence staining for total mTOR were carried out with two different antibodies, abcam and 7C10 on REF52 cells (rat) (top row) (grascale) and HEK293 cells (human) (bottom row) (grascale) to compare staining patterns between antibodies and across species. The abcam antibody detects epitopes in the nucleus and the cytoplasm in REF52 and HEK293 cells. The 7C10 antibody detects epitopes primarily on granular structures throughout the cytoplasm in REF52 and in HEK293 cells. B: Immunofluorescence staining for mTOR phosphorylated at S2448. Two antibodies, IHC-spec and XP, were compared. The IHC-spec recognises epitopes in the nucleus and the cytoplasm while the XP recognises epitopes on granular structures in the cytoplasm and a nuclear staining is missing. The granular structures seem concentrated in the lamellopod in the HEK293 cell (white arrows). Scale bar: $20 \mu \mathrm{m}$ 
In 2012, when these experiments were conducted, mTOR was considered to be localised in the cytoplasm. The first reports about a lysosomal localization of mTORC1 were published in 2010 (Sancak et al. 2010) but only later in 2013 a recruitment of mTORC1 components to endosomal or lysosomal membranes was confirmed (Zhou et al. 2013). The cytoplasmic staining pattern was therefore preferred over the granular staining pattern.

Several components of mTORC1 including mTOR have been reported to have nuclear localization (Kim and Kahn 1997; Kim and Chen 2000; Zhang et al., 2002; Kikani et al., 2005; Liang and Di Cristofano, 2005; Rosner et al., 2007; Rosner and Hengstschläger, 2008; Rosner and Hengstschläger, 2012). These two observations lead to the conclusion that the diffuse cytoplasmic and bright nuclear staining patterns observed with the abcam and the IHC-specific antibodies had to be correct. Both antibodies were therefore used in the following FRET experiments.

\subsubsection{Assay considerations}

A phosphorylation assay is supposed to report on the portion of total protein that is phosphorylated. In donor based FRET measurements the total protein should be labeld the donor fluorophore because it is likely to be constant. The phosphorylated epitope should be labelled the acceptor because it may vary dynamically which would lead to high FRET efficiencies at high phosphorylation levels and low FRET efficiencies at low phosphorylation levels. If the phospho-epitope was labeld donor, the signal may be lost at low phosphorylation levels which would render the assay unmeasurable.

The chosen antibodies were all raised in rabbit. Consequently, the FRET assay required a sequential application of the primary antibodies. It was therefore to be answered which antibody should come first. Phosphorylation-specific antibodies usually have lower affinities than antibodies directed against unphosphorylated targets. Therefore the total mTOR antibody was applied first because it seemed more likely to endure repeated washing. Experiments in which the pS2448 was used first confirmed this assumption (data not shown).

With two antibodies from the same spiecies a certain amount of cross-reactivity had to be taken into considderation. It was anticipated that the Cy3- and Cy5-conjugated 
$\mathrm{F}(\mathrm{ab})_{2}$ fragments would cross-react, since they are both directed against rabbit. The cross-contamination was therefore determined by incubating the Cy3-decorated total mTOR antibody (abcam) with Cy5-conjugated $\mathrm{F}(\mathrm{ab})_{2}$ fragments. This was used as cross-reactivity control and is called 'half assay' in all following figures (Figure 7).

\section{A}

'Half Assay'

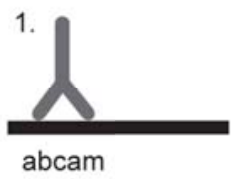

2.

$\mathrm{F}(\mathrm{ab})_{2}-\mathrm{Cy} 3$

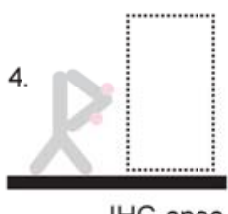

IHC-spec
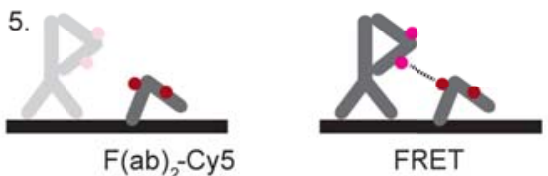

FRET

B

'Full Assay'

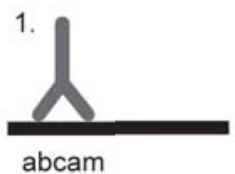

2.

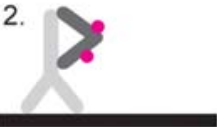

$\mathrm{F}(\mathrm{ab})_{2}-\mathrm{Cy} 3$

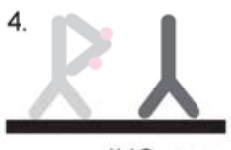

IHC-spec.

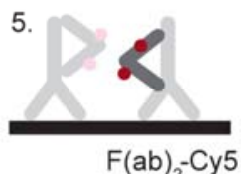

$\mathrm{F}(\mathrm{ab})_{2}-\mathrm{Cy} 5$

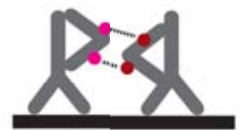

FRET

Figure 7: Schematic for staining procedure without blocking antibodies

A: The cross-reactivity between the Cy5-conjugated $F(a b)_{2}$ fragments and the Cy3-decorated abcam antibody was determined by leaving out the IHC-specific antibody (dotted box). The measured cross-reactivity was used as reference to calculate FRET. B: The 'Full Assay' comprises two primary antibodies and two labelled secondary antibodies.

The FRET pair that was used in this assay is Cy3 and Cy5. They are a commonly used as they exhibit a high Förster distance which allow for high FRET efficiencies.

The assay was conducted in REF-52 cells according to the considderations above (Figure 8). Fluorescence lifetime images of Cy3 were recorded, fitted pixel-by-pixel and are displayed in false-colour representation. For the cumulated lifetime distributions a minimum of five FLIM measurements per condition were normalised to unity, averaged and fitted to a Gaussian distribution. The FRET efficiencies were calculated and displayed in the same way. The total mTOR, when labelled with $\mathrm{F}(\mathrm{ab})_{2}$-Cy3, had a mean lifetime of $1.64 \mathrm{~ns}$ (SD: $0.096 \mathrm{~ns}$ ). The cross-reactivity control termed "Half assay" shows a mean lifetime of 1.54 ns (SD: $0.17 \mathrm{~ns}$ ). The "Full assay" shows a lifetime of 1.39 ns (SD: 0.13 ns). The reduction of the Cy3 lifetime by 
cross-reactivity with Cy5-conjugated $\mathrm{F}(\mathrm{ab})_{2}$-fragments is 100 ps or $6.27 \%$ FRET. Cross-reactivity creates a significant offset in this assay. Consequently, the crossreactivity control was used as reference point in order to calculate the relative FRET efficiency. The relative increase in FRET efficiencies by the interaction of the two labeled mTOR epitopes was measured $9.7 \%$. The highest FRET efficiencies were observed in the nucleus and in the cytoplasm, reaching up to $25 \%$. The prominent bright puncta that are observed throughout the cytoplasm in the intensity image did not show any FRET. 
A
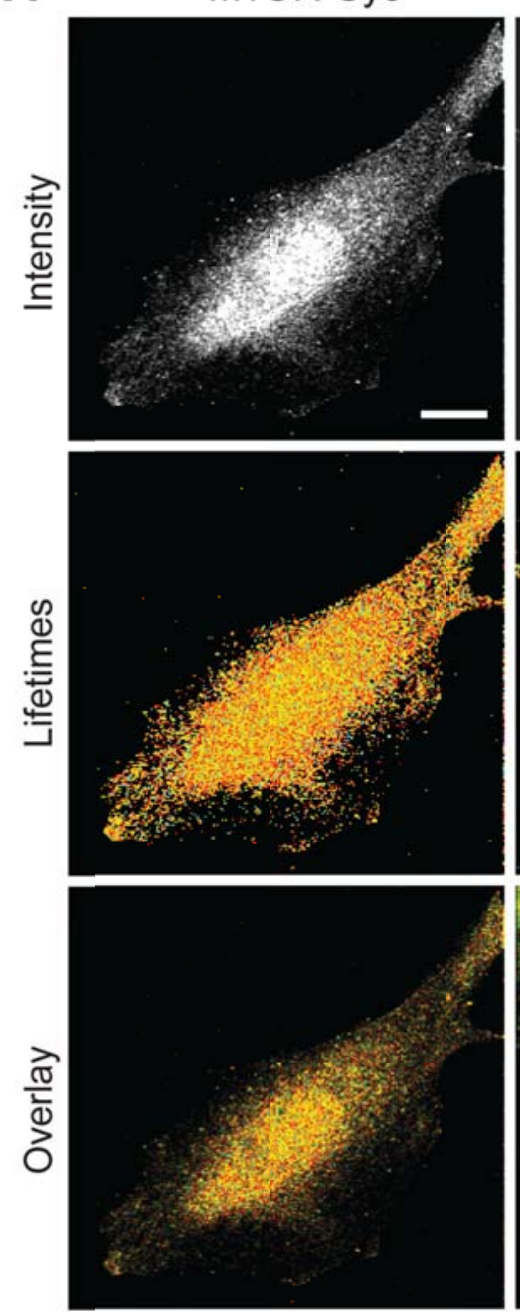

B

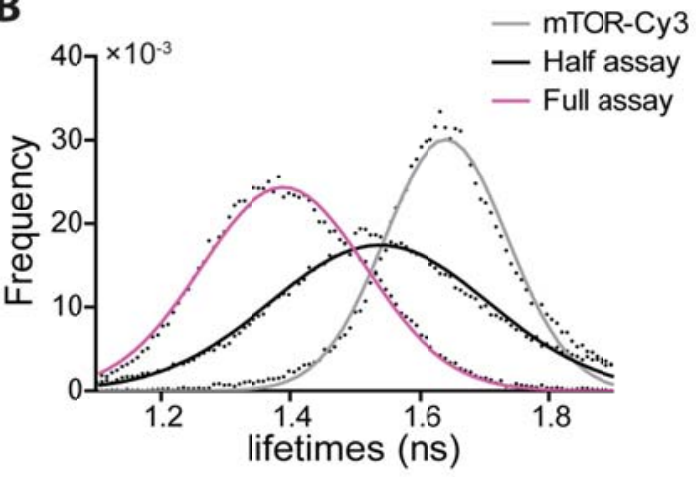

Half assay
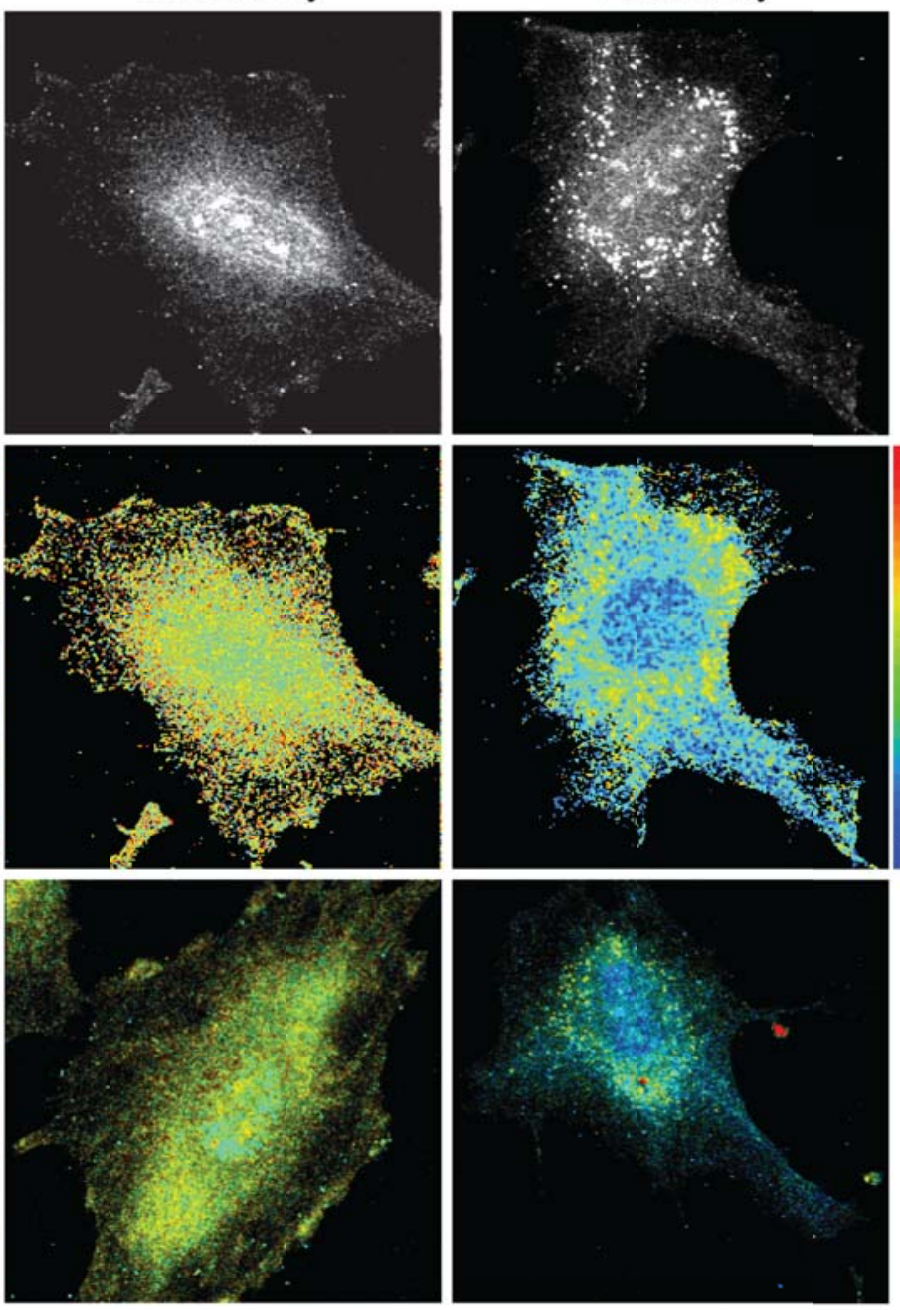

Full assay

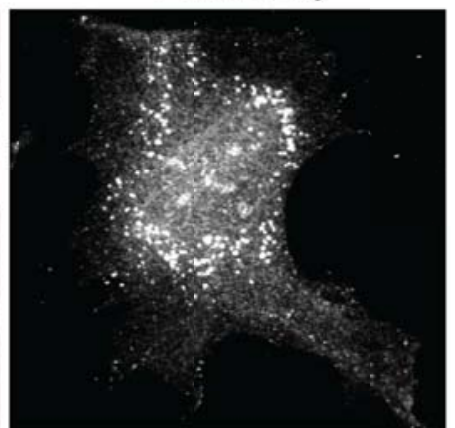

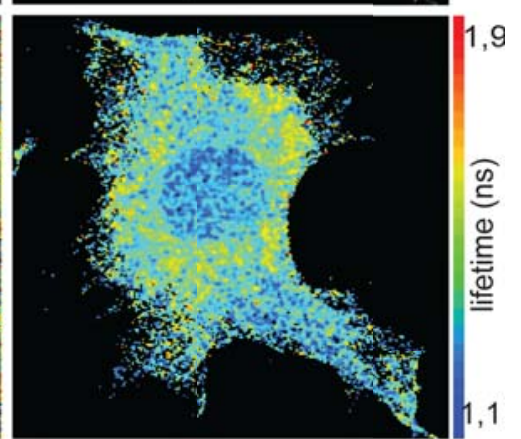

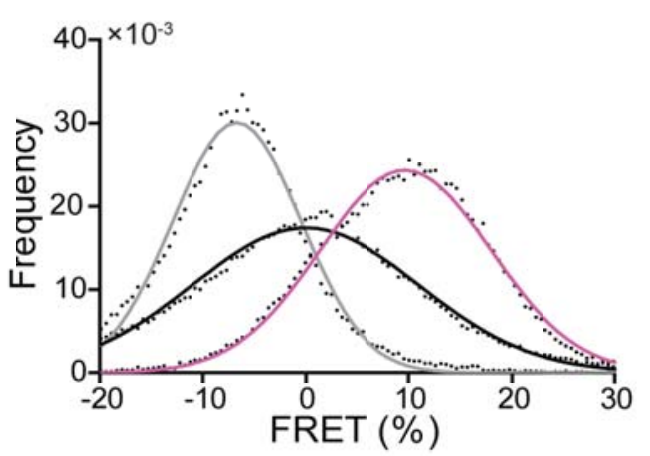

Figure 8: Staining of mTOR phosphorylation without blocking antibodies

A: Shown are representative intensity images stained with Cy3, 'Half assay' looking at cross-reactivity and 'Full assay' looking at phosphorylated mTOR (grayscale) (top row). Corresponding lifetime images are displayed in false colour (middle row).The overlay of intensity and lifetime images shows the relative contribution of single pixels (bottom row). Scale bar: $20 \mu \mathrm{m}$. B: Averaged lifetime distributions and FRET efficiency distributions calculated from the lifetime distributions per condition are shown, $n=5$. 
The cross-reactivity between the secondary antibodies was quite high and also varied over a broad range. It was therefore tested if monovalent Fab fragments would impede the binding of Cy5-conjugated $\mathrm{F}(\mathrm{ab})_{2}$ fragments by reducing the number of accessible epitopes (Figure 9).

\section{A}

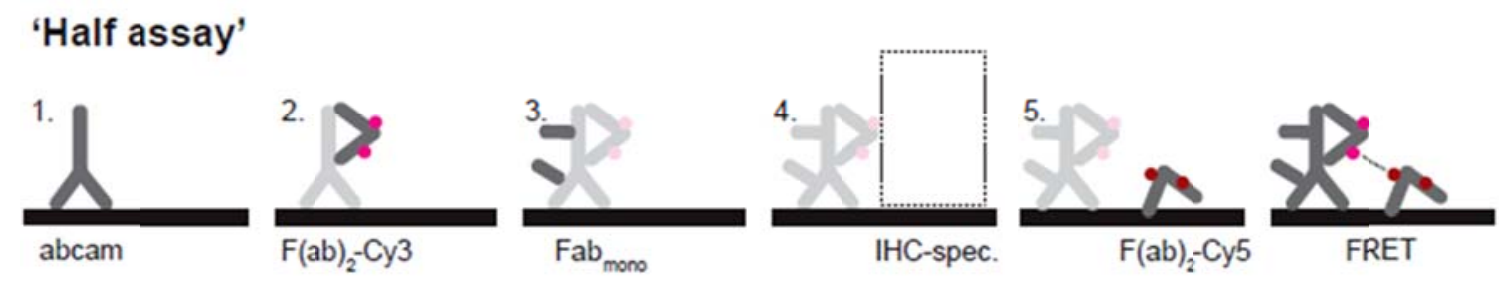

B

'Full assay'

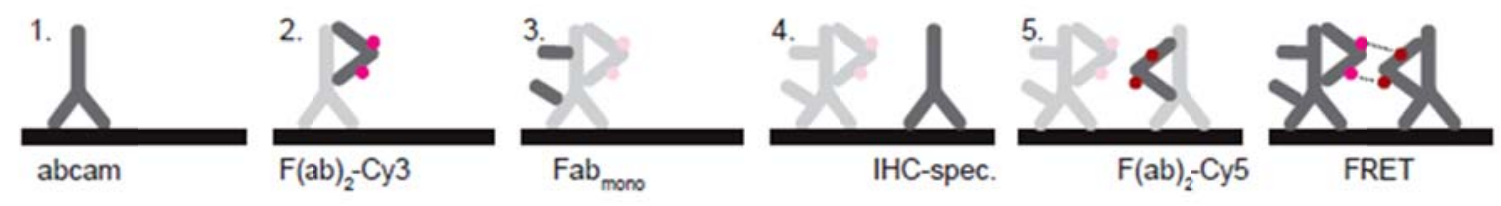

Figure 9: Schematic for staining procedure with blocking antibodies

A: Cross-reactivity was determined by leaving out the 'IHC-specific' antibody (dotted box). The measured cross-reactivity is used it as reference to calculate FRET. B: The 'Full assay' comprises two primary antibodies, a blocking Fab fragment and two labelled secondary antibodies in the sequence depicted above.

With the additional blocking step, the staining pattern of the mTOR antibody was identical to that observed in experiments without Fab-blocking. The lifetime of the cross-reactivity control is $1.57 \mathrm{~ns}$ (SD: $0.09 \mathrm{~ns}$ ). The full assay shows a lifetime of 1.34 ns (SD: 0.1 ns) which corresponds to 11.4 \% FRET. Monovalent Fab fragments increased the mean FRET efficiencies and decreased the standard deviation by $50 \%$. Blocking was a useful improvement and was applied in all following measurements that used conjugated $\mathrm{F}(\mathrm{ab})_{2}$ fragments (Figure 10). 
A
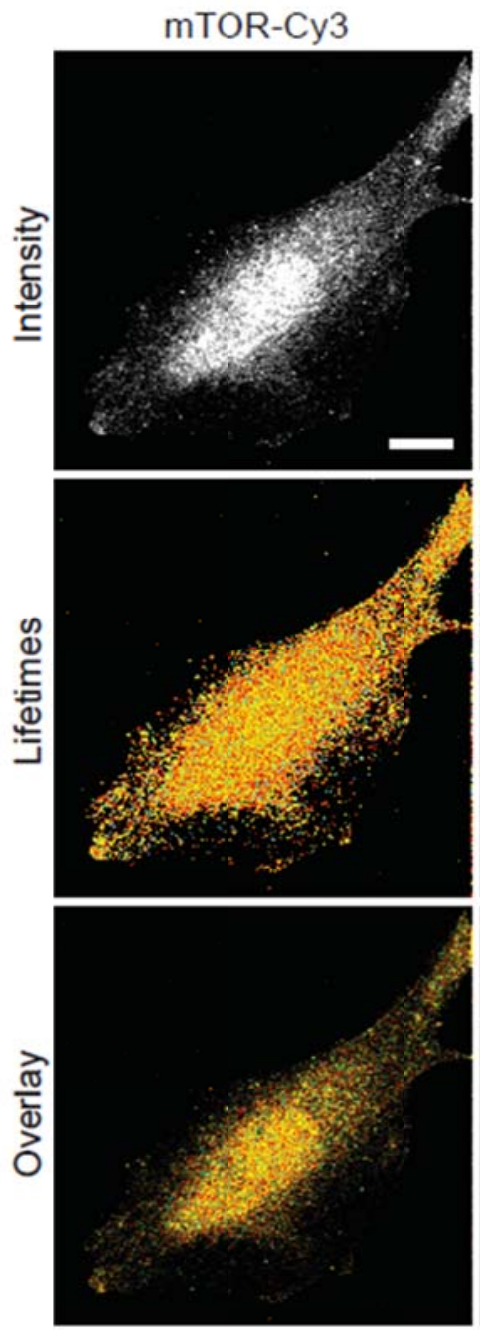

Half assay
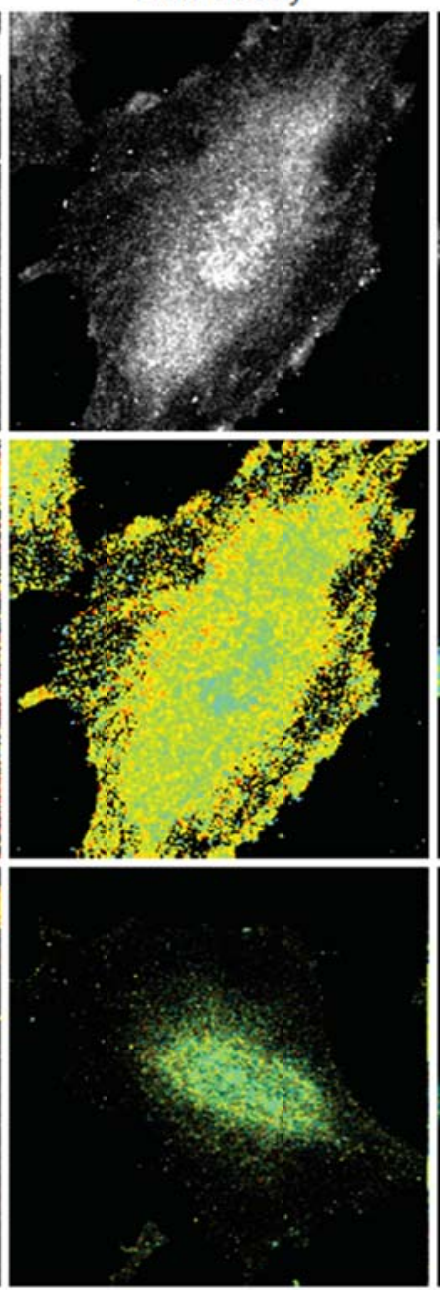

Full assay
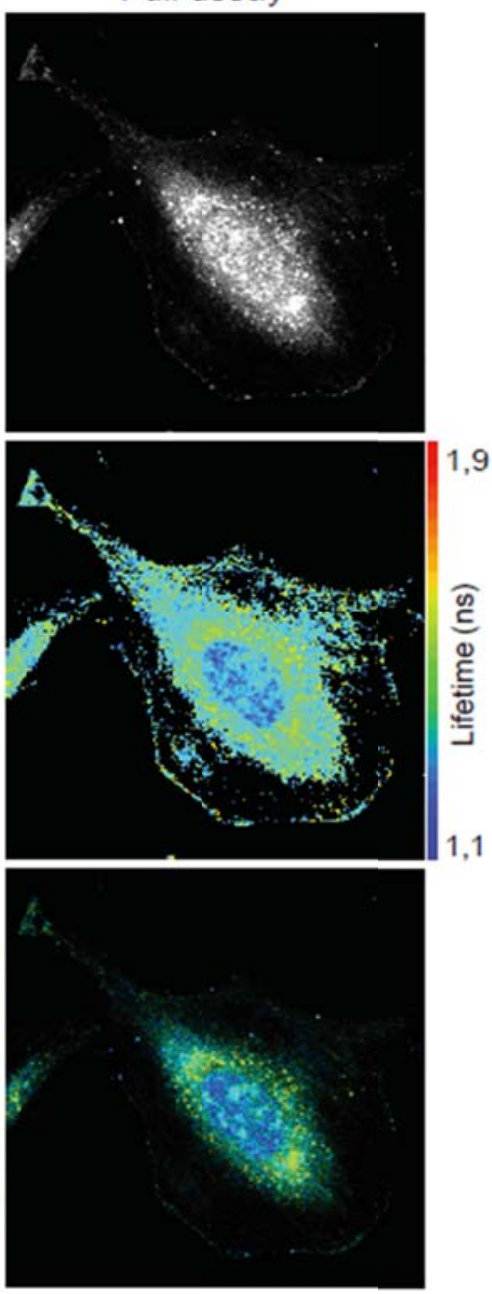

B
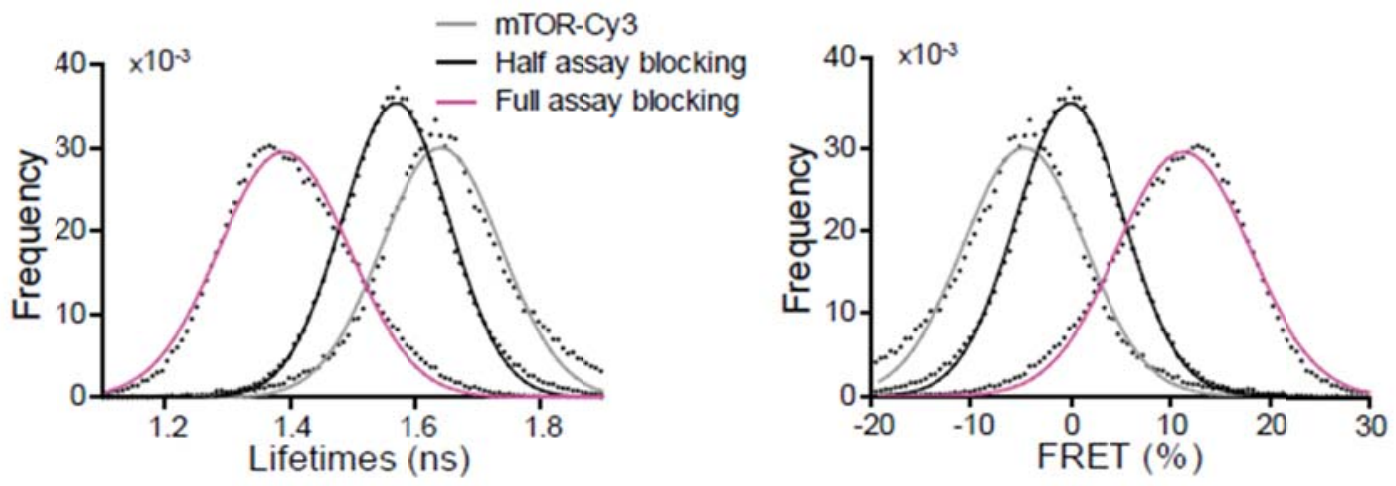

Figure 10: mTOR phosphorylation assay with blocking antibodies

A: Intensity images of mTOR stained Cy3, 'Half assay' looking at cross-reactivity and 'Full assay' looking at phosphorylated mTOR (Top row). Corresponding lifetime images (false colour) (middle row), overlay of lifetime and intensity images (bottom row) show relative contributions of each pixel. Scale bar: $20 \mu \mathrm{m}$. B: Averaged lifetime distributions and calculated FRET efficiency distributions are shown, $\mathrm{n}=5$. 


\subsubsection{FRET optimisation}

FRET assays need to be optimized in respect to application and measurement requirements. We use TCSPC FLIM, a technique with the highest achievable accuracy and sensitivity among all FRET techniques. The high precision is achieved by a high number of collected photons, which can be provided by long recording times or high photon count rates. Therefore, fluorophores photostability and brightness become important characteristics.

Cy3 has moderate relative brightness but low photostability, which limits its application for TCSPC FLIM experiments. We therefore searched for alternatives with a similar emission profile.

ATTO 532 has similar extinction coefficient at $532 \mathrm{~nm}$ but a higher photostability than Cy3 and most importantly a 6-fold higher quantum yield. ATTO 532 was therefore considered as a potential substitute for $\mathrm{Cy} 3$ which was tested subsequently by direct comparison (Figure 11).

ATTO 532's longer lifetime of 3.2 ns (conjugated) is beneficial for FRET as it increases the dynamic range of the measurement. The same relative increase in FRET efficiencies of $10 \%$ results a larger relative changes of the lifetimes.

Under half assay conditions, the lifetime of ATTO 532 is $2.8 \mathrm{~ns}$ (SD: $0.21 \mathrm{~ns})$. The full assay shows a lifetime of $2.5 \mathrm{~ns}$, which corresponds to $11.91 \%$ FRET (SD: $7.3 \%$ ). In comparison, the Cy3/Cy5-based mTOR assay shows a FRET efficiency of only 8.8 $\%$ (SD: $9.6 \%$ ). The increased FRET efficiency and the lower SD are desirable improvements for sensitive FRET assays. 
A

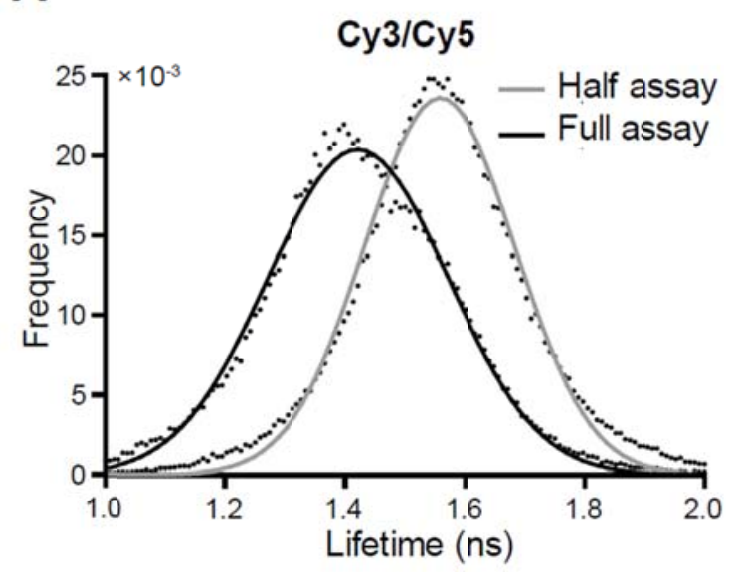

B

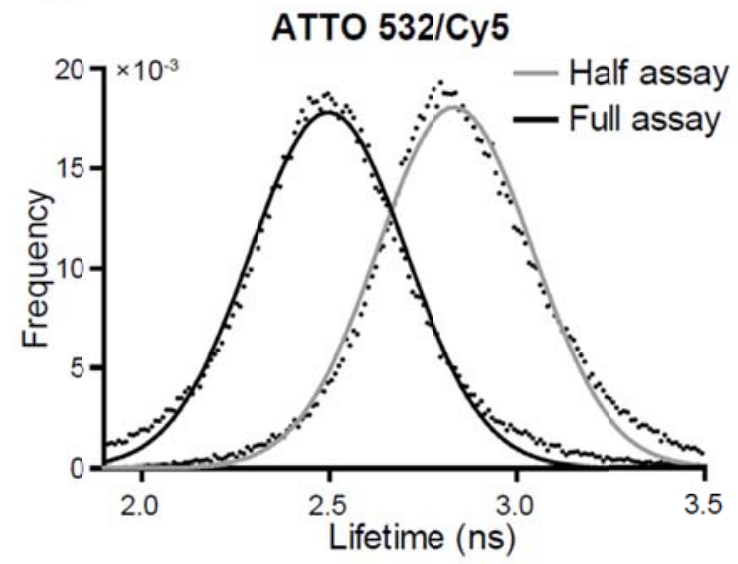

C Cy3/Cy5 vs. ATTO 532/Cy5

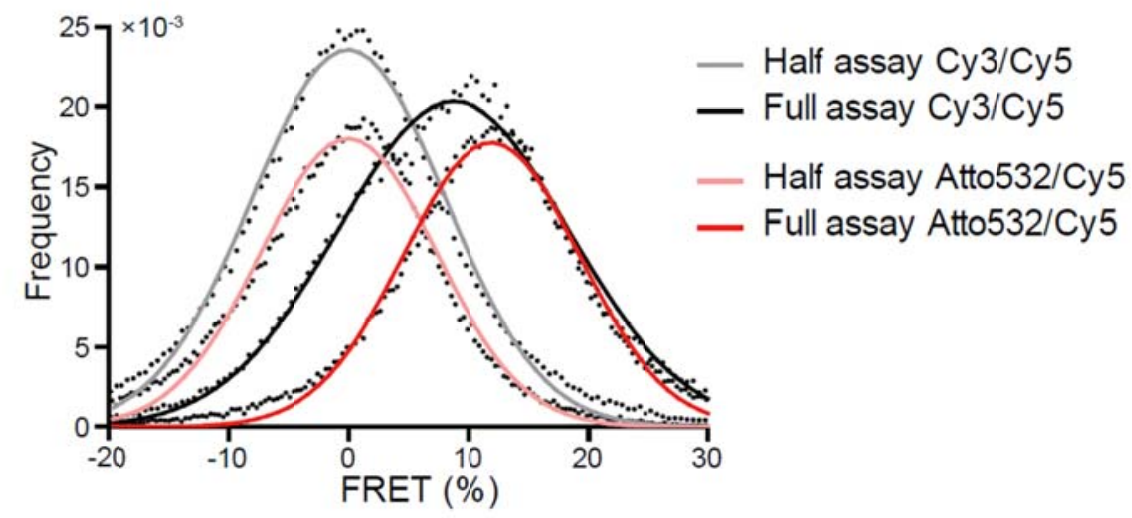

Figure 11: Exchanging Cy3 for ATTO 532 is beneficial for FRET

A: REF-52 cells were stained with mTOR-Cy3 and pS2448-Cy5. The fluorescence lifetimes of Cy3 was measured in absence (Half assay) and presence of the pS2448 antibody (Full assay). All graphs were normalised and the average lifetime distribution displayed, $n=10$ cells.

B: REF-52 cells were stained with mTOR-ATTO 532 and pS2448-Cy5. The fluorescence lifetimes of ATTO 532 was measured in absence (Half assay) and presence of the pS2448 antibody (Full assay). All graphs were normalised and the average lifetime distribution displayed, $n=10$ cells. C: FRET efficiency distributions calculated from the fluorescence lifetimes in $A$ and $B$.

The Förster distance of a donor and acceptor pair is defined as the distance at which the energy transfer efficiency is $50 \%$. It depends on the overlap integral of the emission spectrum of the donor and the absorption spectrum of the acceptor. The higher the Förster distance, the more likely it is to measure higher FRET efficiencies. The Förster distance between Cy3 and Cy5 is $54 \AA$,while the Förster distance between ATTO 532 and ATTO $580 Q$ is $67 \AA$. 
It was therefore tested if replacing ATTO $580 Q$ for Cy5 is beneficial. ATTO $580 Q$ is a fluorescence quencher that will absorb energy without emitting photons. This allows recording of the donor fluorescence over a wider spectral range or to re-use the acceptor spectral window for detecting cellular markers. The combination of FRET assays with molecular markers for biological structures adds substantial information for understanding localized signalling events.

ATTO 532 with Cy5 shows a mean lifetime of 2.9 ns (SD: 0.17 ns) under half assay conditions and 2.46 ns (SD: $0.15 \mathrm{~ns}$ ) under full assay conditions. The lifetime difference of 430 ps corresponds to a FRET efficiency of $14.73 \%$ (SD: $5.24 \%$ ). When combined with ATTO $580 Q$ the lifetime of ATTO 532 was measured as $2.77 \mathrm{~ns}$ (SD: $0.15 \mathrm{~ns}$ ) under half assay conditions and $2.2 \mathrm{~ns}$ (SD: $0.15 \mathrm{~ns}$ ) under full assay conditions. The lifetime difference of 570 ps corresponds to a FRET efficiency of $20.66 \%$ (SD: $5.26 \%$ ) which represents an increase of nearly $6 \%$ FRET. This data clearly demonstrates the benefit of a larger Förster distance (Figure 12). 

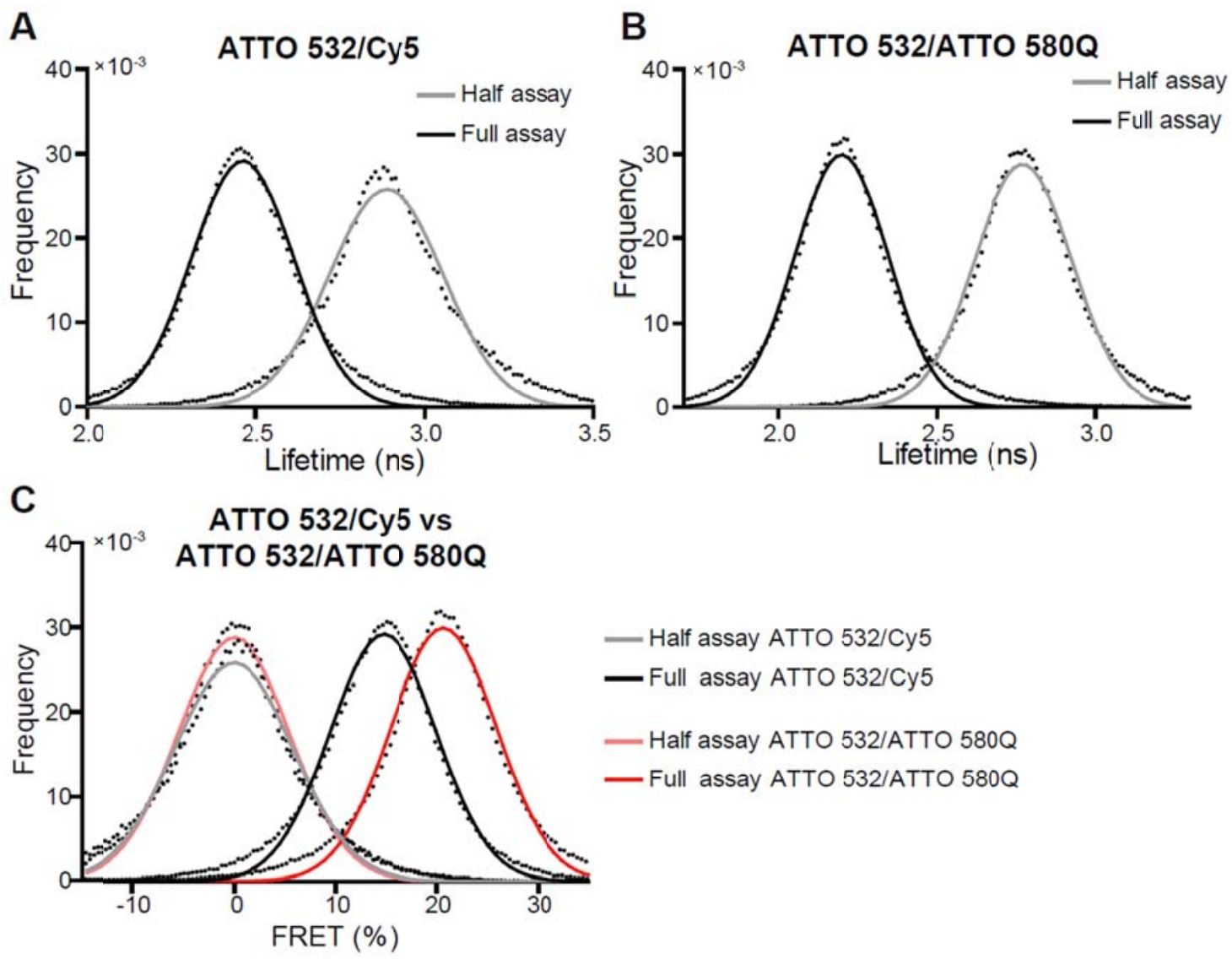

Figure 12: ATTO 580Q improves FRET compared with Cy5

A: REF-52 cells were stained with mTOR-ATTO 532 and pS2448-Cy5. The fluorescence lifetimes of ATTO 532 was measured in absence (Half assay) and presence of the pS2448 antibody (Full assay). All graphs were normalised and the average lifetime distribution displayed, $n=10$ cells. B: REF- 52 cells were stained with mTOR-ATTO 532 and pS2448-ATTO $580 Q$. The fluorescence lifetimes of ATTO 532 was measured in absence (Half assay) and presence of the pS2448 antibody (Full assay). All graphs were normalised and the average lifetime distribution displayed, $n=10$ cells. C: Comparison of the FRET efficiency distributions calculated in A and B. 


\subsubsection{Physiological validation}

Rapamycin and nutrient starvation are potent inhibitors of mTORC1 activity. In turn, stimulation with nutrients or growth factors activates mTORC1. These dynamic changes in mTORC1 activity are reflected by the S6K1/2-dependent phosphorylation at S2448 of mTOR (Laplante and Sabatini, 2014).

REF-52 cells were treated with rapamycin for 1, 12 or 24 hours and phosphorylation of S2448 was measured (Figure 13). Rapamycin did not change the FRET efficiency compared to unstimulated cells, although from the literature, it is known that rapamycin reduces the phosphorylation of S2448 already after $10 \mathrm{~min}$.

Cells were also re-stimulated with FCS after starvation. Starvation conditions were created by culturing the cells for 18 hours in medium supplemented with $0.1 \%$ FCS as opposed to $10 \%$ FCS under normal cell culture conditions. After 18 hours of starvation, the cells were stimulated for 30 min with medium containing $10 \%$ FCS. Figure $13 \mathrm{~B}$ shows that neither starvation nor re-stimulation resulted in a changed of FRET efficiencies. These results suggest that the assay was not functional as it did not reflect changes in mTOR phosphorylation.

A

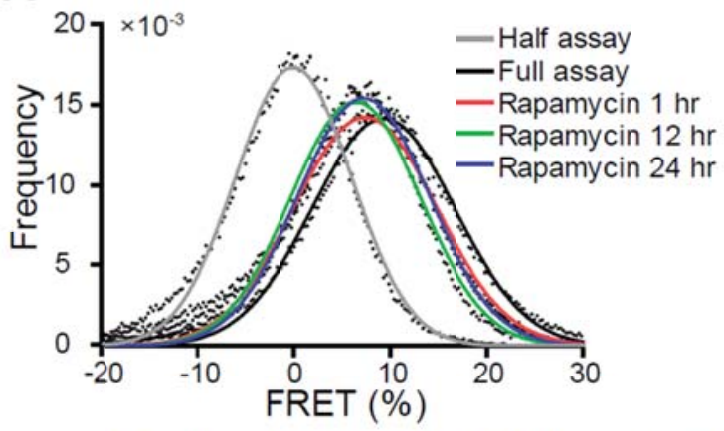

B

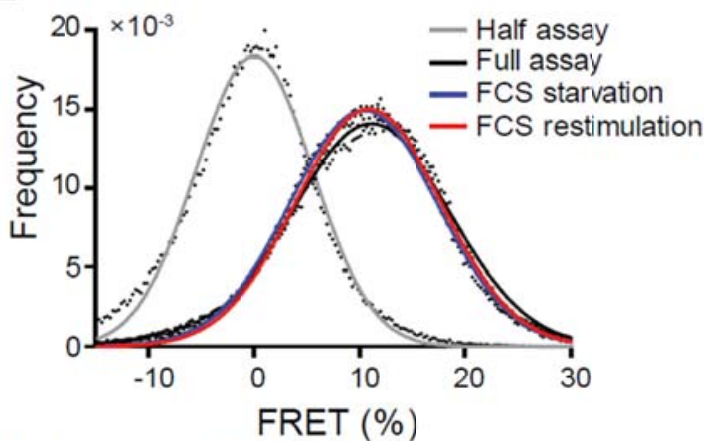

Figure 13: Stimulation and inhibition of mTOR phosphorylation

A: FRET efficiency distributions calculated from fluorescence lifetimes of ATTO 532. Phosphorylation of $\mathbf{S} 2448$ was inhibited by rapamycin. The Half assay denotes the cross-reactivity control, omitting the pS2448 antibody. The FRET efficiency dirstributions were normalised and averaged per condition, $n=8$ cells. B: FRET efficiency distributions of $0.1 \%$ FCS starved cells and restimulated with $10 \%$ FCS cells were normalised and averaged per condition, $\mathrm{n}=5$ cells. 
We suspected that the IHC-specific antibody did not faithfully displays the phosphorylation level of mTOR. We therefore selected a new antibody and tested its phospho-selectivity by a semi-quantitive immunofluorescence experiment. It was tested if the phosphorylation-specific antibody D9C2 termed "XP" could detect changes in the phosphorylation of mTOR. To this end, HEK293 cells were treated with rapamycin for $30 \mathrm{~min}$ and stained with XP-ATTO 532. The relative ATTO 532 fluorescence intensity was measured on a wide-field microscope using constant illumination and acquisition times. The fluorescence intensities were normalized to unity and averaged over 20 fields of view. The results are displayed in Figure $14 \mathrm{~A}$. It shows that rapamycin reduces the intensity of the XP signal, which confirms that the antibody detects reduced phosphorylation levels of mTOR. Consequently, the IHCspecific antibody was replaced by the XP antibody.

Following, it was tested if the XP antibody exhibits FRET with the abcam total mTOR antibody (figure $14 \mathrm{~B}$ ). To this end, the XP was directly compared to the IHC-specific in the Cy3/Cy5-based mTOR assay. The side by side comparison showed that the XP antibody exhibits 8.7 \% FRET (SD: $7.7 \%$ ) compared to $4.9 \%$ FRET (SD: $8.3 \%$ ).

It was next tested if the mTOR assay using the XP antibody would detect changes in mTOR phosphorylation as induced by rapamycin or insulin. Yet again, the assay failed to reflect changes of its biological substrate (Figure $14 \mathrm{C}$ ). 

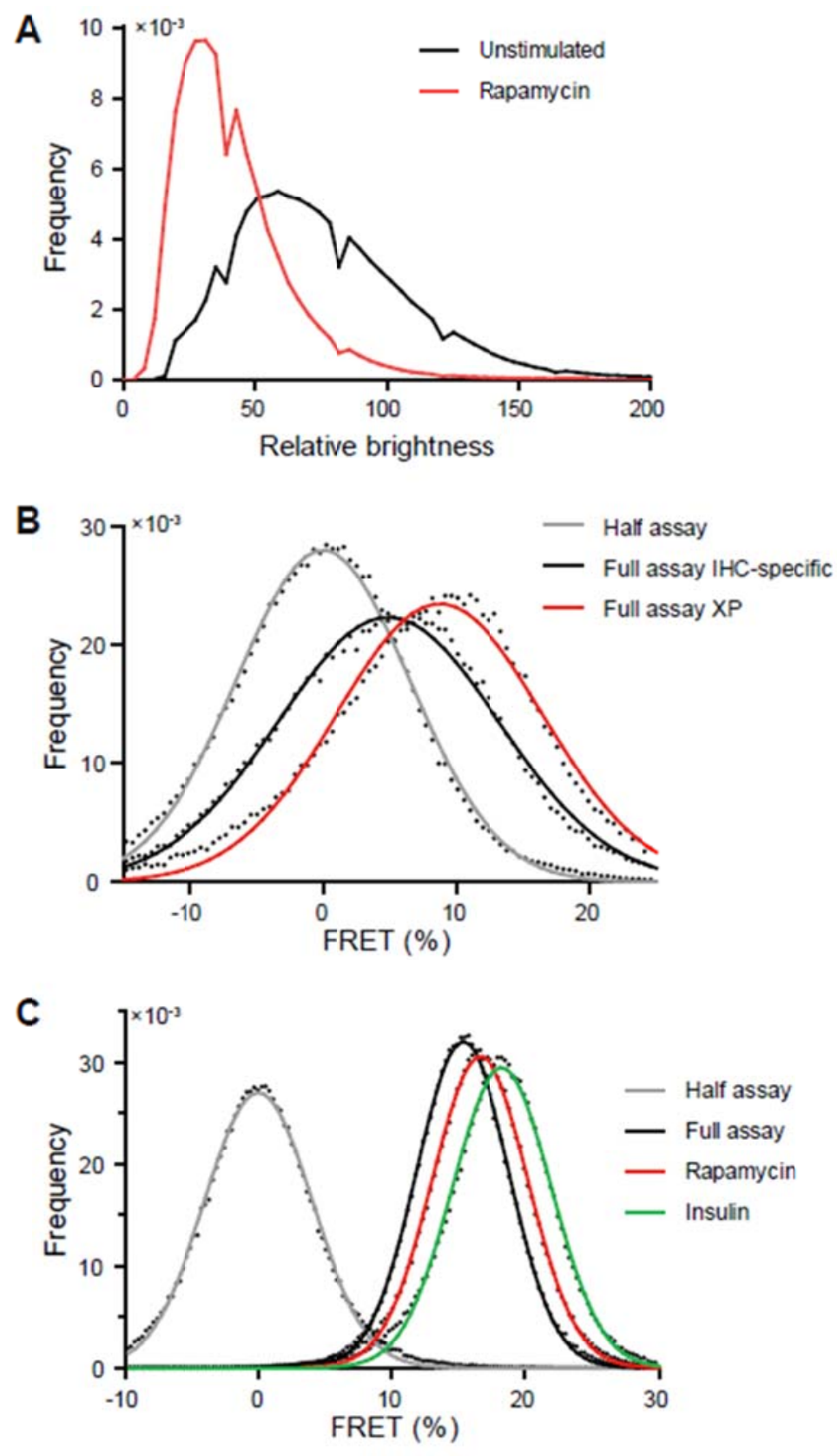

Figure 14: XP antibody replaced for IHC specific

A: REF52 cells were stained for pS2448-ATTO 532 and fluorescence intensity measured on a camera based system with fixed illumination and aquisition times. 20 fields of view were recorded, normalised and averaged per condition. Phosphorylated S2448 was inhibited by rapamycin. B: REF52 cells were stained for mTOR-ATTO 532 and pS2448-ATTO 580Q either with the XP or the IHC-specific antibody. FRET efficiency distributions were calculated from the fluorescence lifetimes of ATTO 532, $n=5$ cells. C: Evaluation of the mTOR phosphorylation assay comprising the XP antibody by modulating S2448 phosphorylation. FRET efficiency distributions calculated from the fluorescence lifetime of untreated, insulin- or rapamycin-treated cells are show, $n=10$ cells. 
After eliminating the phosphorylation-specific antibody as reason for failure, we focused on the secondary antibodies. In each experiment, a cross-reaction control termed "half assay" was made by incubating the decorated first primary antibody with the second secondary antibody (see Figure 7). However, there is an additional mechanism of cross-reactivity that was not considered yet: antigen trapping.

Bivalent $\mathrm{F}(\mathrm{ab})_{2}$ fragments have two antigen-binding domains, with each domain having the capacity to bind an epitope individually. If $F(a b) 2$ fragments bind their epitope with only one of the antigen-binding domains, one remains free. The unbound antigen-binding domain could capture a primary antibody during the incubation with the succeeding primary antibody. The "trapped" primary antibody is succeedingly decorated with acceptor-conjugated $F(a b)_{2}$ fragments, which, in this configuration is very likely to generate high FRET efficiencies due to guaranteed proximity of less than $10 \mathrm{~nm}$ (Figure 15). The resulting overlapping clouds of labelled secondary $\mathrm{F}(\mathrm{ab})_{2}$ fragments would ensure high FRET efficiencies which are independent of epitope recognition by the phosphorylation-specific antibody.

This is exactly what was observed in the previous experiments (Figures 13 and 14). The only available option to circumvent antigen trapping is the use of antibodies with singular binding domains or labelled primary antibodies. The use of labelled primary antibodies is very expensive and the signal to noise ratio is low compared secondary antibodies.

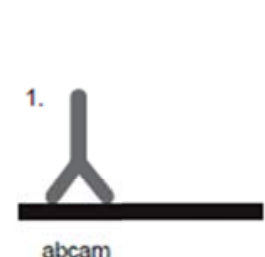

abcam

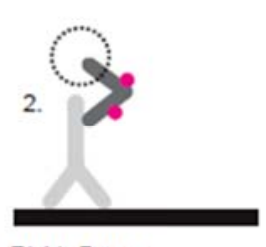

$\mathrm{F}(\mathrm{ab})$,-Donor

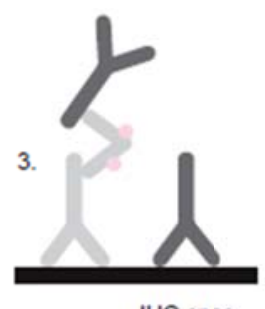

IHC-spec.

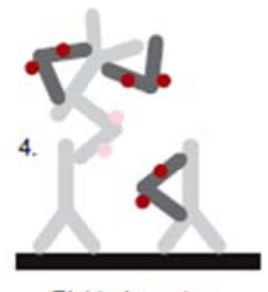

$\mathrm{F}(\mathrm{ab})_{2}$-Acceptor

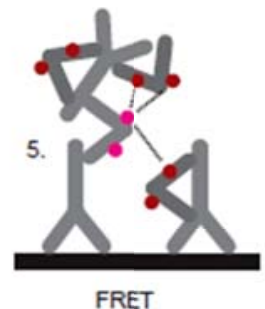

FRET

Figure 15: Schematic for antigen trapping

The use of two antibodies from the same species in sequential staining has the inherent problem of antigen trapping. Free antigen binding domains (dashed circle) of ' $F(a b)_{2}$-Donor' (2) will trap 'IHC-spec.' (3), which will than be decorated with $F(a b)_{2}$-Acceptor (4). This mechanism brings both labels in close proximity which allows for FRET to occurr (5), independently of the epitope recognition by 'IHC-spec.'. 


\subsubsection{Monovalent Fab fragments in antibody-based FRET assay}

The only single-domain antibodies, commercially available at the moment, are camelid single domain antibodies (sdAb, also marketed as nanobodies) and monovalent Fab fragments. Camelids produce antibodies with antigen binding domains consisting only of a variable heavy chain ( $\mathrm{V}_{\mathrm{HH}}$ domain). The $\mathrm{V}_{\mathrm{HH}}$ domain represents a 12-15 $\mathrm{kDa}$ immunogenic fragment with full antigen binding capacity. In comparison, monovalent Fab fragments derived from IgGs are $55 \mathrm{kDa}$ in size. Due to their lower size nanobodies allow higher labelling densities than Fab fragments. However, at the time that these experiments were executed, this technology was new and nanobodies directed against mTOR, pS2448 or primary rabbit antibodies were not available. The custom design of nanobodies involves the immunization of Lamas and subsequent genetic screenings, which costs time and money. In contrast Fab fragments are an established technology, well studied, easily available and cheap.

Hence, monovalent Fab fragments were conjugated to ATTO 532 or ATTO $580 \mathrm{Q}$ and used as secondary antibody in the phosphorylation assay. The labelling ratio was determined as 0.95 or 1.17 labels per Fab fragment. As there was no experience in the use of monovalent Fab fragments, the first step was to determine the optimal antibody dilutions. To this end HEK293 cells were stained with anti-mTOR 7C10 and different dilutions of ATTO 532-conjugated Fab fragments. The relative intensity and lifetime of ATTO 532 was determined by FLIM. At higher antibody concentrations, the fluorescence intensity was higher and the standard deviations of the recorded lifetimes smaller. This may be due to autofluorescence that is contributing more to the overall signal at low antibody concentrations. A good signal-to-noise ratio was obtained at a dilution of 1:200.

In order to determine the cross-reactivity between the Fab fragments, the ability to infiltrate an already existing Fab decoration was tested. To this end, mTOR was stained with ATTO 532-conjugated Fab fragments at 1:200 and was subsequently incubated with differing dilutions of ATTO 580Q-conjugated Fab's. The results show that ATTO 580Q-conjugated Fab's penetrate the preexisting ATTO 532 decoration at every dilution tested. At 1:500 the compromise between labelling and cross-reactivity appeared to be optimal.

In the opposite configuration the competition between ATTO 532-conjugated Fab fragments and an existing ATTO 580Q-Fab decoration was tested. As in the previous 
experiment, the binding of ATTO 532-Fab's is increasing proportionally with its concentration. At concentrations higher than 1:100 the ATTO 532-Fab starts to penetrate the ATTO 580Q-Fab decoration more efficiently. It was therefore decided to use ATTO 532-Fab fragments at a dilution of 1 in 200 or lower (Data not shown).

\subsubsection{Physiological evaluation of the mTOR assay}

After defining antibody dilutions, cross-contamination and staining sequence with the new conjugated Fabs, the physiological responsiveness was to be evaluated. HEK293 cells were stained with thte XP antibody in combination with the 7C10 or the abcam (Figure 16). For comparability, both experiments were performed in parallel. The 7C10 antibody showed the already described granular staining pattern with mTOR beeing localized to intracellular membranes. The mean FRET efficiency of the full assay is $5.0 \%$ (SD: $2.48 \%$ ). The administration of rapamycin for 1 hour reduces the FRET efficiency to $2.7 \%$ (SD: $2.4 \%$ ). The granular structures that are localised closely behind the leading edge of the cell seem to react stronger to rapamycin than the more central ones. This is in agreement with the literature (Sancak et al. 2010; Zhou et al. 2013)

When the mTOR assay is performed with the abcam antibody the staining pattern shows a strong nuclear localisation (Figure 6). The localisation of mTOR in the cytoplasm and the plasma membrane is consistent with the 7C10 staining. The unstimulated cells show FRET efficiencies of $8.8 \%$ (SD: $2.3 \%$ ). The administration of rapamycin reduced the FRET efficiency to $7.7 \%$ (SD: $2.1 \%$ ). The response to rapamycin is strongly inhomogeneous with reduced FRET efficiencies in the cytoplasm while the nuclear signal remained unchanged. This suggests that the nuclear localisation observed with the abcam antibody may originate from unspecific binding to a different target that shows nuclear localisation. The $7 \mathrm{C} 10$ was therefore considered to represent the more correct staining pattern. 

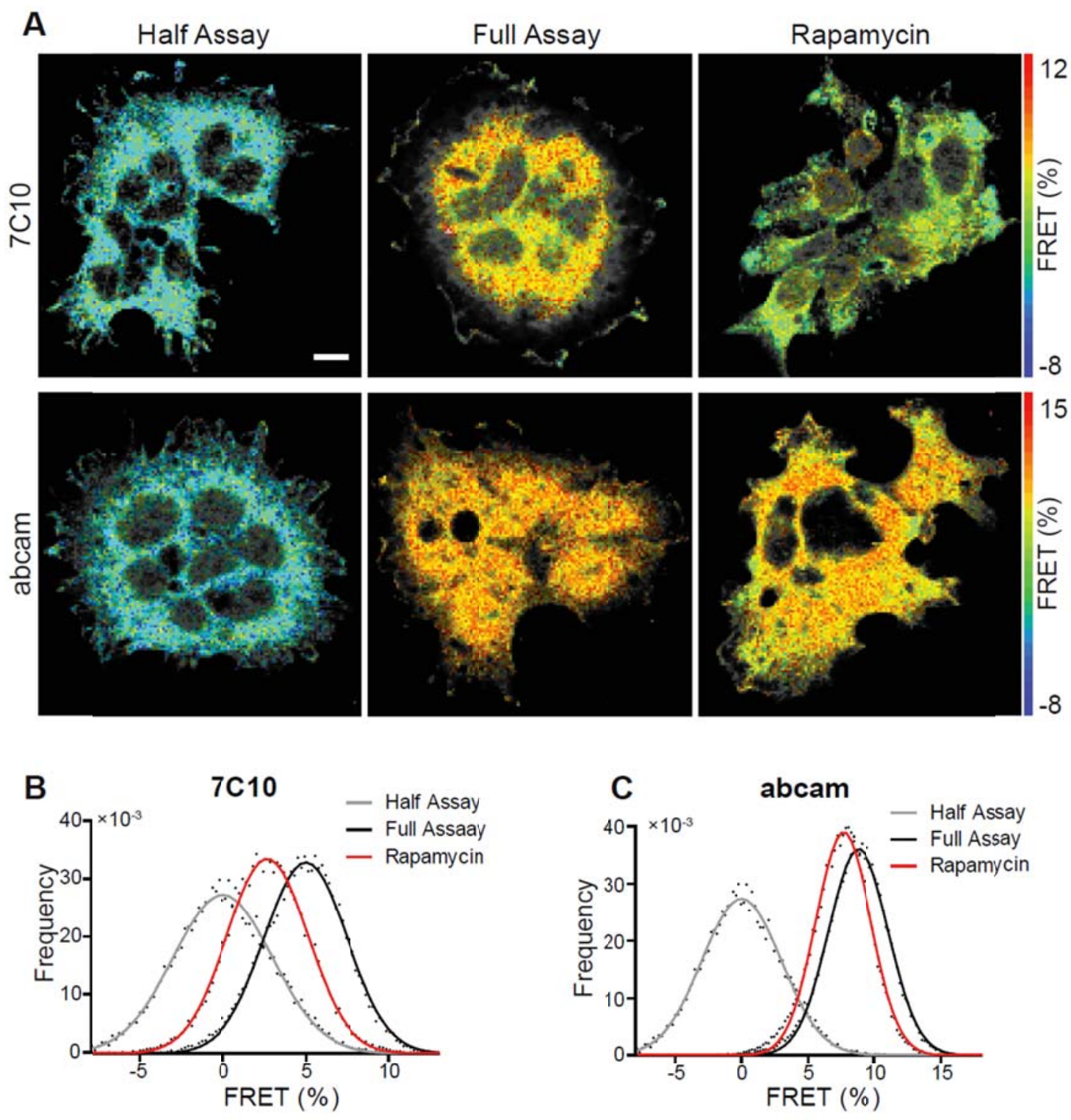

Figure 16: Comparison between the 7C10 and abcam antibody

A: Representative fluorescence lifetime images overlayed with the corresponding intensity images (false colour) of HEK293 cells stained with either abcam or $7 \mathrm{C} 10$ antibody against mTOR-labelled ATTO 532. pS2448 was labelled with ATTO 580Q. Scale bar $10 \mu \mathrm{m}$. B: FRET efficiency distributions calculated from fluorescence lifetime distributions per condition are shown, $n=5$ fields of view. C: FRET efficiency distributions calculated from fluorescence lifetime distributions per condition are shown, $n=5$ fields of view. 
From the data in Figure 14 it was known that the XP does react to rapamycin in a semi-quantitative assay. On the other hand, it was also known that the IHC-specific failed yet to respond to physiological stimulations. In order to verify this, both antibodies were compared side by side in the mTOR assay (Figure 17). As in the previous Figure the XP antibody showed approximately $5 \%$ (SD: $1.6 \%$ FRET efficiency. When mTORC1 was inactivated with rapamycin, the FRET efficiency was reduced to $0.5 \%$ (SD: $2.4 \%$ ). In the previous experiment the reduction was smaller here maybe because a lower concentration (1:500) of ATTO 532-Fab fragments was used.

In comparison, the IHC-specific antibody gave a higher mean FRET efficiency of 8.7 \% (SD: $1.3 \%$ ). But rapamycin reduced the FRET efficiencies only to $6.0 \%$ (SD: 2.7 \%). The residual FRET was consistently located to the perinuclear region as well as to protrusions and ruffles of the plasma membrane while the cytoplasmic FRET signal was consistently reduced. The XP antibody seemed therefor to be more specific than the 'IHC-specific' in detecting changes of mTOR phosphorylation and was therefore used in all following experiments.

The mTOR phosphorylation assay, as it was established here uses a staining procedure starting with the pS2448 (XP) which is decorated with an ATTO 580Qconjugated monovalent Fab fragment at a dilution of 1:200. Total mTOR is detected by the 7C10 antibody and decorated with an ATTO 532-conjugated monovalent Fab fragment at a dilution of 1:500. The previous experiments demonstrated that antibody-based FRET assays can be established even if both primary antibodies are raised in the same species. By using monovalent Fab fragments the cross-reactivity could be controled. Moreover, the here presented results demonstrate how crucial high antibody specificity is and how FRET can be used to identify unspecific binding. 
A
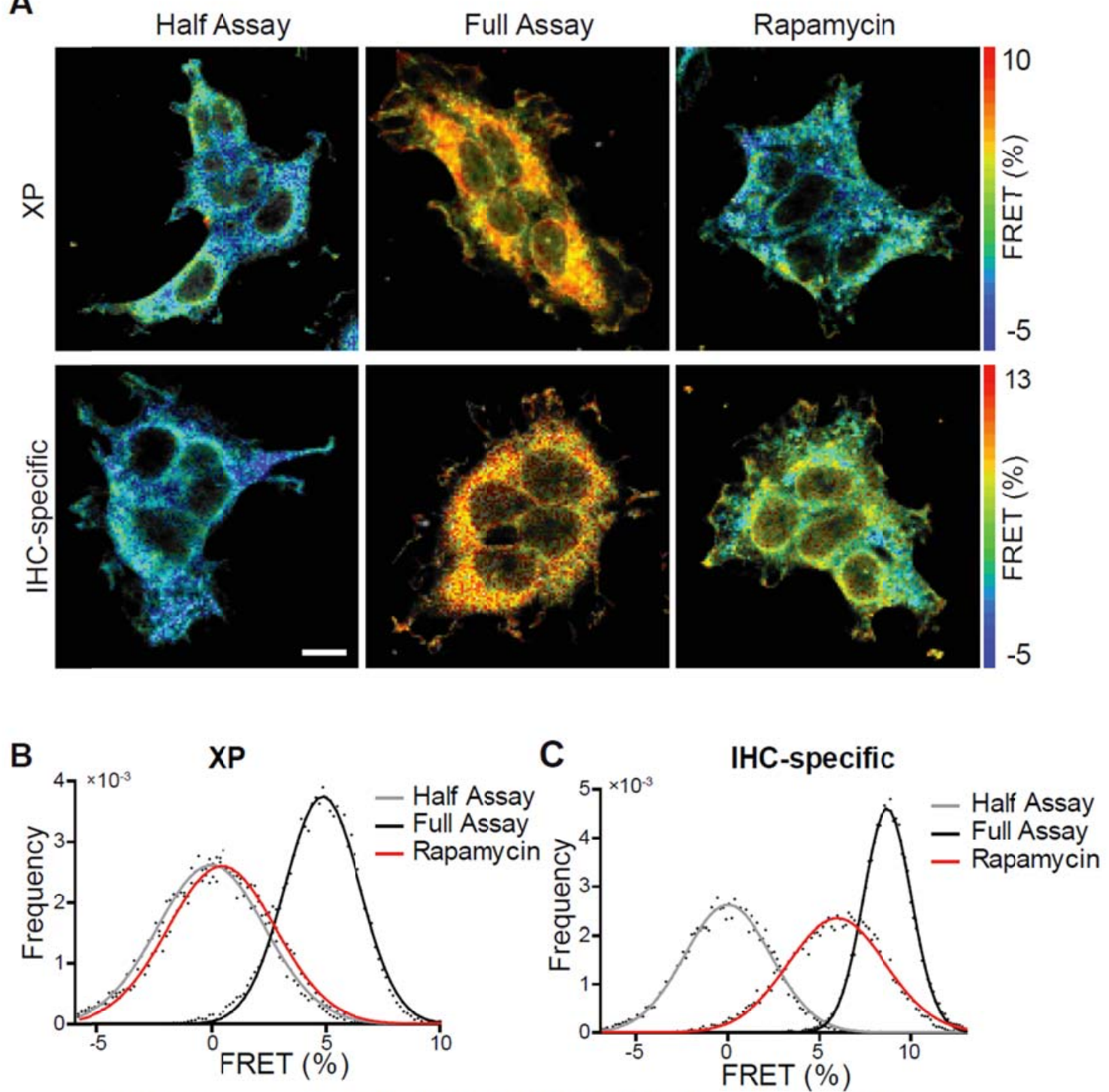

Figure 17: Direct comparison between the XP and IHC-specific antibody

A: Representative FRET efficiency images overlayed with the corresponding intensity image (false colour) of HEK293 cells stained with either XP or IHC-specific antibody against pS2448labelled ATTO 580Q. Total mTOR was labelled with ATTO 532. Scale bar $10 \mu \mathrm{m}$. B: FRET efficiency distributions calculated from fluorescence lifetime distributions per condition are shown, $n=5$ fields of view. C: FRET efficiency distributions calculated from fluorescence lifetime distributions per condition are shown, $n=5$ fields of view. 


\subsection{7 mTORC1 signalling in MeCP2-KO neurons}

The mTOR assay was designed to allow for analysis of mTORC1 activity on single cell level. This was supposed to be applied to MeCP2-KO neurons in order to identify potential sub-populations of brain cells that show reduced mTORC1 activity. Neuronal cell cultures of the hippocampus of MeCP2-KO mice and WT littermates were prepared and fixed at DIV7. The mTORC1 assay was applied according to the procedure described in previous experiments.

The staining shows that mTOR is localised in the cytoplasm with a very pronounced staining in the soma, dendrites and axons. The granular localisation is less pronounced in neurons than in HEK293 cells. As observed in HEK293 cells, the nucleus does not contain any mTOR signal. The phosphorylation assay shows that mTORC1 activity varies strongly between cells. Neurons appear to have higher phosphorylation than smaller cells that appear to be glia. Hippocampal neurons from MeCP2-KO mice show the same mTOR localization as WT but the overall phosphorylation level is significantly reduced ( $2.5 \% \mathrm{SD}$ ). Cells that, on the basis of their morphology are judged to be neurons, show very low FRET efficiencies and are smaller, show less dendritic arborisation and thinner axons than in the WT preparation. Cells with glia appearance have higher FRET efficiencies than most neurons in preparations from MeCP2-KO mice. The overall fitness and viability of MeCP2-KO neurons was judged lower than of WT neurons (Figure 18). A higher number of dead cells and a lower neuron to glia ratio were observed, which taken together may suggest a neuron-specific reduction of mTOR phosphorylation leading to decreased cell viability. These findings are in line with a study from Ricciardi et al. (2011) that showed reduced S2448 phosphorylation and reduced S6K1 activity in brain lysates of MeCP2-KO mice.

The lifetime distributions under half assay conditions show a difference between WT ( $2.5 \mathrm{~ns})$ and MeCP2-KO (2.35 ns) even though the assay conditions are the exact same. Cross-reactivity is, as long as the number of epitopes is the same, a mere function of the concentrations of the two secondary antibodies. Here the antibody concentrations were the same. Hence the lifetime difference under 'half assay' conditions may be due to changes in the number of epitopes. The reduced lifetime in MeCP2 may reflect a lower mTOR expression as a direct effect of MeCP2 inactivation. This would suggest that a reduced expression as well as 
phosphorylation of mTOR may be responsible for the neuronal defects caused by MeCP2 inactivation.

A WT Half Assay
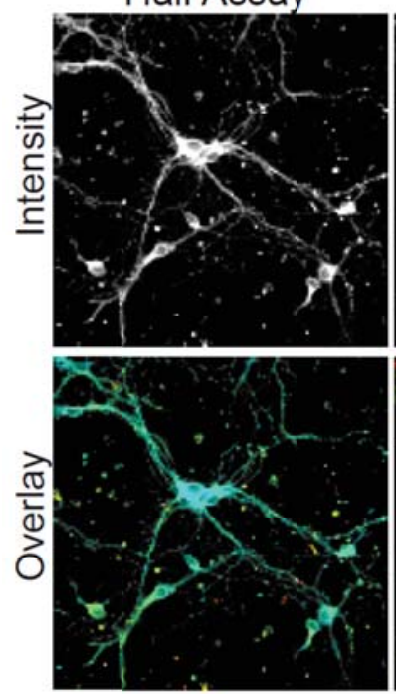

WT Full Assay
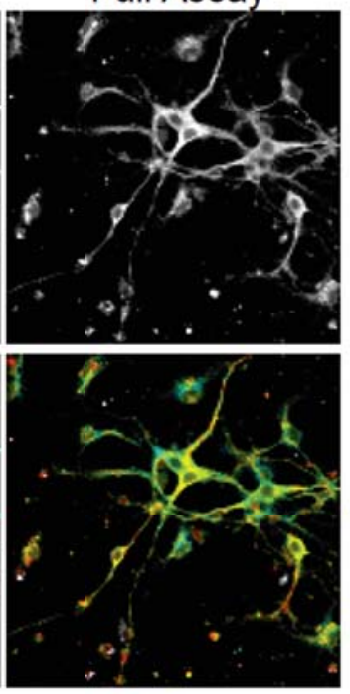

MeCP2-KO Half Assay
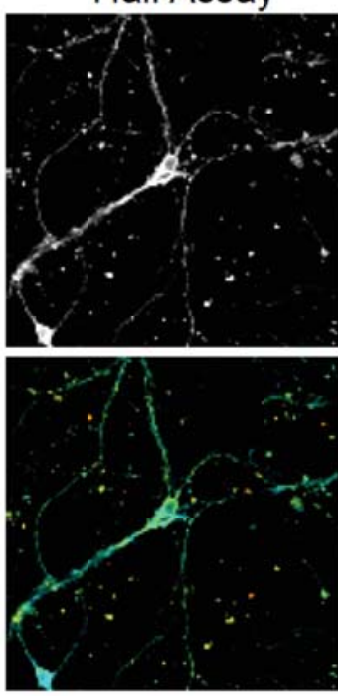

MeCP2-KO
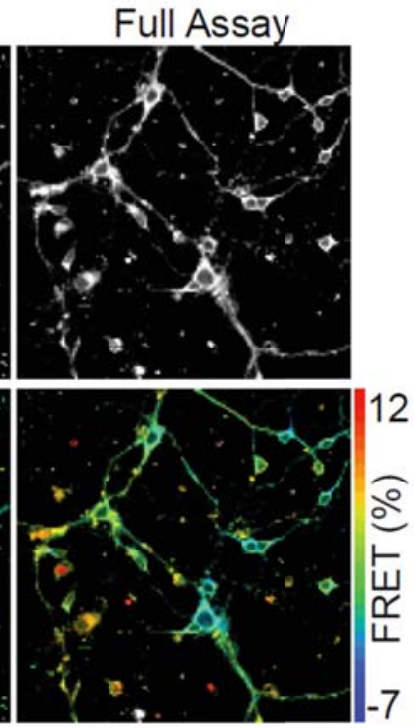

\section{B}
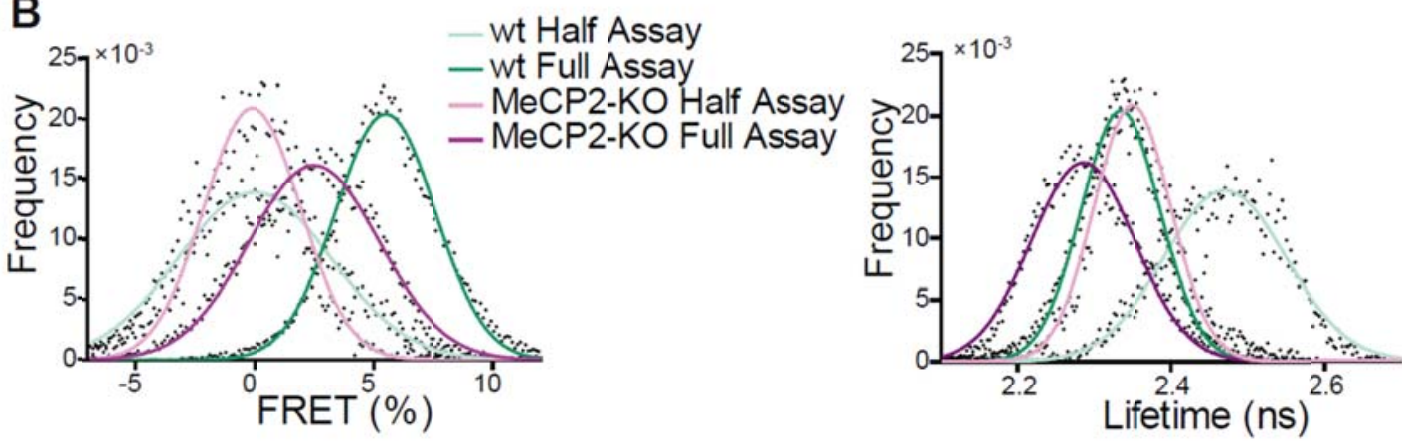

Figure 18: Reduced mTOR phosphorylation in MeCP2-KO neurons

A: Representative intensity (top row) (grayscale) and FRET efficiency images overlayed with the corresponding intensity images (bottom row) (false colour) of primary hippocampal neurons derived from WT or MeCP2-KO mice were fixed DIV 12 and stained with mTORATTO 532 and pS2448-ATTO 580Q. Scale bar $10 \mu \mathrm{m}$. B: FRET efficiency distributions or fluorescence lifetime distributions per condition are shown, $n=8$ fields of view. 


\subsection{Development of an mTORC2 activity assay}

Much effort has been put into elucidating the signalling pathways linked to mTORC1. Many upstream activators and downstream effectors have been identified and characterized. They make up a complex signalling network that mTORC1 is embedded in.

In contrast to mTORC1, very little is known about mTORC2. It is activated by insulin, growth factors and nutrients and regulates the activity of Akt and PKC $\alpha$.

The activation mechanism of Akt includes its recruitment to the plasma membrane, where it is phosphorylated by PDK-1 at T309. This renders Akt active, but it needs phosphorylation at $\mathrm{S} 473$, executed by mTORC2, to fully activate Akt. Akt activates mTORC1 by inhibiting phosphorylation of TSC2, Pras 40 and GSK-3ß. The phosphorylation of Akt at $\mathrm{S} 473$ seems to be exclusive to mTORC2. Consequently this phosphorylation can conveniently be used as a measure for mTORC2 activity. Analysing this phosphorylation event tells us two things, first: the activity state of Akt itself, and second: that of the executing kinase complex, mTORC2.

As for mTORC1, the most direct way to optically analyse endogenous mTORC2 activity without introducing genetic sensors was by phosphorylation-specific antibodies. This time the mTORC2 substrate, Akt was chosen as epitope because there weren't any good antibodies against mTORC2 subunits available that would show FRET (Data not shown). The best antibodies against Akt according to the rules from section 4.1.1 were raised in rabbit. The experience made with the mTORC1 assay, could therefore be use in the mTORC2 assay (Figure 19).

The phosphorylation-specific antibody shows a staining that is most prominent at the plasma membrane. The cytoplasm and the nucleus show lower intensities. The total Akt signal shows the same staining pattern with a slightly less pronounced membrane localisation in unstimulated cells. Both localisation patterns are in line with the activation process of Akt.

The phosphorylated S473 was stained with ATTO 532-conjugated Fab fragments and total Akt was stained with ATTO 580Q-conjugated Fab fragments. The epitopes of the total-Akt antibody and pS473-Akt antibody are located closely together in the amino acid sequence of Akt. Should the antibodies exhibit steric hindrance in the recognition of the epitopes, it is beneficial to first include the acceptor labelled 
antibody. This way, at least all pAkt epitopes are labelled. This is essential for allowing maximal FRET efficiencies.

The mean FRET efficiencies in unstimulated cells is $15.3 \%$ (SD: $5.1 \%$ ). Upon stimulation with $6.6 \mathrm{nM}$ insulin, high signals from pS473-Akt are detected at the plasma membrane while the administration of LY294002, an inhibitor of PI3K leads to a complete loss of the pS473-Akt signalall over the cell. This confirms the high specificity of the pS473 antibody. The FRET efficiencies decrease by stimulation with insulin (12.9\%, SD: $4.6 \%$ ) and increase considerably by inhibition with LY294002 (23.9 \%; SD: $5.0 \%$ ). This inversion of the FRET signal is caused by the fact that the phosphorylation-specific antibody was labelled with the donor fluorophore (see section 4.1.2). The inhibition of Akt phosphorylation abrogates the binding of the phosphorylation-specific antibody. Consequently, the donor signal is nearly lost, which results in very low donor-acceptor ratios, which favour high FRET efficiencies. In contrast, when phosphorylation of Akt is stimulated by insulin, the FRET efficiency is decreased although Akt is visibly activated, as judged by the massive membrane recruitment. This recruitment results in an increase in the donor-acceptor ratio which is unfavourable for FRET as reflected by reduced FRET efficiencies. 
A
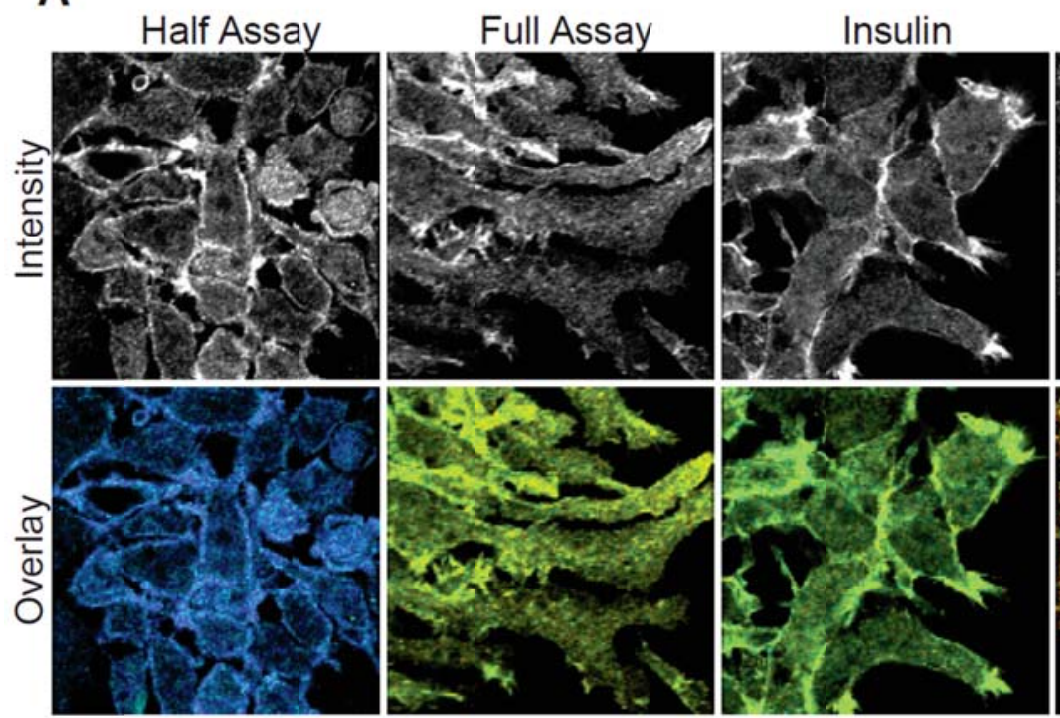

LY294002

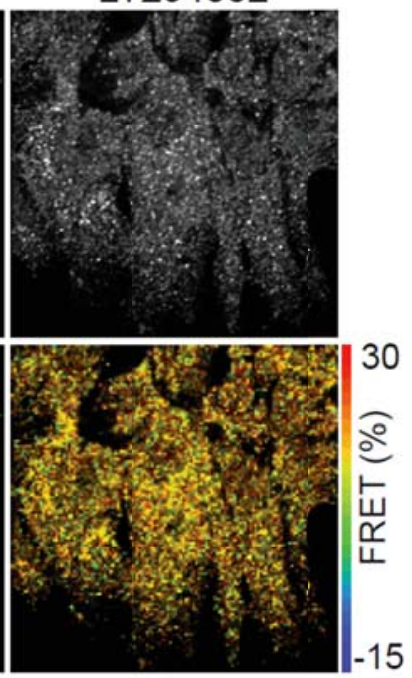

B

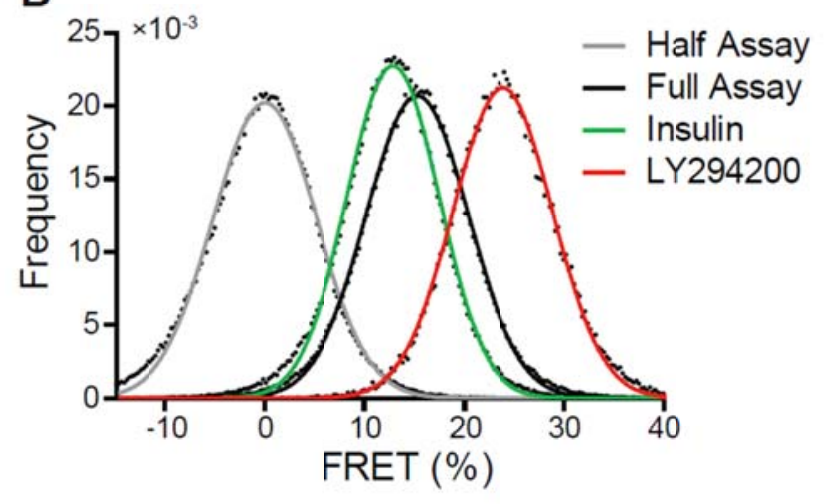

Figure 19: Akt phosphorylation assay with inverted response

A: Representative intensity (top row) (grayscale) and FRET efficiency images overlayed with the corresponding intensity image (bottom row) (false colour) of HEK293 cells were treated insulin or LY294002 and stained for pS473-Akt labelled with ATTO 532 and total Akt labelled ATTO 580Q. Scale bar $10 \mu \mathrm{m}$. B: FRET efficiency distributions calculated from fluorescence lifetime distributions per condition are shown, $n=5$. 
The inverted response was considered not favourable for an Akt activity assay. Therefore, the donor and acceptor labels were switched. Total Akt was now labelled with the donor and pS473-Akt was labelled with the acceptor (Figure 20). In the switched configuration unstimulated cells show a mean FRET efficiency of $4.3 \%$ (SD: $3.9 \%$ ) with high FRET efficiencies of up to $12 \%$ located in membrane ruffles. Akt signal coming from the nucleus showed low FRET efficiencies. The stimulation of Akt with insulin resulted in a strong recruitment of Akt to the plasma membrane where it shows the highest FRET efficiencies of up to $16 \%$. The mean FRET efficiency is increased by $46 \%$ to $6.3 \%$ (SD: $3.8 \%$ ). In contrast, the inactivation of PI3K resulted in a complete loss of membrane-bound Akt and a global loss of FRET $(E=-0.3 . \%$; SD: $4.3 \%)$. These results are in agreement with the already described activation mechanism of Akt and show that the inverted design permits sensitive monitoring of Akt activity. 

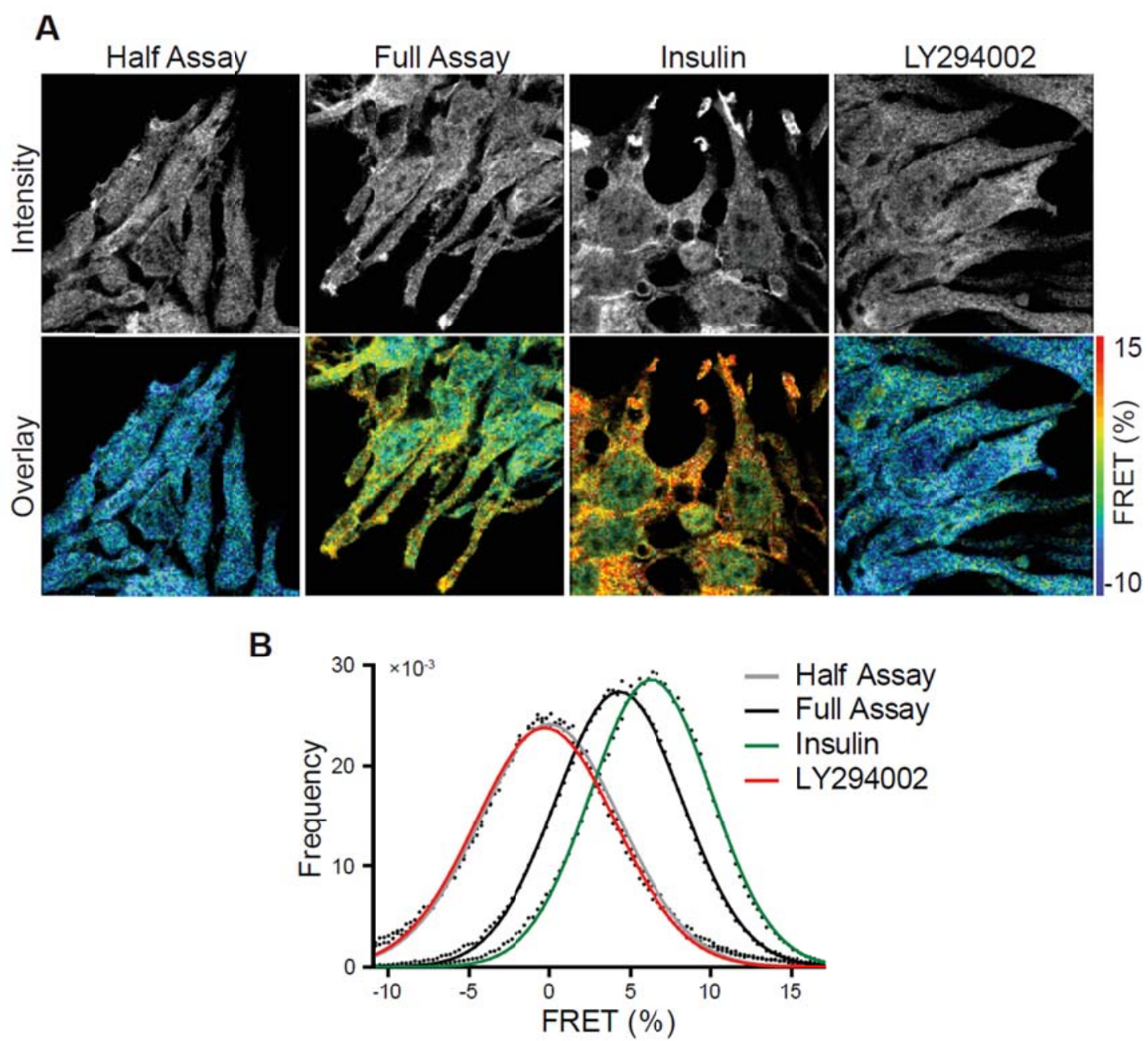

Figure 20: Akt phosphorylation assay

A: Representative intensity (top row) (grayscale) and FRET efficiency images overlayed with the corresponding intensity images (bottom row) (false colour) of HEK293 cells, treated with insulin or LY294002 were stained for PS473-Akt labelled with ATTO 580Q and total Akt labelled ATTO 532. Scale bar $10 \mu \mathrm{m}$. B: FRET efficiency distributions calculated from fluorescence lifetime distributions per condition are shown, $n=5$ fields of view. 
mTORC2 was first reported to be a rapamycin-insensitive second mTOR complex (Sarbassov et al., 2004). As a potential cancer therapeutic rapamycin could reduce mTORC1 signalling without interfering with mTORC2.

Later a cell-type dependent rapamycin-sensitivity to chronic rapamycin administration was described by the same authors (Sarbassov et al., 2009). A potential interfering with both mTOR complexes was considered to be disadvantageous for a potential cancer therapy as it could lead to uncontrolled cell death. One of the described cell types with a reported partial rapamycin-sensitivity of mTORC2 was HEK293. It was therefore tested if rapamycin-sensitivity of $\mathrm{mTORC} 2$ could be reproduced by longterm administration. HEK293 cells were treated with 100 nM rapamycin for 1, 24 or 48 hours and Akt phosphorylation was assayed (Figure 21). The results show that rapamycin does not interfere with the phosphorylation of Akt by mTORC2 as indicated by the FRET efficiency images and distributions. Rapamycin even seems to increase the membrane recruitment of Akt, leading to a significant increase of Akt phosphorylation after 48 hours from $9.7 \%$ (SD: $2.6 \%$ ) to $11.2 \%$ (SD: $2.3 \%$ ) FRET.

The increase in Akt phosphorylation can be explained by an mTORC1-dependent negative feedback mechanism: active mTORC1 effector protein S6K can initiate the sequestration of insulin receptor substrate, an upstream co-activator of the PI3K-AktmTOR pathway. With rapamycin inhibiting this mTORC1-dependent negative feedback mechanism, IRS is enriched at the plasma membrane which fuels the activation of Akt. 
A
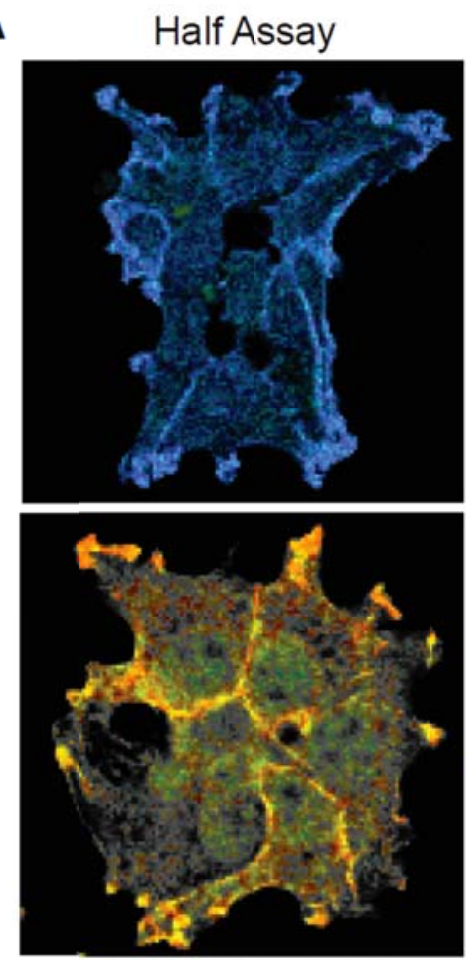

Rapamycin $1 \mathrm{hr}$
Full Assay
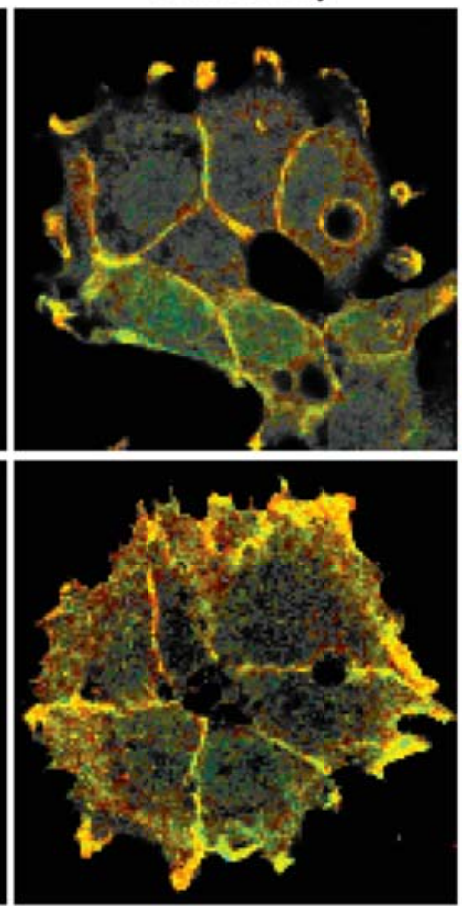

Rapamycin $24 \mathrm{hr}$
LY294002 $1 \mathrm{hr}$

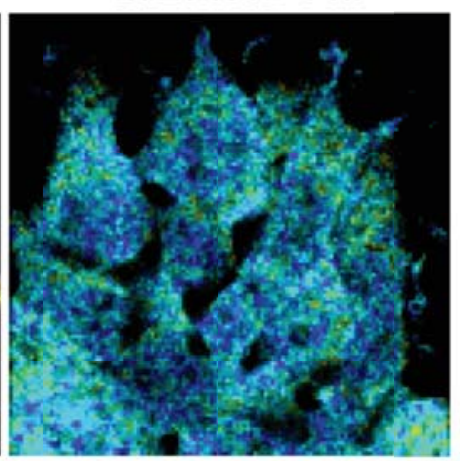

(1)

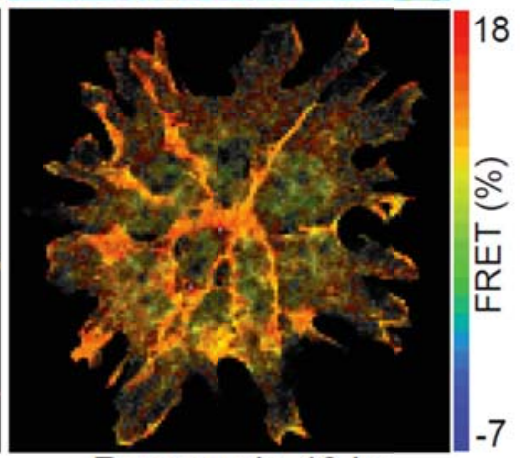

Rapamycin $48 \mathrm{hr}$

— Half Assay

- Full Assay

— LY294002 $1 \mathrm{hr}$

- Rapamycin $1 \mathrm{hr}$

- Rapamycin $24 \mathrm{hr}$

- Rapamycin 48 hr

Figure 21: Chronic rapamycin treatment increases Akt phosphorylation at S473

A: Representative FRET efficiency images overlayed with the corresponding intensity images of HEK293 cells treated with LY294002 or rapamycin were stained for pS473-Akt labelled with ATTO 580Q and total Akt labelled ATTO 532. Scale bar $10 \mu \mathrm{m}$. B: FRET efficiency distributions per condition are shown, $\mathrm{n}=5$. 


\subsubsection{Application of the Akt phosphorylation assay to MeCP2-KO neurons.}

The reduced activation of mTORC1 in neurons derived from MeCP2-KO mice indicates a compromised activation of the PI3K-Akt-mTOR pathway. In neurons, BDNF activates this pathway by binding to the TrkB receptor. It has been reported that BDNF translation, transport and secretion is reduced in MeCP2-KO mice ( $\mathrm{Li}$ and Pozzo-Miller; 2014). To test if mTORC2 activity is reduced in neuronal cultures derived from MeCP2-KO mice, Akt phosphorylation was assayed as established before. In mouse neurons, the Akt staining is located to the plasma membrane and the cytoplasm, with highest intensities located in the soma and major axonal and dendritic protrusions. Relative Akt phosphorylation is homogeneous throughout different cell types and cellular regions (5.1 \% FRET; SD: 2.2 \%). In MeCP2-KO neurons, the mean FRET efficiency is reduced to $2.8 \%$ (SD: $3.0 \%$ ). Like in the mTOR assay, shorter lifetimes were observed under half assay conditions (WT 2.5 ns; MeCP2-KO 2.3 ns) They indicate a reduced level of Akt expression in MeCP2-KO neurons compared to WT. This result is similar to the observation that mTOR expression is reduced in MeCP2-KO neurons. An intriguing possibility would be that a negative feedback mechanism exists by which reduced expression of BDNF results in reduced activation the PI3K-Akt-mTOR signalling pathway which in turn reduces the total protein translation (Figure 25). 
A

WT Half Assay
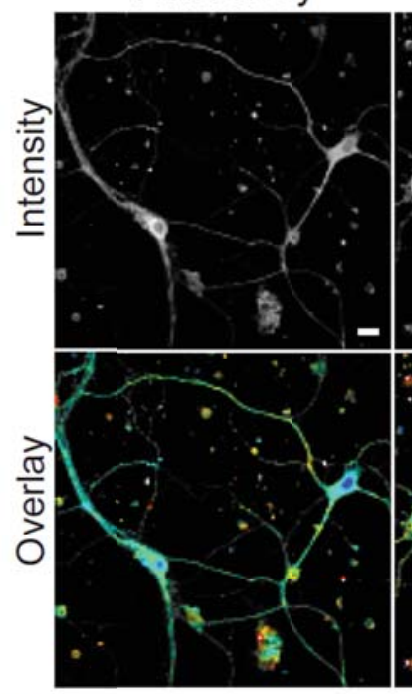

B

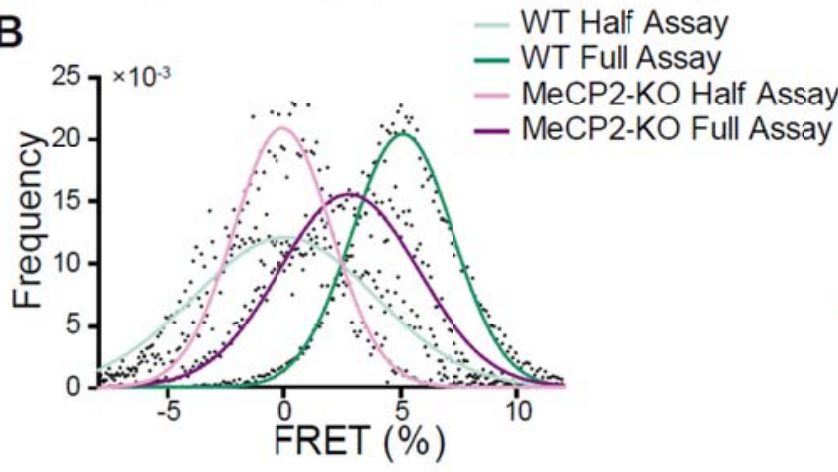

WT Full Assay
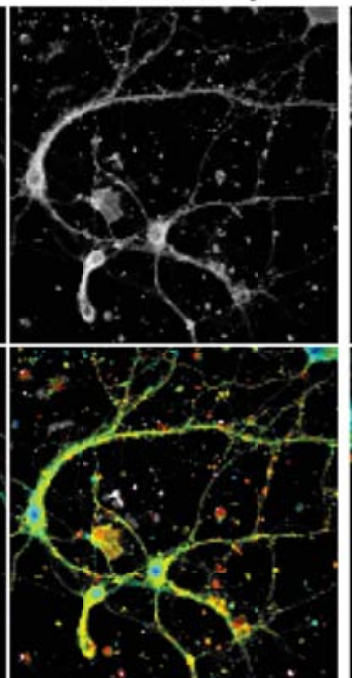
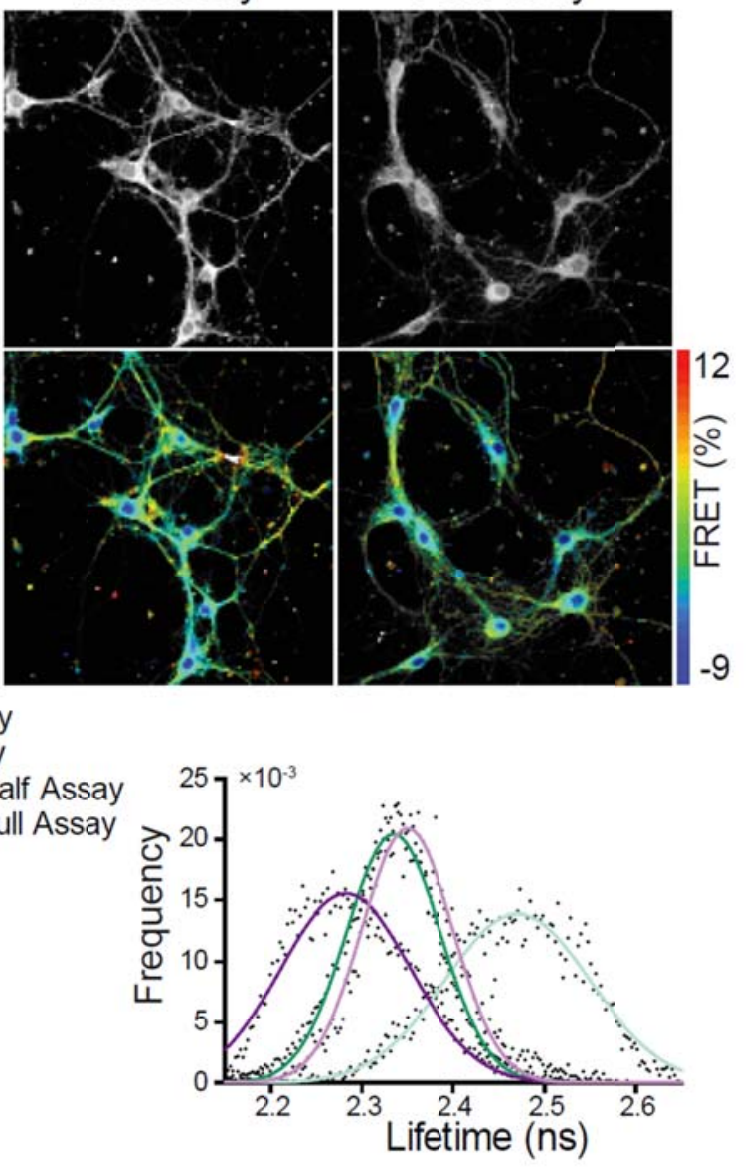

Figure 22: Reduced Akt phosphorylation in MeCP2-KO neurons

A: Representative intensity (top row) (grayscale) and FRET efficiency images overlayed with the corresponding intensity images (bottom row) (false colour) of primary hippocampal neurons derived from WT or MeCP2-KO mice were fixed DIV 12 and stained for Akt-ATTO 532 and pS473-ATTO 580Q. Scale bar $20 \mu \mathrm{m}$. B: FRET efficiency distributions or fluorescence lifetime distributions per condition are shown, $n=8$ fields of view. 


\subsection{A FRET-based mTOR-gephyrin interaction assay}

In 1999, Sabatini and colleagues reported a direct interaction of mTOR and gephyrin with functional implications for mTORC1 signalling. Mutants of mTOR, that were unable to bind gephyrin also failed to activate their downstream targets S6K1 and 4E-BP1. These findings placed mTOR dependent mRNA translation into the context of inhibitory synapse formation, maturation, and plasticity. One major obstacle to define the role of mTOR and gephyrin interaction at inhibitory postsynaptic sites is that both molecules are ubiquitously expressed throughout all cell types. It was therefore indicated to create an optical assay that reports on the interaction of mTOR and gephyrin with spatial resolution. For this purpose, EGFP- and mCherry- fusion constructs of mTOR and gephyrin were co-expressed and FRET was measured by TSCPC-FLIM.

First, it was tested whether EGFP fused to mTOR at the C- or N-terminus results in FRET with mCherry-gephyrin. To this end, both proteins were co-expressed in HEK293 cells. Neither the C- nor the N-terminal fusion construct of mTOR showed FRET with mCherry (data not shown). This finding indicated that the gephyrin binding site within mTOR is too far away from either ends of mTOR for FRET to occur. This is in line with the reported gephyrin binding site being located to the distal portion of the HEAT domain with isoleucine 1034 being crucial for gephyrin binding (Sabatini et al., 1999).

It was therefore tested if an antibody labelling would increase the chance of FRET. Antibody labelling can be used to mitigate the extreme distance dependence of FRET. As already described, a full IgG has a diameter of around $10 \mathrm{~nm}$ and $F(a b)_{2}$ fragment is $5 \mathrm{~nm}$ in diameter. The FRET range can be extended by a theoretical maximum of $15 \mathrm{~nm}$. Additionally, an antibody labelling with polyclonal secondary antibodies creates an unstructured cloud of fluorophores which reduces the orientation dependence of FRET. The 7C10 antibody was used to label the distal portion of mTOR. It was labelled with ATTO $580 \mathrm{Q}$-conjugated $\mathrm{F}(\mathrm{ab})_{2}$ fragments. FRET was measured by determining the lifetime of mCherry-gephyrin. EGFP-mTOR and mCherry-gephyrin were co-expressed in a 1:1 molar ratio and the lifetime of mCherry was measured with or without the mTOR-ATTO $580 \mathrm{Q}$ antibody labelling. mCherry-gephyrin exhibits both a cytoplasmic and a sub-membranous expression. It was never detected in the nucleus. At higher expression levels gephyrin forms large 
amorphous intracellular aggregates that are of globular or amorphous shape. Since large aggregates like these are not reported to exist in neurons, they are considered to be overexpression artefacts and were excluded from the measurements. The abundance of gephyrin aggregates was found to be directly dependent on the amount of cDNA transfected as well as the duration of expression. These parameters were optimized for the reduction of gephyrin aggregation in our experiments. For the measurement of the mTOR-gephyrin interaction, only cells without aggregates were chosen. The intensity and the corresponding FRET efficiency images of gephyrin are shown in Figure 23. Without antibody staining, no FRET could be detected. Cells stained with mTOR-ATTO 580Q display a mean FRET efficiency of 13.5 \% (SD: 2.6 $\%)$. The interaction appears to be homogeneous throughout the cytoplasm. There are regions with granular staining that are likely to represent mTOR-positive intracellular membranes that are also observed in staining with the $7 \mathrm{C} 10$ antibody.

The ability to measure the interaction between gephyrin and mTOR raised the question to which mTOR complex gephyrin binds to. Sabatini and colleagues also addressed this question but could not show a reduced interaction when mTORC1 was disrupted with rapamycin. Instead, they used indirect evidence by showing a reduced activity of S6K1 and 4E-BP1 in cells that expressed mTOR mutants that did not bind to gephyrin.

It was therefore tested if the pharmacological inhibition of mTORC1 and mTORC2 has an impact on association with gephyrin. To this end, rapamycin, a specific mTORC1 inhibitor, or LY294002, an inhibitor of mTORC1 and 2, was applied to cells expressing EGFP-mTOR and mCherry-gephyrin. Figure 23 shows that rapamycin efficiently reduced the interaction between mTOR and gephyrin from $14.3 \%$ FRET to $9.6 \%$ (SD: $2.4 \%$ ) FRET. Administration of LY294002 resulted in the same reduction (8.9\% SD: $2.5 \%)$. This result indicates that gephyrin interacts exclusively with mTORC1 and not with mTORC2. 


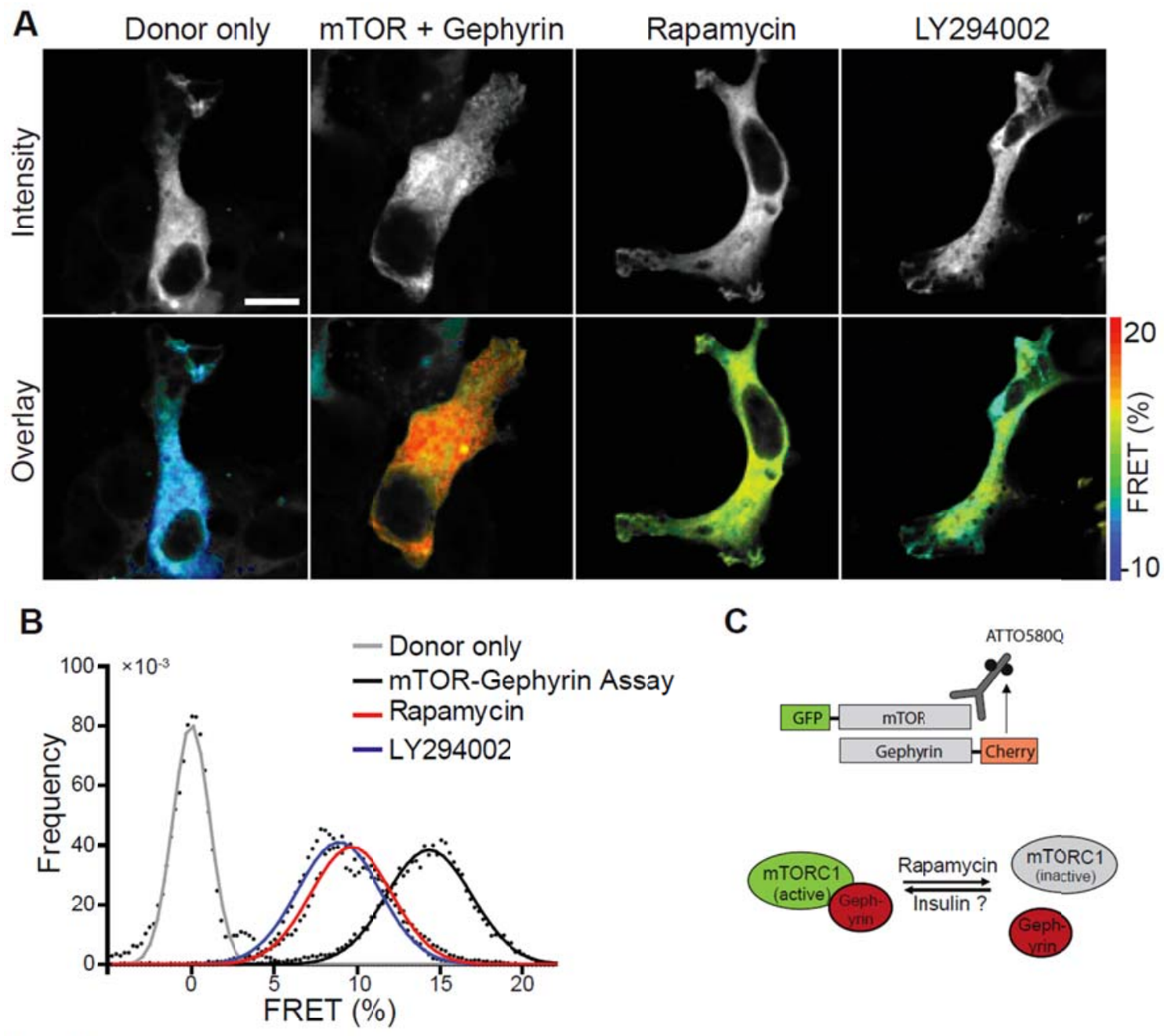

Figure 23: Gephyrin exclusively interacts with mTORC1

A: Representative intensity (top row) (grayscale) and FRET efficiency images overlayed with the corresponding intensity images (bottom row) (false colour) of EGFP-mTOR and mCherrygephyrin, coexpressed in HEK293 cells and stained for the C-terminus of mTOR with the 7C10 mTOR antibody labelled with ATTO 580Q. Cells were treated with rapamycin or LY294002. Scale bar $10 \mu \mathrm{m}$. B: FRET efficiency distributions per condition are shown, $n=5$. C: Schematic description of the assay principle and the mechanism of the mTOR-gephyrin interaction. 
Gephyrin has been reported to be highly phosphorylated with over 20 phosphorylatable serine residues located within its C-domain. In recent reports, some phosphorylation sites could be connected to kinases: i.e. ERK1/2 at S268 and CDK5, and GSK-3 $\beta$ at S270. Moreover, Pin1 has shown to interact with phosphorylated S200.

Alanine exchange mutations of S200, S268 and S270 were introduced into mCherrygephyrin and analysed for their interaction with EGFP-mTOR.

The ectopic expression of gephyrin mutants did not change the localization of gephyrin or the morphology of the cell. The analysis of the interaction with mTOR revealed that the S200A as well as the S268A showed comparable FRET efficiencies with WT gephyrin. The gephyrin mutant S270A, however, showed reduced interaction with mTOR. This result indicates that S270 is involved in the interaction between mTOR and gephyrin (Figure 24). It also shows that this phosphorylation site is likely to be phosphorylated in a state of cellular growth as the exchange mutant would not have had any effect. The third conclusion that can be drawn from this and the previous experiment is that the interaction between mTOR and gephyrin is multistep process with prerequisites on both sides, which speaks for a highly regulated process. 


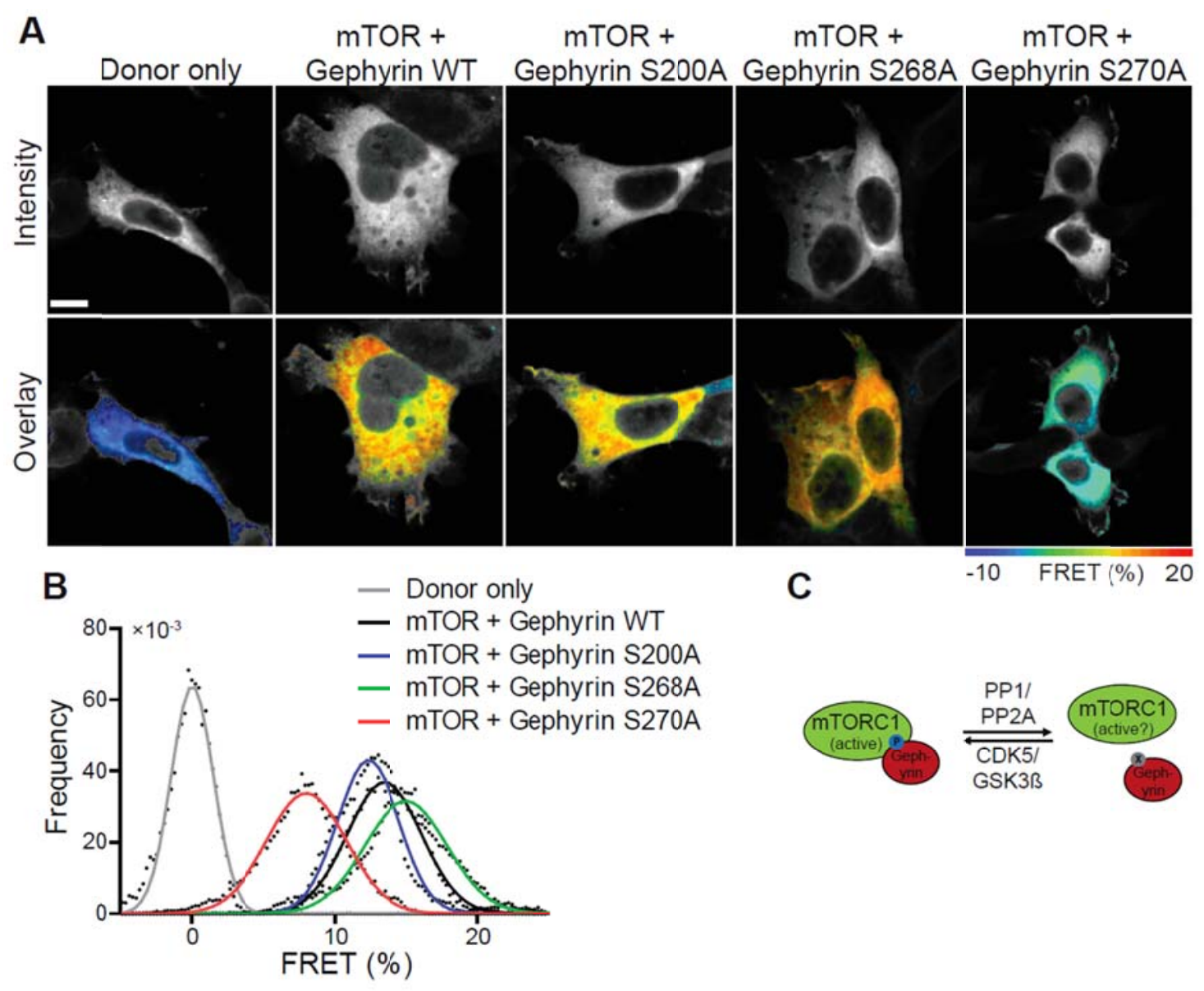

Figure 24: mTOR gephyrin interaction is dependent on gephyrin pS270

A: Representative intensity (top row) (grayscale) and FRET efficiency images overlayed with the corresponding intensity images (bottom row) (false colour) of EGFP-mTOR and mutants of mCherry-gephyrin, coexpressed in HEK29 cells and stained for the C-terminus of mTOR with 7C10 mTOR antibody labelled with ATTO 580Q. Scale bar $10 \mu \mathrm{m}$. B: FRET efficiency distributions per condition are shown, $n=5$. C: Schematic description of the regulation of the mTOR-gephyrin interaction. 


\subsection{FRET-based analysis of gephyrin clustering}

Gephyrin is the major scaffolding molecule of the inhibitory postsynapse and thought to cluster in a highly ordered process via its G- and E-domains. The G-domains form trimers while the E-domains form dimers. Both processes together give rise to a hexagonal lattice structure which is thought to organise the inhibitory postsynaptic membrane. Most studies on gephyrin cluster formation measure the interaction of isolated single $\mathrm{G}$ or $\mathrm{E}$ domains that were expressed in E.coli. However, eukaryotic proteins expressed in bacterial expression systems lack all post-translational modifications due to the absence of the enzyme machinery exerting these modifications. Gephyrin is known to be a highly phosphorylated protein and its aggregation process seems to be regulated by post-translational modifications and involves the interaction of the G-, E- and C-domain. It is therefore indicated to study the clustering of full length gephyrin in eukaryotic cells, preferably in neurons.

The analysis of oriented interaction events is a forté of FRET assays due to its extreme distance dependence.

The oriented clustering of gephyrin was analysed by two separate FRET assays, the first for the trimerisation of the $G$ domains and the second for the dimerisation of $E$ domains. For the trimerisation assay, N-terminally labelled EGFP-gephyrin was coexpressed with with $\mathrm{N}$-terminally labelled mCherry-gephyrin in a 1:3 molar ratio and FRET was measured by determining the lifetime of EGFP by TCSPC-FLIM. Only cells without visible gephyrin aggregates were selected for imaging. Small aggregates were thresholded by their high intensity.

Upon co-expression with mCherry-gephyrin, the lifetime of EGFP-gephyrin was significantly reduced due to FRET (Figure 25). The FRET efficiency was $5.6 \%$ (SD: $1.6 \%)$. 
A
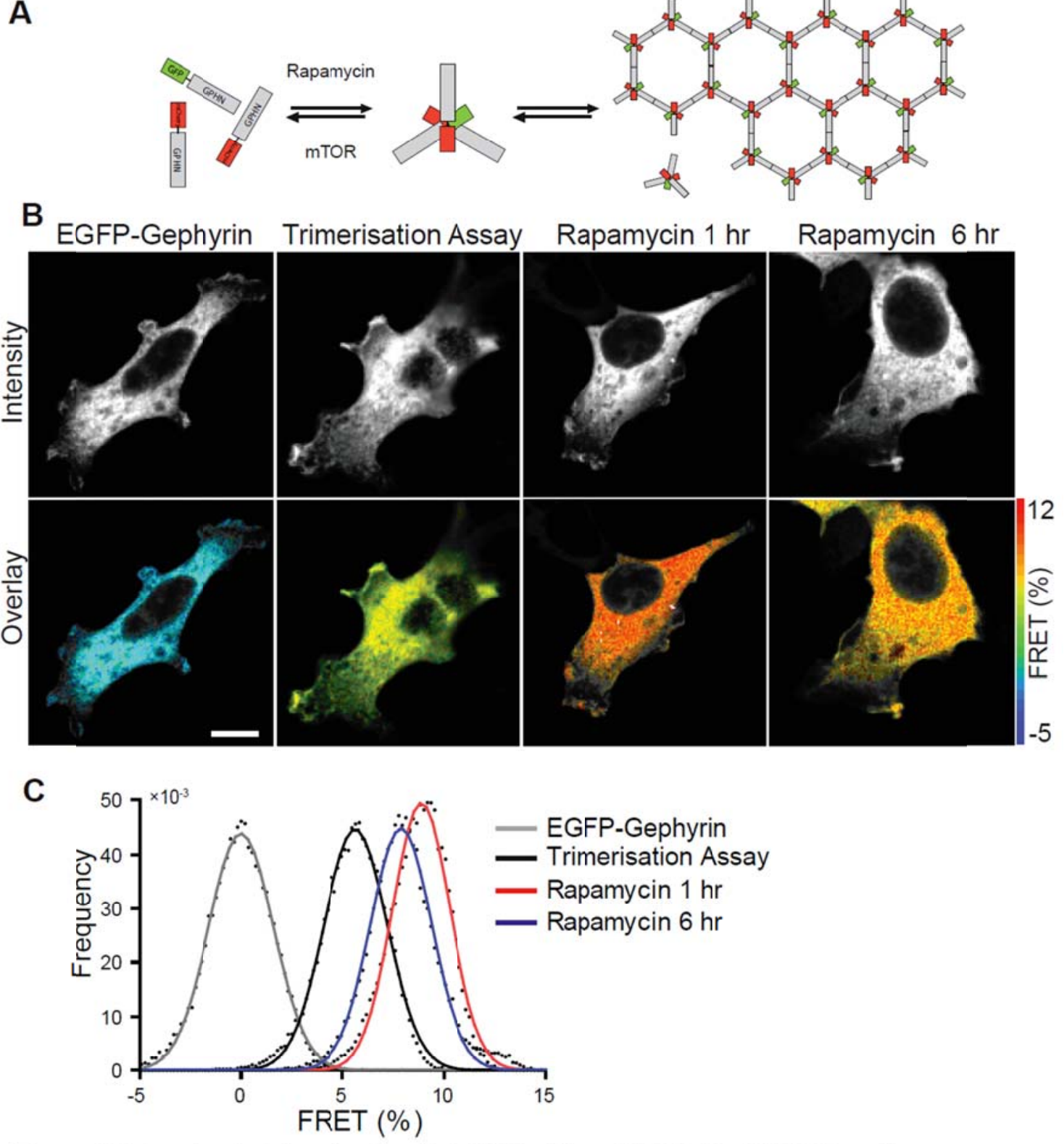

Figure 25: Gephyrin trimerisation is inhibited by mTORC1 in HEK293 cells

A: Schematic representation of the assay principle, regulatory mechanism and clustering process of gephyrin. B: Representative intensity (top row) (grayscale) and FRET efficiency images overlayed with the corresponding intensity images (bottom row) (false colour) of EGFP-gephyrin and mCherry-gephyrin, coexpressed in HEK293 cells. Scale bar $10 \mu \mathrm{m}$. C: FRET efficiency distributions per condition are shown, $n=5$. 
From the previous experiments analysing the interaction of mTOR and gephyrin, it was known that gephyrin interacts with active mTORC1. It was therefore tested if mTORC1 inhibition had any effect on gephyrin trimerisation. The FRET efficiency increased upon mTORC1 inhibition with rapamycin to $8.9 \%$ (SD: $1.4 \%$ ) which is an increase of nearly $60 \%$, indicating that $\mathrm{mTORC} 1$ interaction with gephyrin exerts a strong negative effect on gephyrin clustering.

Next, it was next tested how clustering-impaired mutants of gephyrin influence the formation of gephyrin trimers. To this end, the trimerisation-impaired mutant ' $4 \times R^{\prime}$ and the dimerization-impaired mutant 'RER', were tested for there effect on trimerisation. In the $4 \times \mathrm{R}$ mutant, four hydrophobic residues located at the trimerisation interface are replaced by $R$ (F90R, L113R, L128R and L168R) which results in reduced trimer formation. In the optical gephyrin trimerisation assay, this mutant strongly impeded trimer formation when expressed together with WT mCherry-gephyrin (WT: $6.8 \%$, SD: $2.0 \%$; 4×R: $2.1 \%$, SD: $2.0 \%$ ). In the dimerisation-mutant RER, three amino acids within the dimerisation interface were replaced by arginine or glutamic acid residues (G483R, R523E, A532R). Interestingly, the dimerisation-impaired mutant impeded the trimerisation as well, since the measured FRET efficiencies were reduced to $3.5 \%$ (SD: $2.3 \%$ ). This result could mean two things, either the E-domain is involved in the trimerisation of the Gdomains or, there is connection between interdependent pools of gephyrin monomers, trimers and the lattice. If the lattice formation is impeded, high concentrations of trimers favour the disassembly into gephyrin monomers like in coupled equilibrium reactions. This result also answers the question about the state that gephyrin is in when expressed in HEK293 cells. It apparently forms the hexagonal lattice spontaneously and without the facilitation by synaptic adhesion molecules, as otherwise the dimerisation mutant would have an effect on FRET (Figure 26). 
A

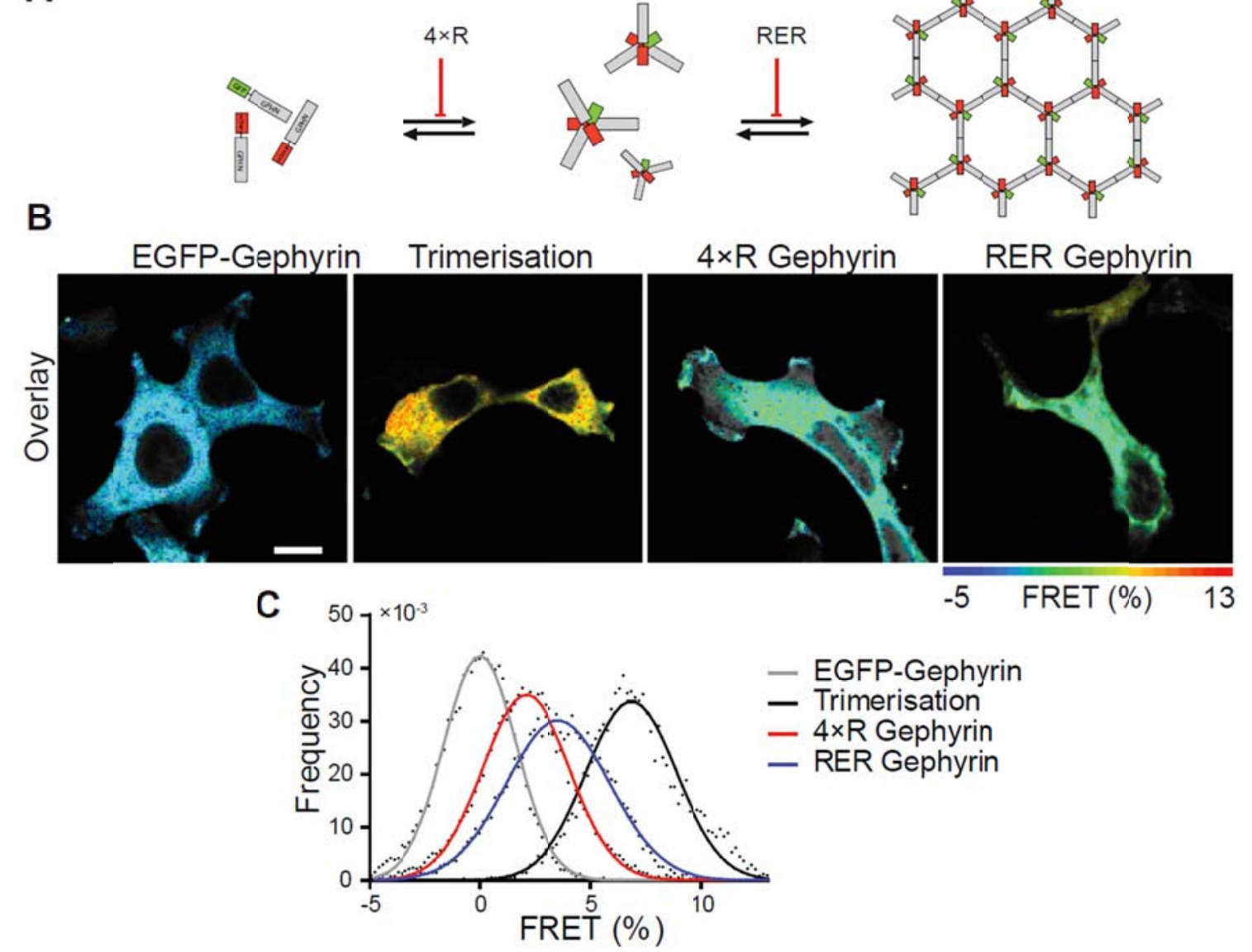

Figure 26: Gephyrin mutants reduce clustering

A: Schematic representation of the assay principle, clustering process and effect of mutantions. B: Representative FRET efficiency images overlayed with the corresponding intensity images of clustering impaired mutants of EGFP-gephyrin, coexpressed with WT mCherrygephyrin. Scale bar: $10 \mu \mathrm{m}$. C: FRET efficiency distributions of images per condition are shown, $\mathrm{n}=5$.

It was next tested if the trimerisation of gephyrin is facilitated by its major structural interaction partner neuroligin 2 and collybistin. Whether collybistin and neuroligin 2 only recruit gephyrin to the postsynaptic membrane or if they are able to catalyze the clustering process itself, is not known. To answer this question, neuroligin 2 or a constitutively active form of collybistin 2 (-SH3) were co-expressed with the trimerisation assay and detected by antibody staining. Upon co-expression with constitutively active collybistin, gephyrin shows an increased membrane localisation as well as increased clustering. In areas where high collybistin signals coincide with high gephyrin signals, the measured FRET efficiencies where up to $20 \%$ and the mean FRET efficiency was increased to $13 \%$ (SD: $5.8 \%$ ). The co-expression with 
neuroligin 2 induced the formation of spot-like gephyrin structures that appeared to be localized to the plasma membrane. These gephyrin spots exhibit high FRET efficiencies of up to $16 \%$ and colocalised with neuroligin 2 spots (Figure 27).

The strong induction of gephyrin clustering by collybistin and neurologin 2 suggests that both molecules activate gephyrin clustering beyond the recruitment to the plasma membrane.

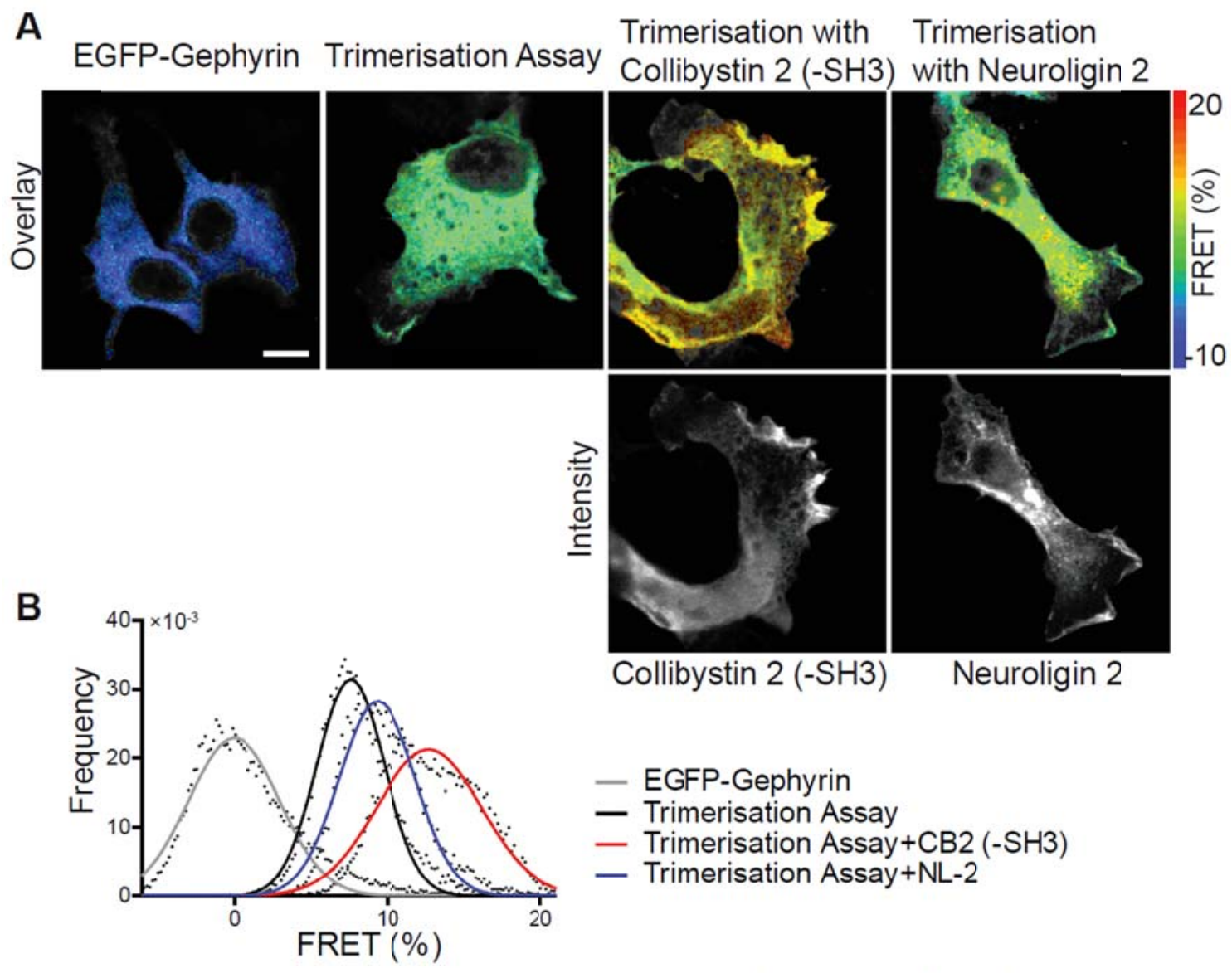

Figure 27: Gephyrin trimerisation promoted by Collybistin 2 (-SH3) and Neuroligin 2

A: Representative FRET efficiency images overlayed with the corresponding intensity images of EGFP-gephyrin and mCherry-gephyrin, coexpressed with constituitively active collybistin 2 (CB2 $-\mathrm{SH} 3$ ) or neuroligin 2 are shown (top row). Collybistin 2 and neuroligin 2 were stained with Alexa 647 (bottom row) (grayscale) Scale bar: $10 \mu \mathrm{m}$. B: FRET efficiency distributions per condition are shown, $n=5$. 
The formation of the hexagonal lattice from trimeric building blocks involves the dimerisation of E-domains. To analyse this process, C-terminally labelled mTurquoise- and EYFP-fusion constructs of gephyrin where generated and coexpressed at a 1:2 molar ratio. The localization pattern of gephyrin-mTurquoise was similar to that of EGFP-gephyrin. The measured mean FRET efficiency between gephyrin-mTurquoise and gephyrin-EYFP was $4.4 \%$ (SD: $2.6 \%$ ). When coexpressed with constitutively active CB2 or NL2 two FRET efficiencies more than doubled to $11.5 \%$ (SD: $4.0 \%$ ) or $11.1 \%$ (SD: $3.1 \%$ ) with CB2or NL2 respectively. This dramatic increase of $161 \%$ suggests that dimerisation of gephyrin E-domains is a regulated process that is facilitated by CB2 and NL2 (Figure 28). 
A
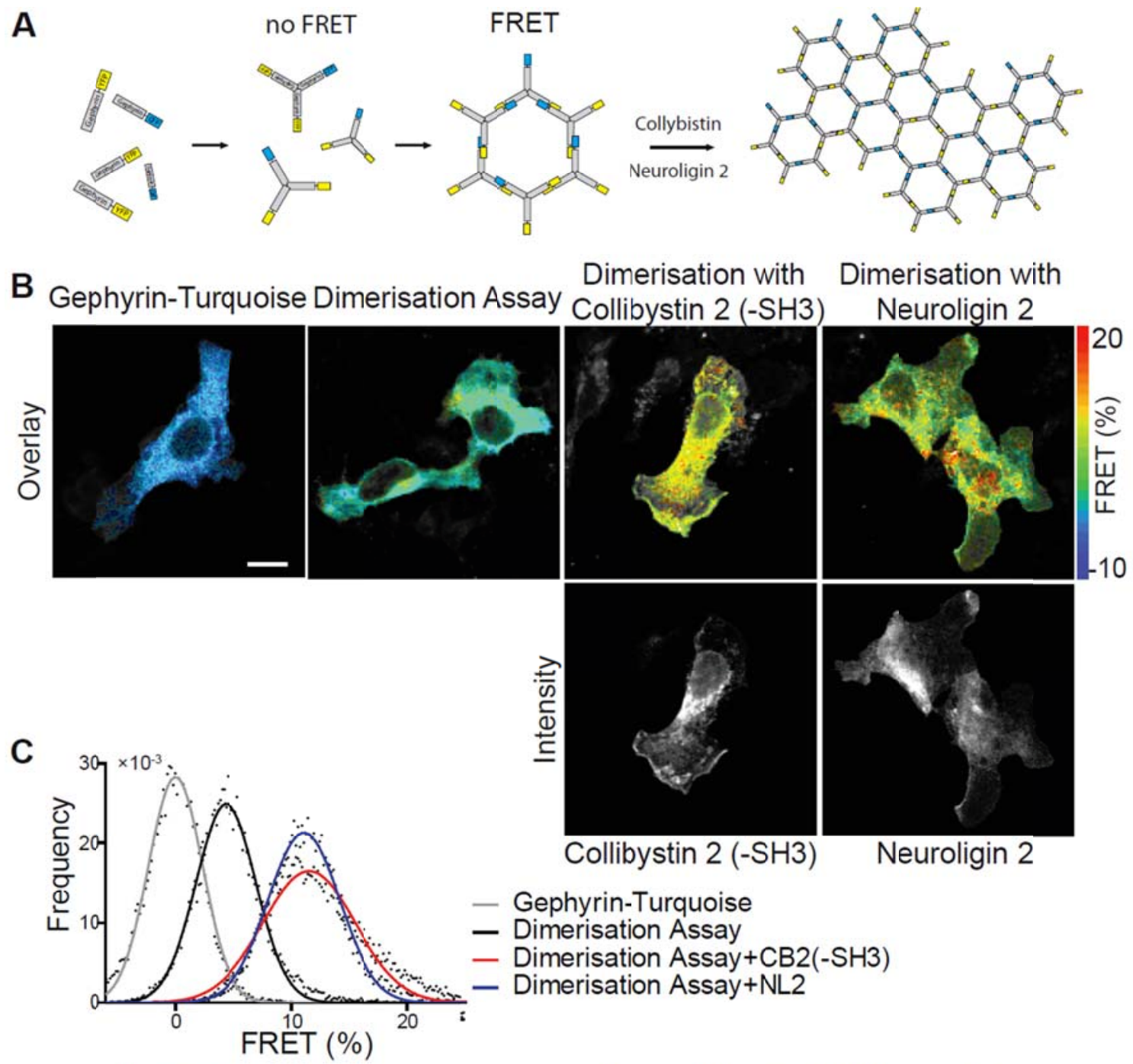

Figure 28: Gephyrin dimerisation promoted by Collybistin (-SH3) and Neuroligin 2 A: Schematic representation of gephyrin clustering analysed by C-terminally labelled gephyrin-mTurquoise and -EYFP. B: Representative FRET efficiency images overlayed with the corresponding intensity image of gephyrin-mTurquoise and gephyrin-EYFP, coexpressed with constituitively active collybistin 2 (CB2-SH3) or neuroligin 2 (top row). Intensity images of Collybistin 2 (-SH3) and neuroliging 2, stained with Alexa 647, are shown (bottom row) (grayscale). Scale bar: $10 \mu \mathrm{m}$. C: FRET efficiency distributions per condition are shown, $\mathrm{n}=5$. 


\subsection{Gephyrin trimerisation in GABAergic postsynapses}

Gephyrin clustering seems to be a regulated process which depends on the molecular composition of the inhibitory post-synapse. Among the determinants, subunit compositions of $\mathrm{GABA}_{A}$-receptors, as well as the abundance of CB2 and NL2, were identified to play a role. It was therefore tested if gephyrin clustering could be measured in GABA-ergic postsynapses. To this end, EGFP- and mCherrygephyrin were co-expressed in hippocampal rat neurons which were stained with an antibody directed against the $\gamma_{2}$ subunit of the $G_{A B A}$-receptor. $\gamma_{2}$ subunit-containing $\mathrm{GABA}_{\mathrm{A}}$-receptors do not show extra-synaptic localisation so that they can be used to distinguish synaptic and extra-synaptic gephyrin clusters. The signal from y2 subunitcontaining $\mathrm{GABA}_{\mathrm{A}}$-receptors was used to create an optical mask that was used to exclude extra-synaptic gephyrin clusters from the analysis. The results show that by combining the gephyrin clustering assay with markers of inhibitory postsynapses, gephyrin clustering can be measured at identified inhibitory postsynapses. As observed in HEK293, cortical neurons treated with rapamycin show an increase in FRET efficiencies by $67 \%$ from $3.7 \%$ (SD: $2.3 \%$ ) to $6.2 \%$ (SD: $2.2 \%$ ). Since there is no ectopically expressed mTOR present in these neurons, gephyrin must have interacted with endogenous $\mathrm{mTORC} 1$ which was subsequently inhibited by the administration of rapamycin (Figure 29). 
A
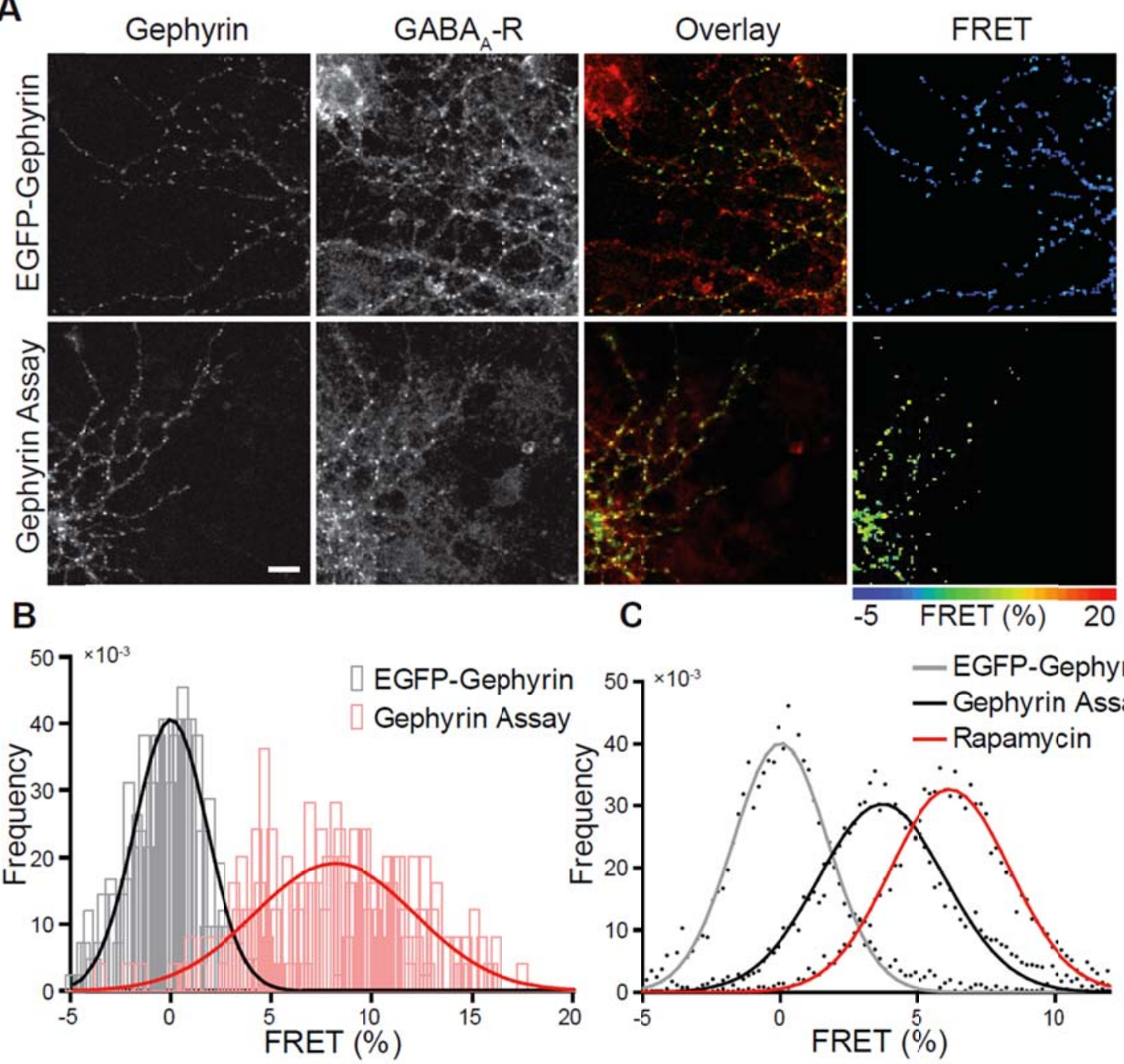

C

- EGFP-Gephyrin

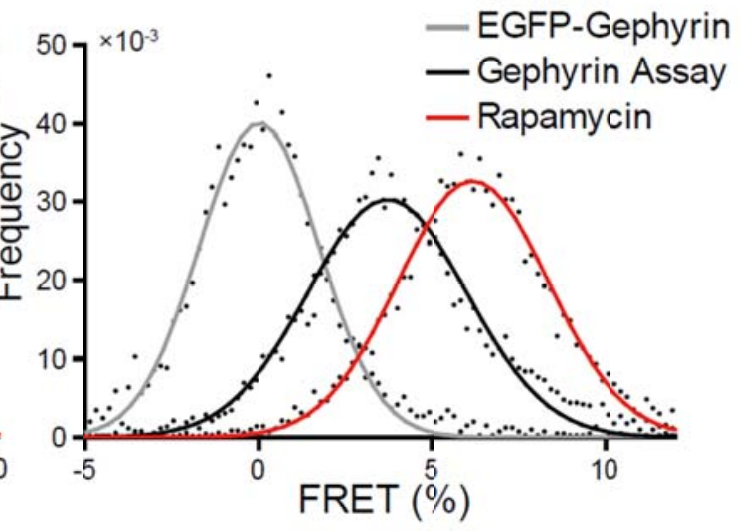

Figure 29: Gephyrin trimerisation at GABAergic synapses is inhibited by mTORC1

A: Representative intensity images of EGFP-gephyrin, GABA $_{A}$-receptors, overlay of gephyrin and GABA $_{A}$-receptors and FRET images are shown. Rat Coritcal neurons (DIV16) coexpressing $\mathrm{N}$-terminally labelled GFP-and $\mathrm{mCherry}$-gephyrin were treated with rapamycin and stained for $\gamma 2$ subunit of $G_{A B A}$-receptor. The $G_{A B A}$-receptor staining was used to mask out extrasynaptic gephyrin clusters. Scale bar: $10 \mu \mathrm{m}$. B: FRET efficiency distributions of masked $\mathrm{GABA}_{A}$-receptor positive gephyrin clusters. C: Gephyrin trimerisation is promoted by $\mathrm{mTORC} 1$ inhibition following treatment with rapamycin. 


\section{Discussion}

\subsection{FRET-based mTORC1 and mTORC2 activity assays}

$\mathrm{mTOR}$ is a serine/threonine kinase that forms two functionally and structurally distinct complexes, mTORC1 and mTORC2. mTORC1 regulates mRNA translation, lipid biosynthesis and cellular growth, while mTORC2 regulates cytoskeletal rearrangements and cellular survival.

During activation of mTORC1, mTOR is phosphorylated at serin 2448 by S6K1. This phosphorylation is a positive feedback loop that keeps mTORC1 activated by its downstream target, which sustains mTORC1 activity. It serves as a marker of active mTORC 1 in the optical assay presented here.

Active mTORC2 phosphorylates Akt at S473. This phosphorylation event is executed exclusively by mTORC2 and was therefore used as marker in the mTORC2 activity assay presented here.

Phosphorylation events are fast post-translational modifications that have the capacity to modulate the signalling properties of molecules. They can be assayed by phosphorylation-specific antibodies.

In this study, two FRET-based phosphorylation sensors were established that allow the quantification of endogenous mTORC1 and mTORC2 activity at the single cell level. Antibodies might exhibit unspecific binding, which could disguise a physiological effect and result in miss-interpretation.

The major challeng in creating these assays, was the choice of the right antibodies. In this case it was even more difficult because 'good' antibodies were only available from rabbit. This problem is more frequent than expected: the search engine www.biocompare.com will find a total number of 1,742,975 antibodies generated in rabbit, but only 709,700 antibodies generated in mouse. Having to use two antibodies from the same species is therefore a frequent obstacle for researchers.

The use of two antibodies from the same species comes with the problem of high cross-reactivity (Figure 8 ). In the present study problems caused by cross-reactivity could in part be eased by the use of a monovalent blocking antibody. Monovalent Fab fragments reduced the measured standard deviations by up to $50 \%$, which improved the accuracy of the assays (Figure 10). 
Antigen trapping was identified as the major source of cross-reactivity when using two antibodies from the same species (Figure 15). Strategies to overcome this problem included blocking of unsaturated antigen binding domains by monovalent Fab fragments or the use of blocking peptides but all of these strategies were dropped due to practical issues that come along with the use of additional antibodies, like epitope loss due to extensive washing, time consumption of repeated antibody incubations and steric problems.

We identified the use of fluorescently labelled, monovalent Fab fragments as secondary antibodies to be the only practical way to solve this problem.

\subsection{FRET optimisations}

In time correlated single photon counting, even small differences in FRET efficiencies can be resolved, as long as there are enough photons to ensure a proper fitting of the fluorescence lifetime data. This was ensured by replacing Cy3, which exhibits a comparably low brightness and high bleaching susceptibility, with a brighter and more photostabile fluorophore ATTO 532. In combination with an improved FRETpartner, like ATTO 580Q, the FRET efficiency was maximized (Figures 11 and 12).

The Förster distance is larger between fluorophores with longer emission wavelengths as the overlap integral of donor emission and acceptor absorption factors in the wavelength by the power of 4 (see section 1.7.1). It is therefore beneficial to choose dyes in the $500-600 \mathrm{~nm}$ or the $600-700 \mathrm{~nm}$ spectral range. For practical reasons, the donor fluorophore should be in the visible range. Therefore the $500-600 \mathrm{~nm}$ spectral range was chosen as the donor.

The present study focused on dyes from the company ATTO-TEC (Berlin, Germany) as the most detailed information relevant for FRET and TCSPC FLIM are available for these dyes. The Förster distance for every combination of two ATTO-TEC dyes, bleaching curves, absorption and emission spectra, fluorescence quantum yields and other valuable data are available.

ATTO 532 in combination with ATTO $580 Q$ has a calculated Förster distance of $67 \AA$. In contrast, Cy 3 and Cy5 have a Förster distance of $54 \AA$, an improvement of $24 \%$.

The imaging set up used in the present study is equipped with a $532 \mathrm{~nm}$ pulsed laser diode. Suitable dyes for this excitation are ATTO 532 and ATTO 550. A direct 
comparison of ATTO 532 and ATTO 550 with Cy3 shows improvements in terms of bleaching, quantum yield and fluorescence lifetime. ATTO 550 is slightly superior in terms of absorption but ATTO 532 has the better in fluorescence quantum yield and the longer lifetime. However, both dyes are clearly more photostabile than Cy3, which is the most important improvement.

The assay was constructed with the aim of combining it with molecular markers of cellular identity. Therefore, the dark fluorescence quencher ATTO 580Q was used as an acceptor for FRET.

In terms of FRET, the assay as it currently is can be considered optimised. There are only few adjustments left like increasing the labeling density (currently 0.97 and 1.17 labels per Fab fragment) of the Fab fragments. But this must be reviewed carefully because over-labelling might interfere with the affinity due to blocking of the antigen binding domain or with antibody solubility. ATTO $580 \mathrm{Q}$ for instance is very hydrophobic.

\subsection{The mTORC1 assay}

After antigen trapping was excluded by the use of monovalent Fab fragments, the primary antibodies were evaluated in an iterative process. In Figure 15 it was shown that both antibodies have different staining patterns. A prominent nuclear signal was observed with the abcam antibody, while the 7C10 showed a granular staining pattern. In the FRET assay, both antibodies showed moderate FRET efficiencies. Rapamycin treatment revealed that the high FRET efficiencies in the nucleus were probably due to cross-reactivity.

The experiment that compared the IHC-specific antibody against the XP demonstrated how cross-reactivity hampers the dynamic range of the assay. The IHC-specific showed mean FRET efficiency nearly twice as high as the XP. However, the high residual FRET under rapamycin conditions of $6 \%$ left a true dynamic range of only $3 \%$, combined with a wider standard deviation due to higher variability. The $\mathrm{XP}$ antibody was therefore considered superior, despite the fact that the measured FRET efficiencies were rather low (Figures 16 and 17).

Another marker of mTORC1 activity that was tried out here was the phosphorylation of S6K1 at T389. This assay did not fly due to bad antibody quality. 


\section{4 mTORC2 assay}

The mTORC2 assay designed was based on the principles of the mTORC1 assay. The measured FRET efficiencies reflected changes in the biological substrate. The assay showed that inhibition of upstream activator of the Akt pathway lead to a complete abrogation of phosphorylation, while insulin increased Akt phosphorylation (Figure 20).

The effect of chronic rapamycin treatment on mTOC2 activity was tested in HEK293 cells (Figure 21). Chronic treatment with rapamycin induced partial disruption of mTORC2 in a previous report (Sarbassov et al., 2006). The disruption of mTORC2 renders cells insensitive to insulin. In fact, rapamycin induced insulin resistance is the major obstacle that has prevented the use of rapamycin in the clinical therapy of cancer, neurodegenerative disorders, cardiovascular disease and other age-related diseases. The data presented here shows that mTORC2 is resistant to chronic rapamycin treatment in HEK293 cells. Instead, mTORC2 signalling is increased by chronic rapamycin exposure. This increase may be due to a negative feedback mechanism downstream of S6K1. Active S6K1 phosphorylates IRS and thereby primes it for proteasomal degradation. This way S6K1 activity limits the activation of mTORC2. Rapamycin disrupts this feedback mechanism, leading to an accumulation of IRS which fuels the activation path of mTORC2.

In 2012, Lamming and colleagues showed that rapamycin-induced insulin resistance was mediated rapamycin-sensitive mTORC2. The mechanism underlying this phenomenon was described by Schreiber and colleagues (2015). They identified the ratio of FKBP12 to FKBP51 as the determining factor for mTORC2 rapamycin resistance. Cells with a low ratio like HELA or HEK293 are rapamycin insensitive while cells with a high ratio like $\mathrm{PC} 3$ or $\mathrm{C} 2 \mathrm{C} 12$ are rapamycin sensitive.

\subsection{Compromised mTORC1 and mTORC2 signalling in Rett syndrome.}

The mTORC1- and mTORC2 activity assays consistently reported reduced mTORC1 and $\mathrm{mTORC} 2$ activities in neuronal cells derived from MeCP2-KO mice. The reduced 
mTORC1 activity seemed to be restricted to neurons while the reduced mTORC2 activity was observed in all neuronal cell types (Figure 18 and 22).

The reduced activity of $\mathrm{mTORC} 1$ is in agreement with a report from Ricchiardi and colleagues (2011) that showed reduced activation of S6K1 in brain lysates of MeCP2-KO mice.

The observed cell-type specific reduction of mTORC1 activity awaits confirmation by multiplexing the current FRET assay with markers of neuronal identity. This can be done as the assay was designed with application this in mind. As the acceptor is a quencher, it allows the re-use of the acceptor channel for markers of neuronal identity. These could be NeuN, a neuron marker. Moreover, excitatory neurons can be identified by vesicular glutamate transporter 1 or 2 , while inhibitory neurons can be identified by vesicular inhibitory amino acid transporter or glutamate decarboxylase 65.

A genetic link between MeCP2 and mTOR signalling was elucidated very recently. Tsujimura and colleagues (2015), describe the interaction between MeCP2 and the microRNA microprocessor drosha complex. This interaction regulates the processing of specific miRNA-199a which targets Hif1a, Sirt1 and Pde4d. These genes code inhibitory factors of the mTOR pathway, HIF1a, SIRT1 and PDE4D, which are upregulated in the brain of MeCP2-KO mice. Loss of miR-199a results in decreased mTOR activity in the brain and recapitulated RTT phenotypes in vivo. Vice versa, expression of miR-199a rescues neuronal abnormalities caused by the loss of MeCP2.

MeCP2-KO also shows reduced BDNF expression. BDNF is known to sustain mTORC1 signalling by activating the TrkB receptor/Akt/mTOR signalling axis in neurons. MeCP2-KO mice show reduced expression, transport and secretion of BDNF. Bdnf-KO mice recapitulate many phenotypes observed in the MeCP2-KO mice.

Reduced activation of TrkB may also explain reduced mTORC2 activity since this is a downstream event. This might represent a positive feedback mechanism, missing in MeCP2-KO mice.

During its activation, Akt is recruited to the plasma membrane where it is phosphorylated at threonine 308 by PDK1. This phosphorylation is a pre-requisite for the mTORC2-dependent phosphorylation of Akt at S473. Since the activation 
process of mTORC2 is unknown, it is not clear if the reduced S473 phosphorylation is due to a reduced mTORC2 activity or due to the reduced availability Akt phosphorylated at T308. mTORC2 might be active, but missing a substrate to phosphorylate. This could be interpreted incorrectly as a reduced mTORC2 activity. A more direct readout of $\mathrm{mTORC2}$ activity may be the mTOR-Rictor interaction which was tried but did not yield any dynamic changes in FRET efficiencies.

Other tested parameters included the interaction between mTORC2 and the TSC1/TSC2 complex, the phosphorylation of tuberin at S939 or T1462, and the phosphorylation of downstream target PKC alpha at S657, with similar results.

\section{6 mTOR-gephyrin interaction}

In this study the interaction between EGFP-mTOR and mCherry-gephyrin was observed in HEK293 cells (Figure 23). It was found that the interaction was dependent on active mTOR within MTORC1, which could be disrupted by rapamycin or mutation of S270 within gephyrin (Figure 24).

The little amount of literature on mTOR-gephyrin interaction is contradictory (Sabatini et al., 1999; Wuchter et al., 2012). The mTOR-gephyrin interaction was first described by Sabatini and colleagues (1999). Gephyrin was found to interact with mTOR's gephyrin binding domain (GDB), a stretch of amino acids that show $45 \%$ sequence identity with the $\beta$-loop of glycine receptor. Mutations in the GDB disrupted the interaction with gephyrin and mTOR failed to activate S6K and 4E-BP1. The data suggests that gephyrin binds to active mTOR within mTORC1 and that this interaction is necessary for proper induction of protein-translation.

The data presented here is in line with this study and confirms the interaction of gephyrin with active mTORC1.

In contrast, Wuchter and colleagues described the exact opposite mechanism. They find that in neurons, gephyrin binds to inactive mTOR and that the activation of mTORC1 by BDNF, releases gephyrin from mTOR and allows for it to cluster. The authors show that mTORC1 activation increases gephyrin cluster density while mTORC1 inhibition decreases gephyrin cluster density. On the functional level, mTORC1 inhibition reduced GABAergic inhibition within the neuronal network, which resembled the effect of $\mathrm{GABA}_{\mathrm{A}}$ receptor inhibition. 
A potential reason for this discrepancy might be the overexpression of mTOR and gephyrin. The resulting unnaturally high concentrations of both proteins might induce the observed interaction.

Nevertheless, it should be tested if the interaction between mTOR and gephyrin can be observed in neurons as well.

The inhibition of endogenous mTORC1 in primary cortical neurons increased gephyrin clustering as measured with the gephyrin trimerisation assay (Figure 29). This suggests that gephyrin clustering was impeded by the interaction with endogenous active $\mathrm{mTORC} 1$ and that mTORC1 inhibition enabled gephyrin clustering. This experiment is in line with the observations made here and with Sabatini's data. It suggests that endogenous active mTORC1 interacts with gephyrin to negatively regulate it's clustering in primary neurons.

The mTOR-gephyrin interaction assay was used to find gephyrin-mutants that exhibit an altered interaction with mTOR. Serine 270 was identified as an important determinant of this interaction. The regulation of the mTOR-gephyrin interaction by phosphorylation within gephyrin represents an intriguing regulatory mechanism. It would allow for a fast and localized protein translation at the inhibitory postsynapse. It would be interesting to know, which clustering-state of gephyrin is preferred by mTOR to interact with. To this end, the mTOR-gephyrin interaction should be analysed for the clustering impaired mutants of gephyrin.

Gephyrin S270 was described to be phosphorylated by GSK-3 $\beta$ and CDK5 (Tyagarajan et al., 2011; Kuhse et al., 2012). The alanine exchange mutation resulted in the formation of more and larger gephyrin clusters accompanied by increased amplitude and frequency of mIPSCs. GSK-3 $\beta$ activity is negatively regulated by Akt activity. Under culture conditions, growth factors and cytokines are provided in excess and Akt signalling is close to its maximum. The activity of GSK-3 $\beta$ should therefore be close to its minimum and hence gephyrin should be unphosphorylated. However, the S270A mutation had a substantial effect on gephyrin clustering. It is therefore likely that CDK5 primarily exerts phosphorylation at S270A under culture conditions.

The inhibition of CDK5 reduces the stability of gephyrin clusters as well as $\mathrm{GABA}_{\mathrm{A}}$ receptor clusters (Kalbouneh et al., 2014). This finding points towards major discrepancy in the effect of GSK-3 $\beta$ and CDK5 at S270. The inhibition of GSK-3 $\beta$ as 
well as the expression of S270A increased gephyrin cluster size (Tyagarayan et al., 2011), but the pharmacological inhibition of CDK5 decreases the size of gephyrin and $\mathrm{GABA}_{\mathrm{A}}$ receptor clusters. One possible explanation could be that other kinases phosphorylate the regulatory domain of gephyrin, and that different combinatorial patterns of phosphorylation have divergent functional consequences.

\subsection{Gephyrin clustering measured by FRET}

\subsubsection{Gephyrin trimerisation}

In order to analyse the clustering of gephyrin, N-terminal fusion constructs of EGFPgephyrin and mCherry-gephyrin were co-expressed in HEK293. The trimerisation of full length gephyrin could be measured by FRET between EGFP and mCherry (Figure 25).

The co-expression of CB2 or NL2, recruited gephyrin to the plasma membrane and promoted trimer formation (Figure 27), which resulted in markedly increased FRET efficiencies. Collybistin seemed to be more efficient in promoting gephyrin trimerisation. Also rapamycin induced gephyrin trimerisation in HEK293 as well as in primary cortical neurons (Figure 29).

\subsubsection{Gephyrin trimerisation and dimerization mutants}

The expression of dimerisation and trimerisation impaired mutants of gephyrin allowed structural insights into gephyrin clustering in HEK293 cells. The trimerisation impaired mutant $4 \times \mathrm{R}$ reduced the trimerisation by $71 \%$ which was expected. Unexpectedly the dimerisation impaired mutant RER was also efficiently impaired in trimerisation, by remarkable $57 \%$. This was surprising because of structural data. Nevertheless, the regulatory $\mathrm{C}$-domain of gephyrin might allow for a direct interaction of the G- and the E-domain even though a direct interaction has not been described yet. It is more likely that gephyrin monomers, trimers and the hexagonal lattice form three interdependent pools that are in a state of dynamic equilibrium (Figure 26). In this model, all three pools communicate via concentration. A very high concentration of monomers would promote the trimerisation process. Vice versa, the massive depolymerisation of the lattice into trimers would also promote the disintegration of 
trimers into monomers. This was observed in the dimerisation mutant. The dimerisation impaired mutant showed markedly reduced trimerisation, which might be explained by the fact that the formation of the lattice is prohibited and the expected high timer concentration might cause the disintegration of trimers into monomers.

\subsubsection{Gephyrin dimerisation}

C-terminal fusion constructs of full length gephyrin with either mTurquoise or EYFP were used to quantify the dimerisation of gephyrin's E-domains in HEK293 cells. Like in the trimerisation assay collybistin and neuroligin 2 are potent activators of the dimerisation (Figure 29). This is in line with the previous assumption of inter pools of gephyrin clustering states that communicate in a dynamic equilibrium. A massive increase in trimer-formation would also drive the dimerisation of the E-domains.

Unfortunately, our assay as it was designed here was not able to distinguish between the hexagonal lattice model and the stacked trimer model (Figure 1). In both models the E-domains as well as the G-domains interact in a way that allows for FRET to occur. The analysis of $4 \times \mathrm{R}$ and the RER mutant in the dimerisation assay could help to elucidate the clustering process further. Also the measurement of diffusion constants of gephyrin clustering mutants with fluorescence correlation spectroscopy could help to answer the final questions about the correct clustering model.

Both gephyrin assays, for the first time allow a quantification of the oligomerisation process of full length gephyrin at the inhibitory post-synapse. This represents a clear improvement over the so far used analysis of purified single domains that are expressed in heterologous expression systems like E.coli. Bacterial expression systems do not possess the enzymatic machinery to exert post-translational modifications at eukaryotic proteins. The assays designed here represent new optical tool to analyse effects of post-translational modifications on gephyrin clustering. 


\section{Conclusions and Future Perspectives}

In this study antibody-based FRET assays of mTORC1 and mTORC2 activity were established and evaluated. They have shown to work on the single cell level and across species. They provide qualitative and quantitative information on mTORC1 and mTORC2 activity and are designed to be combined with molecular markers of cellular identity or of sub-cellular structures.

They were used to confirm the reduced mTORC1 activity in neurons of MeCP2-KO mice, a mouse model of the Rett syndrome. These experiments suggested a neuronspecific reduction in $\mathrm{mTORC} 1$ signalling. This finding needs confirmation by multiplexing the mTORC1 assay with neuronal markers. The identification of affected neuronal subtypes might help to find therapeutic strategies to ease symptoms of Rett syndrome in the future.

The interaction of mTOR with gephyrin, a molecular organiser of inhibitory postsynapses, represents a potential mechanism for inhibitory synapse formation and plasticity. To further elucidate the details of this interaction a FRET assay was established that confirmed the interaction in HEK293 cells. Mutations of gephyrin that corrupt the interaction were searched and S270 was identified as an important determinant of this interaction.

In the future the mTOR-gephyrin interaction and the consequences of its failure should be confirmed at the inhibitory post-synapse. Moreover the meaning of this interaction in the context of synapse formation and plasticity should be elucidated.

The functions of gephyrin at the inhibitory postsynapse include the organisation of inhibitory neurotransmitter receptors and the integration of neuron-specific signalling. The organisation of neurotransmitters requires gephyrin to build a 2 dimensional network underneath the synaptic membrane. Gephyrin clustering assays were established that allowed the quantification of that process. In the future these assay can be used to determine how clustering is connected to the signalling function of gephyrin and what the consequences of these interconnected functions are. 


\section{References}

Abe N, Borson SH, Gambello MJ, et al (2010) Mammalian Target of Rapamycin (mTOR) Activation Increases Axonal Growth Capacity of Injured Peripheral Nerves. J Biol Chem 285:28034-28043. doi: 10.1074/jbc.M110.125336

Agronskaia AV, Tertoolen L, Gerritsen HC (2003) High frame rate fluorescence lifetime imaging. J Phys -Appl Phys 36:1655-1662.

Ai H, Hazelwood KL, Davidson MW, Campbell RE (2008) Fluorescent protein FRET pairs for ratiometric imaging of dual biosensors. Nat Methods 5:401-403. doi: 10.1038/nmeth.1207

Akrap N, Seidel T, Barisas BG (2010) Förster distances for fluorescence resonant energy transfer between mCherry and other visible fluorescent proteins. Anal Biochem 402:105-106. doi: 10.1016/j.ab.2010.03.026

Aryal P, Kim K, Park P-H, et al (2014) Baicalein induces autophagic cell death through AMPK/ULK1 activation and downregulation of mTORC1 complex components in human cancer cells. FEBS J 281:4644-4658. doi: 10.1111/febs.12969

Asaka Y, Jugloff DGM, Zhang L, et al (2006) Hippocampal synaptic plasticity is impaired in the Mecp2-null mouse model of Rett syndrome. Neurobiol Dis 21:217-227. doi: 10.1016/j.nbd.2005.07.005

Autry AE, Monteggia LM (2012) Brain-Derived Neurotrophic Factor and Neuropsychiatric Disorders. Pharmacol Rev 64:238-258. doi: 10.1124/pr.111.005108

Ballew RM, Demas JN (1991) Error analysis of the rapid lifetime determination method for single exponential decays with a non-zero baseline. Anal Chim Acta 245:121-127.

Ballew RM, Demas JN (1989) An error analysis of the rapid lifetime determination method for the evaluation of single exponential decays. Anal Chem 61:30-33.

Bar-Peled L, Chantranupong L, Cherniack AD, et al (2013) A Tumor Suppressor Complex with GAP Activity for the Rag GTPases That Signal Amino Acid Sufficiency to mTORC1. Science 340:1100-1106. doi: 10.1126/science.1232044

Barnard EA, Skolnick P, Olsen RW, et al (1998) International Union of Pharmacology. XV. Subtypes of gamma-aminobutyric acidA receptors: classification on the basis of subunit structure and receptor function. Pharmacol Rev 50:291-313.

Bastiaens PI, Squire A (1999) Fluorescence lifetime imaging microscopy: spatial resolution of biochemical processes in the cell. Trends Cell Biol 9:48-52.

Becker EBE, Bonni A (2006) Pin1 Mediates Neural-Specific Activation of the Mitochondrial Apoptotic Machinery. Neuron 49:655-662. doi: 10.1016/j.neuron.2006.01.034

Becker W, Su B, Bergmann A (2009) Fast-acquisition multispectral FLIM by parallel TCSPC. pp 718305-718305-5

Benjamin D, Hall MN (2013) TSC on the peroxisome controls mTORC1. Nat Cell Biol 15:1135-1136. doi: $10.1038 /$ ncb2849

Bercury KK, Dai J, Sachs HH, et al (2014) Conditional Ablation of Raptor or Rictor Has Differential Impact on Oligodendrocyte Differentiation and CNS Myelination. J Neurosci 34:4466-4480. doi: 10.1523/JNEUROSCI.4314-13.2014

Berney C, Danuser G (2003) FRET or no FRET: a quantitative comparison. Biophys J 84:3992-4010. doi: 10.1016/S0006-3495(03)75126-1

Blancquaert S, Wang L, Paternot S, et al (2010) cAMP-Dependent Activation of Mammalian Target of Rapamycin (mTOR) in Thyroid Cells. Implication in Mitogenesis and Activation of CDK4. Mol Endocrinol 24:1453-1468. doi: 10.1210/me.2010-0087 
Blundell J, Tabuchi K, Bolliger MF, et al (2009) Increased anxiety-like behavior in mice lacking the inhibitory synapse cell adhesion molecule neuroligin 2. Genes Brain Behav 8:114-126.

Bockaert J, Marin P (2015) mTOR in Brain Physiology and Pathologies. Physiol Rev 95:1157-1187. doi: 10.1152/physrev.00038.2014

Buckmaster PS, Ingram EA, Wen X (2009) Inhibition of the Mammalian Target of Rapamycin Signaling Pathway Suppresses Dentate Granule Cell Axon Sprouting in a Rodent Model of Temporal Lobe Epilepsy. J Neurosci 29:8259-8269. doi: 10.1523/JNEUROSCI.4179-08.2009

Budreck EC, Scheiffele P (2007) Neuroligin-3 is a neuronal adhesion protein at GABAergic and glutamatergic synapses: Synaptic localization of neuroligin-3. Eur J Neurosci 26:1738-1748. doi: 10.1111/j.1460-9568.2007.05842.x

Cafferkey R, Young PR, McLaughlin MM, et al (1993) Dominant missense mutations in a novel yeast protein related to mammalian phosphatidylinositol 3-kinase and VPS34 abrogate rapamycin cytotoxicity. Mol Cell Biol 13:6012-6023. doi: 10.1128/MCB.13.10.6012

Calamai M, Specht CG, Heller J, et al (2009) Gephyrin Oligomerization Controls GlyR Mobility and Synaptic Clustering. J Neurosci 29:7639-7648. doi: 10.1523/JNEUROSCI.5711-08.2009

Chahrour M, Jung SY, Shaw C, et al (2008) MeCP2, a Key Contributor to Neurological Disease, Activates and Represses Transcription. Science 320:1224-1229. doi: 10.1126/science.1153252

Chang JY, Sehgal SN (1991) Pharmacology of rapamycin: a new immunosuppressive agent. Br J Rheumatol 30 Suppl 2:62-65.

Chang Q, Khare G, Dani V, et al (2006) The Disease Progression of Mecp2 Mutant Mice Is Affected by the Level of BDNF Expression. Neuron 49:341-348. doi: 10.1016/j.neuron.2005.12.027

Charrier C, Ehrensperger M-V, Dahan M, et al (2006) Cytoskeleton Regulation of Glycine Receptor Number at Synapses and Diffusion in the Plasma Membrane. J Neurosci 26:8502-8511. doi: 10.1523/JNEUROSCI.1758-06.2006

Chen J, Zheng XF, Brown EJ, Schreiber SL (1995) Identification of an 11-kDa FKBP12rapamycin-binding domain within the 289-kDa FKBP12-rapamycin-associated protein and characterization of a critical serine residue. Proc Natl Acad Sci U S A 92:49474951.

Chen Y, Mills JD, Periasamy A (2003) Protein localization in living cells and tissues using FRET and FLIM. Differ Res Biol Divers 71:528-541. doi: 10.1111/j.14320436.2003.07109007.x

Chiou T-T, Bonhomme B, Jin H, et al (2011) Differential Regulation of the Postsynaptic Clustering of -Aminobutyric Acid Type A (GABAA) Receptors by Collybistin Isoforms. J Biol Chem 286:22456-22468. doi: 10.1074/jbc.M111.236190

Chubykin AA, Atasoy D, Etherton MR, et al (2007) Activity-Dependent Validation of Excitatory versus Inhibitory Synapses by Neuroligin-1 versus Neuroligin-2. Neuron 54:919-931. doi: 10.1016/j.neuron.2007.05.029

Clegg RM (1995) Fluorescence resonance energy transfer. Curr Opin Biotechnol 6:103-110.

Cohen S, Gabel HW, Hemberg M, et al (2011) Genome-Wide Activity-Dependent MeCP2 Phosphorylation Regulates Nervous System Development and Function. Neuron 72:72-85. doi: 10.1016/j.neuron.2011.08.022 
Cole MJ, Siegel J, Webb SE, et al (2001) Time-domain whole-field fluorescence lifetime imaging with optical sectioning. J Microsc 203:246-257.

Colyer RA, Lee C, Gratton E (2008) A novel fluorescence lifetime imaging system that optimizes photon efficiency. Microsc Res Tech 71:201-213. doi: 10.1002/jemt.20540

Costa-Mattioli M, Monteggia LM (2013) mTOR complexes in neurodevelopmental and neuropsychiatric disorders. Nat Neurosci 16:1537-1543. doi: 10.1038/nn.3546

Cota D, Matter EK, Woods SC, Seeley RJ (2008) The Role of Hypothalamic Mammalian Target of Rapamycin Complex 1 Signaling in Diet-Induced Obesity. J Neurosci 28:7202-7208. doi: 10.1523/JNEUROSCI.1389-08.2008

Crews L, Spencer B, Desplats P, et al (2010) Selective Molecular Alterations in the Autophagy Pathway in Patients with Lewy Body Disease and in Models of $\alpha$ Synucleinopathy. PLoS ONE 5:e9313. doi: 10.1371/journal.pone.0009313

Crino PB, Nathanson KL, Henske EP (2006) The tuberous sclerosis complex. N Engl J Med 355:1345-1356. doi: 10.1056/NEJMra055323

Cunningham JT, Rodgers JT, Arlow DH, et al (2007) mTOR controls mitochondrial oxidative function through a YY1-PGC-1alpha transcriptional complex. Nature 450:736-740. doi: $10.1038 /$ nature06322

Dejanovic B, Semtner M, Ebert S, et al (2014) Palmitoylation of Gephyrin Controls Receptor Clustering and Plasticity of GABAergic Synapses. PLoS Biol 12:e1001908. doi: 10.1371/journal.pbio.1001908

Don Paul C, Kiss C, Traore DAK, et al (2013) Phanta: a non-fluorescent photochromic acceptor for pcFRET. PloS One 8:e75835. doi: 10.1371/journal.pone.0075835

Dotti CG, Sullivan CA, Banker GA (1988) The establishment of polarity by hippocampal neurons in culture. J Neurosci Off J Soc Neurosci 8:1454-1468.

Dowling K, Hyde SCW, Dainty JC, et al (1997) 2-D fluorescence lifetime imaging using a time-gated image intensifier. Opt Commun 135:27-31.

Dragich JM, Kim Y-H, Arnold AP, Schanen NC (2007) Differential distribution of the MeCP2 splice variants in the postnatal mouse brain. J Comp Neurol 501:526-542. doi: $10.1002 /$ cne. 21264

Duman RS, Li N, Liu R-J, et al (2012) Signaling pathways underlying the rapid antidepressant actions of ketamine. Neuropharmacology 62:35-41. doi: 10.1016/j.neuropharm.2011.08.044

Dumoulin A (2009) Cellular transport and membrane dynamics of the glycine receptor. Front Mol Neurosci. doi: 10.3389/neuro.02.028.2009

Dunlop EA, Tee AR (2014) mTOR and autophagy: A dynamic relationship governed by nutrients and energy. Semin Cell Dev Biol 36:121-129. doi: 10.1016/j.semcdb.2014.08.006

Durán RV, Hall MN (2012) Regulation of TOR by small GTPases. EMBO Rep 13:121-128. doi: 10.1038/embor.2011.257

Dymoke-Bradshaw AKL (1993) Impact of high-voltage pulse technology on high-speed photography. pp 2-6

Elson DS, Munro I, Requejo-Isidro J, et al (2004) Real-time time-domain fluorescence lifetime imaging including single-shot acquisition with a segmented optical image intensifier. New J Phys 6:180-180. doi: 10.1088/1367-2630/6/1/180

Esposito A, Dohm CP, Bähr M, Wouters FS (2007) Unsupervised Fluorescence Lifetime Imaging Microscopy for High Content and High Throughput Screening. Mol Amp Cell Proteomics 6:1446-1454. doi: 10.1074/mcp.T700006-MCP200 
Esposito A, Wouters FS (2004) Fluorescence lifetime imaging microscopy. Curr Protoc Cell Biol Editor Board Juan Bonifacino Al Chapter 4:Unit 4.14. doi: 10.1002/0471143030.cb0414s25

Fischer F, Kneussel M, Tintrup H, et al (2000) Reduced synaptic clustering of GABA and glycine receptors in the retina of the gephyrin null mutant mouse. J Comp Neurol 427:634-648.

Förster T (1948) Zwischenmolekulare Energiewanderung und Fluoreszenz. Ann Phys 437:55-75. doi: 10.1002/andp.19484370105

Fritschy J-M, Harvey RJ, Schwarz G (2008) Gephyrin: where do we stand, where do we go? Trends Neurosci 31:257-264. doi: 10.1016/j.tins.2008.02.006

Fritschy J-M, Panzanelli P, Tyagarajan SK (2012) Molecular and functional heterogeneity of GABAergic synapses. Cell Mol Life Sci 69:2485-2499. doi: 10.1007/s00018-0120926-4

Fukata Y, Fukata M (2010) Protein palmitoylation in neuronal development and synaptic plasticity. Nat Rev Neurosci 11:161-175. doi: 10.1038/nrn2788

Ganesan S, Ameer-Beg SM, Ng TTC, et al (2006) A dark yellow fluorescent protein (YFP)based Resonance Energy-Accepting Chromoprotein (REACh) for Förster resonance energy transfer with GFP. Proc Natl Acad Sci U S A 103:4089-4094. doi: 10.1073/pnas.0509922103

Gibson JR, Huber KM, Sudhof TC (2009) Neuroligin-2 Deletion Selectively Decreases Inhibitory Synaptic Transmission Originating from Fast-Spiking but Not from Somatostatin-Positive Interneurons. J Neurosci 29:13883-13897. doi: 10.1523/JNEUROSCI.2457-09.2009

Goedhart J, von Stetten D, Noirclerc-Savoye M, et al (2012) Structure-guided evolution of cyan fluorescent proteins towards a quantum yield of $93 \%$. Nat Commun 3:751. doi: 10.1038/ncomms 1738

Guertin DA, Stevens DM, Thoreen CC, et al (2006) Ablation in Mice of the mTORC Components raptor, rictor, or mLST8 Reveals that mTORC2 Is Required for Signaling to Akt-FOXO and PKCa, but Not S6K1. Dev Cell 11:859-871. doi: 10.1016/j.devcel.2006.10.007

Günther U, Benson J, Benke D, et al (1995) Benzodiazepine-insensitive mice generated by targeted disruption of the gamma 2 subunit gene of gamma-aminobutyric acid type $A$ receptors. Proc Natl Acad Sci 92:7749-7753.

Guy J, Hendrich B, Holmes M, et al (2001) A mouse Mecp2-null mutation causes neurological symptoms that mimic Rett syndrome. Nat Genet 27:322-326. doi: $10.1038 / 85899$

Hagberg B, Aicardi J, Dias K, Ramos O (1983) A progressive syndrome of autism, dementia, ataxia, and loss of purposeful hand use in girls: Rett's syndrome: Report of 35 cases. Ann Neurol 14:471-479. doi: 10.1002/ana.410140412

Hanus C (2004) Intracellular Association of Glycine Receptor with Gephyrin Increases Its Plasma Membrane Accumulation Rate. J Neurosci 24:1119-1128. doi: 10.1523/JNEUROSCI.4380-03.2004

Harvey K (2004) The GDP-GTP Exchange Factor Collybistin: An Essential Determinant of Neuronal Gephyrin Clustering. J Neurosci 24:5816-5826. doi: 10.1523/JNEUROSCI.1184-04.2004

Hawasli AH, Benavides DR, Nguyen C, et al (2007) Cyclin-dependent kinase 5 governs learning and synaptic plasticity via control of NMDAR degradation. Nat Neurosci 10:880-886. doi: 10.1038/nn1914 
Hay N (2004) Upstream and downstream of mTOR. Genes Dev 18:1926-1945. doi: 10.1101/gad.1212704

He L, Wu X, Simone J, et al (2005) Determination of tumor necrosis factor receptorassociated factor trimerization in living cells by CFP->YFP->mRFP FRET detected by flow cytometry. Nucleic Acids Res 33:e61. doi: 10.1093/nar/gni057

Helliwell SB, Wagner P, Kunz J, et al (1994) TOR1 and TOR2 are structurally and functionally similar but not identical phosphatidylinositol kinase homologues in yeast. Mol Biol Cell 5:105-118. doi: 10.1091/mbc.5.1.105

Hernandez-Negrete I, Carretero-Ortega J, Rosenfeldt H, et al (2007) P-Rex1 Links Mammalian Target of Rapamycin Signaling to Rac Activation and Cell Migration. J Biol Chem 282:23708-23715. doi: 10.1074/jbc.M703771200

Herweg J, Schwarz G (2012) Splice-specific Glycine Receptor Binding, Folding, and Phosphorylation of the Scaffolding Protein Gephyrin. J Biol Chem 287:12645-12656. doi: 10.1074/jbc.M112.341826

Hoeffer CA, Klann E (2010) mTOR signaling: At the crossroads of plasticity, memory and disease. Trends Neurosci 33:67-75. doi: 10.1016/j.tins.2009.11.003

Hoon M, Bauer G, Fritschy J-M, et al (2009) Neuroligin 2 Controls the Maturation of GABAergic Synapses and Information Processing in the Retina. J Neurosci 29:80398050. doi: 10.1523/JNEUROSCI.0534-09.2009

Hoon M, Soykan T, Falkenburger B, et al (2011) Neuroligin-4 is localized to glycinergic postsynapses and regulates inhibition in the retina. Proc Natl Acad Sci 108:30533058. doi: 10.1073/pnas.1006946108

Howell KR, Kutiyanawalla A, Pillai A (2011) Long-Term Continuous Corticosterone Treatment Decreases VEGF Receptor-2 Expression in Frontal Cortex. PLoS ONE 6:e20198. doi: 10.1371/journal.pone.0020198

$\mathrm{Hu}$ X, Luo J, Xu J (2015) The interplay between synaptic activity and neuroligin function in the CNS. BioMed Res Int 2015:498957. doi: 10.1155/2015/498957

Huang J, Manning BD (2008) The TSC1-TSC2 complex: a molecular switchboard controlling cell growth. Biochem J 412:179-190. doi: 10.1042/BJ20080281

Huang X, Zhang H, Yang J, et al (2010) Pharmacological inhibition of the mammalian target of rapamycin pathway suppresses acquired epilepsy. Neurobiol Dis 40:193-199. doi: 10.1016/j.nbd.2010.05.024

Hudson CC, Liu M, Chiang GG, et al (2002) Regulation of Hypoxia-Inducible Factor 1 Expression and Function by the Mammalian Target of Rapamycin. Mol Cell Biol 22:7004-7014. doi: 10.1128/MCB.22.20.7004-7014.2002

Hur E-M, Zhou F-Q (2010) GSK3 signalling in neural development. Nat Rev Neurosci 11:539-551. doi: 10.1038/nrn2870

Inoki K, Corradetti MN, Guan K-L (2005) Dysregulation of the TSC-mTOR pathway in human disease. Nat Genet 37:19-24. doi: 10.1038/ng1494

Inoki K, Zhu T, Guan K-L (2003) TSC2 mediates cellular energy response to control cell growth and survival. Cell 115:577-590.

Jedlicka P, Hoon M, Papadopoulos T, et al (2011) Increased Dentate Gyrus Excitability in Neuroligin-2-Deficient Mice in Vivo. Cereb Cortex 21:357-367. doi: 10.1093/cercor/bhq100

Ji Y, Pang PT, Feng L, Lu B (2005) Cyclic AMP controls BDNF-induced TrkB phosphorylation and dendritic spine formation in mature hippocampal neurons. Nat Neurosci 8:164-172. doi: 10.1038/nn1381 
Julien L-A, Carriere A, Moreau J, Roux PP (2010) mTORC1-Activated S6K1 Phosphorylates Rictor on Threonine 1135 and Regulates mTORC2 Signaling. Mol Cell Biol 30:908921. doi: 10.1128/MCB.00601-09

Kamimura Y, Xiong Y, Iglesias PA, et al (2008) PIP3-Independent Activation of TorC2 and PKB at the Cell's Leading Edge Mediates Chemotaxis. Curr Biol 18:1034-1043. doi: 10.1016/j.cub.2008.06.068

Kang R, Wan J, Arstikaitis $P$, et al (2008) Neural palmitoyl-proteomics reveals dynamic synaptic palmitoylation. Nature 456:904-909. doi: 10.1038/nature07605

Katsoulidou V, Bergmann A, Becker W (2007) How fast can TCSPC FLIM be made? $p$ 67710B-67710B-7

Kim EY, Schrader N, Smolinsky B, et al (2006) Deciphering the structural framework of glycine receptor anchoring by gephyrin. EMBO J 25:1385-1395.

Kim HW, Ha SH, Lee MN, et al (2010) Cyclic AMP Controls mTOR through Regulation of the Dynamic Interaction between Rheb and Phosphodiesterase 4D. Mol Cell Biol 30:5406-5420. doi: 10.1128/MCB.00217-10

Kins S, Betz H, Kirsch J (2000) Collybistin, a newly identified brain-specific GEF, induces submembrane clustering of gephyrin. Nat Neurosci 3:22-29. doi: 10.1038/71096

Kirsch J, Langosch D, Prior P, et al (1991) The 93-kDa glycine receptor-associated protein binds to tubulin. J Biol Chem 266:22242-22245.

Kneussel M, Betz H (2000) Receptors, gephyrin and gephyrin-associated proteins: novel insights into the assembly of inhibitory postsynaptic membrane specializations. J Physiol 525:1-9.

Kneussel M, Helmut Brandstätter J, Gasnier B, et al (2001) Gephyrin-Independent Clustering of Postsynaptic GABAA Receptor Subtypes. Mol Cell Neurosci 17:973-982. doi: 10.1006/mcne.2001.0983

Körber C, Richter A, Kaiser M, et al (2012) Effects of distinct collybistin isoforms on the formation of GABAergic synapses in hippocampal neurons. Mol Cell Neurosci 50:250-259. doi: 10.1016/j.mcn.2012.05.006

Kowalczyk S, Winkelmann A, Smolinsky B, et al (2013) Direct binding of GABA $A$ receptor $\beta 2$ and $\beta 3$ subunits to gephyrin. Eur J Neurosci 37:544-554. doi: 10.1111/ejn.12078

Kuhse J, Kalbouneh H, Schlicksupp A, et al (2012) Phosphorylation of Gephyrin in Hippocampal Neurons by Cyclin-dependent Kinase CDK5 at Ser-270 Is Dependent on Collybistin. J Biol Chem 287:30952-30966. doi: 10.1074/jbc.M112.349597

Lakowicz JR (ed) (2006) Principles of Fluorescence Spectroscopy. Springer US, Boston, MA

Laplante M, Sabatini DM (2012) mTOR Signaling in Growth Control and Disease. Cell 149:274-293. doi: 10.1016/j.cell.2012.03.017

Lardi-Studler B, Smolinsky B, Petitjean CM, et al (2007) Vertebrate-specific sequences in the gephyrin E-domain regulate cytosolic aggregation and postsynaptic clustering. J Cell Sci 120:1371-1382. doi: 10.1242/jcs.003905

Lebrun-Julien F, Bachmann L, Norrmen C, et al (2014) Balanced mTORC1 Activity in Oligodendrocytes Is Required for Accurate CNS Myelination. J Neurosci 34:84328448. doi: 10.1523/JNEUROSCI.1105-14.2014

Lee DY (2015) Roles of mTOR Signaling in Brain Development. Exp Neurobiol 24:177. doi: 10.5607/en.2015.24.3.177

Lee S, Comer FI, Sasaki A, et al (2005) TOR complex 2 integrates cell movement during chemotaxis and signal relay in Dictyostelium. Mol Biol Cell 16:4572-4583. doi: 10.1091/mbc.E05-04-0342 
Levi S (2004) Gephyrin Is Critical for Glycine Receptor Clustering But Not for the Formation of Functional GABAergic Synapses in Hippocampal Neurons. J Neurosci 24:207217. doi: 10.1523/JNEUROSCI.1661-03.2004

Lewis JD, Meehan RR, Henzel WJ, et al (1992) Purification, sequence, and cellular localization of a novel chromosomal protein that binds to Methylated DNA. Cell 69:905-914. doi: 10.1016/0092-8674(92)90610-O

Li B-S, Sun M-K, Zhang L, et al (2001) Regulation of NMDA receptors by cyclin-dependent kinase-5. Proc Natl Acad Sci 98:12742-12747. doi: 10.1073/pnas.211428098

Li M, Zhao L, Liu J, et al (2010) Multi-mechanisms are involved in reactive oxygen species regulation of mTORC1 signaling. Cell Signal 22:1469-1476. doi: 10.1016/j.cellsig.2010.05.015

Liu K, Lu Y, Lee JK, et al (2010) PTEN deletion enhances the regenerative ability of adult corticospinal neurons. Nat Neurosci 13:1075-1081. doi: 10.1038/nn.2603

Long X, Lin Y, Ortiz-Vega S, et al (2005) Rheb binds and regulates the mTOR kinase. Curr Biol CB 15:702-713. doi: 10.1016/j.cub.2005.02.053

Luscher B, Fuchs T, Kilpatrick CL (2011) GABAA Receptor Trafficking-Mediated Plasticity of Inhibitory Synapses. Neuron 70:385-409. doi: 10.1016/j.neuron.2011.03.024

Maas C, Tagnaouti N, Loebrich S, et al (2006) Neuronal cotransport of glycine receptor and the scaffold protein gephyrin. J Cell Biol 172:441-451. doi: 10.1083/jcb.200506066

Machado COF, Griesi-Oliveira K, Rosenberg C, et al (2015) Collybistin binds and inhibits mTORC1 signaling: a potential novel mechanism contributing to intellectual disability and autism.

Machado P, Rostaing P, Guigonis J-M, et al (2011) Heat Shock Cognate Protein 70 Regulates Gephyrin Clustering. J Neurosci 31:3-14. doi: 10.1523/JNEUROSCI.253310.2011

Markwardt ML, Kremers G-J, Kraft CA, et al (2011) An improved cerulean fluorescent protein with enhanced brightness and reduced reversible photoswitching. PloS One 6:e17896. doi: 10.1371/journal.pone.0017896

Meehan RR, Lewis JD, McKay S, et al (1989) Identification of a mammalian protein that binds specifically to DNA containing methylated CpGs. Cell 58:499-507.

Meyer G, Kirsch J, Betz H, Langosch D (1995) Identification of a gephyrin binding motif on the glycine receptor beta subunit. Neuron 15:563-572.

Miller MB, Yan Y, Eipper BA, Mains RE (2013) Neuronal Rho GEFs in synaptic physiology and behavior. The Neuroscientist 1073858413475486.

Miralvès J, Magdeleine E, Joly E (2007) Design of an improved set of oligonucleotide primers for genotyping MeCP2tm1.1Bird KO mice by PCR. Mol Neurodegener 2:16. doi: 10.1186/1750-1326-2-16

Morabito MA (2004) Cyclin-Dependent Kinase 5 Phosphorylates the N-Terminal Domain of the Postsynaptic Density Protein PSD-95 in Neurons. J Neurosci 24:865-876. doi: 10.1523/JNEUROSCI.4582-03.2004

Moretto Zita M, Marchionni I, Bottos E, et al (2007) Post-phosphorylation prolyl isomerisation of gephyrin represents a mechanism to modulate glycine receptors function. EMBO J 26:1761-1771. doi: 10.1038/sj.emboj.7601625

Mukherjee J, Kretschmannova K, Gouzer G, et al (2011) The residence time of GABA(A)Rs at inhibitory synapses is determined by direct binding of the receptor $\alpha 1$ subunit to gephyrin. J Neurosci Off J Soc Neurosci 31:14677-14687. doi: 10.1523/JNEUROSCI.2001-11.2011 
Nawrotzki R, Islinger M, Vogel I, et al (2012) Expression and subcellular distribution of gephyrin in non-neuronal tissues and cells. Histochem Cell Biol 137:471-482. doi: 10.1007/s00418-012-0914-7

O'Connor DV (1984) Time-correlated single photon counting. Academic Press, London; Orlando

Ogino K, Ramsden SL, Keib N, et al (2011) Duplicated Gephyrin Genes Showing Distinct Tissue Distribution and Alternative Splicing Patterns Mediate Molybdenum Cofactor Biosynthesis, Glycine Receptor Clustering, and Escape Behavior in Zebrafish. J Biol Chem 286:806-817. doi: 10.1074/jbc.M110.125500

Papadopoulos T, Eulenburg V, Reddy-Alla S, et al (2008) Collybistin is required for both the formation and maintenance of GABAergic postsynapses in the hippocampus. Mol Cell Neurosci 39:161-169. doi: 10.1016/j.mcn.2008.06.006

Patterson GH, Piston DW, Barisas BG (2000) Förster distances between green fluorescent protein pairs. Anal Biochem 284:438-440. doi: 10.1006/abio.2000.4708

Pelka GJ, Watson CM, Radziewic T, et al (2006) Mecp2 deficiency is associated with learning and cognitive deficits and altered gene activity in the hippocampal region of mice. Brain J Neurol 129:887-898. doi: 10.1093/brain/awl022

Petchprayoon C, Marriott G (2010) Synthesis and spectroscopic characterization of redshifted spironaphthoxazine based optical switch probes. Tetrahedron Lett 51:67535755. doi: 10.1016/j.tetlet.2010.10.084

Peterson TR, Sengupta SS, Harris TE, et al (2011) mTOR Complex 1 Regulates Lipin 1 Localization to Control the SREBP Pathway. Cell 146:408-420. doi: 10.1016/j.cell.2011.06.034

Pfeiffer F, Graham D, Betz H (1982) Purification by affinity chromatography of the glycine receptor of rat spinal cord. J Biol Chem 257:9389-9393.

Plenge RM, Stevenson RA, Lubs HA, et al (2002) Skewed X-Chromosome Inactivation Is a Common Feature of X-Linked Mental Retardation Disorders. Am J Hum Genet 71:168-173. doi: $10.1086 / 341123$

Poo M (2001) Neurotrophins as synaptic modulators. Nat Rev Neurosci 2:24-32. doi: $10.1038 / 35049004$

Poulopoulos A, Aramuni G, Meyer G, et al (2009) Neuroligin 2 Drives Postsynaptic Assembly at Perisomatic Inhibitory Synapses through Gephyrin and Collybistin. Neuron 63:628642. doi: 10.1016/j.neuron.2009.08.023

Rowley MI, Barber PR, Coolen ACC, Vojnovic B (2011) Bayesian analysis of fluorescence lifetime imaging data. pp 790325-790325-12

Rudrabhatla P, Pant HC (2010) Phosphorylation-specific peptidyl-prolyl isomerization of neuronal cytoskeletal proteins by Pin1: implications for therapeutics in neurodegeneration. J Alzheimers Dis JAD 19:389-403. doi: 10.3233/JAD-2010-1243

Russo E, Follesa P, Citraro R, et al (2014) The mTOR signaling pathway and neuronal stem/progenitor cell proliferation in the hippocampus are altered during the development of absence epilepsy in a genetic animal model. Neurol Sci 35:17931799. doi: 10.1007/s10072-014-1842-1

Sabatini DM, Barrow RK, Blackshaw S, et al (1999) Interaction of RAFT1 with gephyrin required for rapamycin-sensitive signaling. Science 284:1161-1164.

Sancak Y, Bar-Peled L, Zoncu R, et al (2010) Ragulator-Rag Complex Targets mTORC1 to the Lysosomal Surface and Is Necessary for Its Activation by Amino Acids. Cell 141:290-303. doi: 10.1016/j.cell.2010.02.024 
Sander B, Tria G, Shkumatov AV, et al (2013) Structural characterization of gephyrin by AFM and SAXS reveals a mixture of compact and extended states. Acta Crystallogr D Biol Crystallogr 69:2050-2060. doi: 10.1107/S0907444913018714

Sarbassov DD (2005) Phosphorylation and Regulation of Akt/PKB by the Rictor-mTOR Complex. Science 307:1098-1101. doi: 10.1126/science.1106148

Sassoe-Pognetto M, Kirsch J, Grünert U, et al (1995) Colocalization of gephyrin and GABAAreceptor subunits in the rat retina. J Comp Neurol 357:1-14.

Sassoè-Pognetto M, Panzanelli P, Sieghart W, Fritschy J-M (2000) Colocalization of multiple GABAA receptor subtypes with gephyrin at postsynaptic sites. J Comp Neurol 420:481-498.

Schrader N, Kim EY, Winking J, et al (2004) Biochemical Characterization of the High Affinity Binding between the Glycine Receptor and Gephyrin. J Biol Chem 279:18733-18741. doi: $10.1074 / j b c . M 311245200$

Schwarz G, Mendel RR, Ribbe MW (2009) Molybdenum cofactors, enzymes and pathways. Nature 460:839-847. doi: 10.1038/nature08302

Schwarz G, Schrader N, Mendel RR, et al (2001) Crystal structures of human gephyrin and plant $\mathrm{Cnx1} \mathrm{G}$ domains: comparative analysis and functional implications. J Mol Biol 312:405-418. doi: 10.1006/jmbi.2001.4952

Scully AD, MacRobert AJ, Botchway S, et al (1996) Development of a laser-based fluorescence microscope with subnanosecond time resolution. J Fluoresc 6:119-125.

Scully AD, Ostler RB, Phillips D, et al (1997) Application of fluorescence lifetime imaging microscopy to the investigation of intracellular PDT mechanisms. Bioimaging 5:9-18. doi: 10.1002/1361-6374(199703)5:1<9::AID-BIO2>3.0.CO;2-A

Schreiber KH, Kennedy BK et al. (2015) Rapamycin-mediated mTORC2 inhibition is determined by relative expression of FK506-binding proteins. Aging Cell 14(2):26573.

Shahbazian MD, Zoghbi HY (2001) Molecular genetics of Rett syndrome and clinical spectrum of MECP2 mutations. Curr Opin Neurol 14:171-176.

Smolinsky B, Eichler SA, Buchmeier S, et al (2008) Splice-specific Functions of Gephyrin in Molybdenum Cofactor Biosynthesis. J Biol Chem 283:17370-17379. doi: 10.1074/jbc.M800985200

Smrt RD, Eaves-Egenes J, Barkho BZ, et al (2007) Mecp2 deficiency leads to delayed maturation and altered gene expression in hippocampal neurons. Neurobiol Dis 27:77-89. doi: 10.1016/j.nbd.2007.04.005

Sola M, Bavro VN, Timmins J, et al (2004) Structural basis of dynamic glycine receptor clustering by gephyrin. EMBO J 23:2510-2519.

Soykan T, Schneeberger D, Tria G, et al (2014) A conformational switch in collybistin determines the differentiation of inhibitory postsynapses. EMBO J 33:2113-2133. doi: 10.15252/embj.201488143

Specht CG, Izeddin I, Rodriguez PC, et al (2013) Quantitative Nanoscopy of Inhibitory Synapses: Counting Gephyrin Molecules and Receptor Binding Sites. Neuron 79:308-321. doi: 10.1016/j.neuron.2013.05.013

Stallmeyer B, Schwarz G, Schulze J, et al (1999) The neurotransmitter receptor-anchoring protein gephyrin reconstitutes molybdenum cofactor biosynthesis in bacteria, plants, and mammalian cells. Proc Natl Acad Sci 96:1333-1338.

Su SC, Tsai L-H (2011) Cyclin-Dependent Kinases in Brain Development and Disease. Annu Rev Cell Dev Biol 27:465-491. doi: 10.1146/annurev-cellbio-092910-154023 
Subach FV, Zhang L, Gadella TWJ, et al (2010) Red fluorescent protein with reversibly photoswitchable absorbance for photochromic FRET. Chem Biol 17:745-755. doi: 10.1016/j.chembiol.2010.05.022

Tanaka J -i., Horiike Y, Matsuzaki M, et al (2008) Protein Synthesis and NeurotrophinDependent Structural Plasticity of Single Dendritic Spines. Science 319:1683-1687. doi: 10.1126/science.1152864

Tang G, Gudsnuk K, Kuo S-H, et al (2014) Loss of mTOR-Dependent Macroautophagy Causes Autistic-like Synaptic Pruning Deficits. Neuron 83:1131-1143. doi: 10.1016/j.neuron.2014.07.040

Tavazoie SF, Alvarez VA, Ridenour DA, et al (2005) Regulation of neuronal morphology and function by the tumor suppressors Tsc1 and Tsc2. Nat Neurosci 8:1727-1734. doi: $10.1038 / \mathrm{nn} 1566$

Thomanetz V, Angliker N, Cloetta D, et al (2013) Ablation of the mTORC2 component rictor in brain or Purkinje cells affects size and neuron morphology. J Cell Biol 201:293308. doi: $10.1083 / j c b .201205030$

Thomas GM, Huganir RL (2004) MAPK cascade signalling and synaptic plasticity. Nat Rev Neurosci 5:173-183. doi: 10.1038/nrn1346

Thoreen CC, Chantranupong L, Keys HR, et al (2012) A unifying model for mTORC1mediated regulation of mRNA translation. Nature 485:109-113. doi: 10.1038/nature11083

Tretter V, Jacob TC, Mukherjee J, et al (2008) The Clustering of GABAA Receptor Subtypes at Inhibitory Synapses is Facilitated via the Direct Binding of Receptor 2 Subunits to Gephyrin. J Neurosci 28:1356-1365. doi: 10.1523/JNEUROSCI.5050-07.2008

Tretter V, Kerschner B, Milenkovic I, et al (2011) Molecular Basis of the -Aminobutyric Acid A Receptor 3 Subunit Interaction with the Clustering Protein Gephyrin. J Biol Chem 286:37702-37711. doi: 10.1074/jbc.M111.291336

Tretter V, Mukherjee J, Maric H-M, et al (2012) Gephyrin, the enigmatic organizer at GABAergic synapses. Front Cell Neurosci. doi: 10.3389/fncel.2012.00023

Triller A, Cluzeaud F, Pfeiffer F, et al (1985) Distribution of glycine receptors at central synapses: an immunoelectron microscopy study. J Cell Biol 101:683-688.

Tyagarajan SK, Fritschy J-M (2014) Gephyrin: a master regulator of neuronal function? Nat Rev Neurosci 15:141-156. doi: 10.1038/nrn3670

Tyagarajan SK, Ghosh H, Harvey K, Fritschy J-M (2011) Collybistin splice variants differentially interact with gephyrin and Cdc42 to regulate gephyrin clustering at GABAergic synapses. J Cell Sci 124:2786-2796. doi: 10.1242/jcs.086199

Tyagarajan SK, Ghosh H, Yevenes GE, et al (2013) Extracellular Signal-regulated Kinase and Glycogen Synthase Kinase 3 Regulate Gephyrin Postsynaptic Aggregation and GABAergic Synaptic Function in a Calpain-dependent Mechanism. J Biol Chem 288:9634-9647. doi: 10.1074/jbc.M112.442616

Tyler WJ (2002) From Acquisition to Consolidation: On the Role of Brain-Derived Neurotrophic Factor Signaling in Hippocampal-Dependent Learning. Learn Mem 9:224-237. doi: 10.1101/lm.51202

Tyler WJ, Pozzo-Miller LD (2001) BDNF enhances quantal neurotransmitter release and increases the number of docked vesicles at the active zones of hippocampal excitatory synapses. J Neurosci Off J Soc Neurosci 21:4249-4258.

Ueno M, Carvalheira JBC, Tambascia RC, et al (2005) Regulation of insulin signalling by hyperinsulinaemia: role of IRS-1/2 serine phosphorylation and the mTOR/p70 S6K pathway. Diabetologia 48:506-518. doi: 10.1007/s00125-004-1662-6 
Van Munster EB, Gadella TWJ (2005) Fluorescence lifetime imaging microscopy (FLIM). Adv Biochem Eng Biotechnol 95:143-175.

Varoqueaux F, Jamain S, Brose N (2004) Neuroligin 2 is exclusively localized to inhibitory synapses. Eur J Cell Biol 83:449-456. doi: 10.1078/0171-9335-00410

Vecsler M, Simon AJ, Amariglio N, et al (2010) MeCP2 deficiency downregulates specific nuclear proteins that could be partially recovered by valproic acid in vitro. Epigenetics 5:61-67.

Wahl SE, McLane LE, Bercury KK, et al (2014) Mammalian Target of Rapamycin Promotes Oligodendrocyte Differentiation, Initiation and Extent of CNS Myelination. J Neurosci 34:4453-4465. doi: 10.1523/JNEUROSCI.4311-13.2014

Wang XF, Periasamy A, Herman B, Coleman DM (1992) Fluorescence Lifetime Imaging Microscopy (FLIM): Instrumentation and Applications. Crit Rev Anal Chem 23:369395. doi: 10.1080/10408349208051651

Wang XF, Uchida T, Coleman DM, Minami S (1991) A Two-Dimensional Fluorescence Lifetime Imaging System Using a Gated Image Intensifier. Appl Spectrosc 45:360366.

Weaving LS, Ellaway CJ, Gécz J, Christodoulou J (2005) Rett syndrome: clinical review and genetic update. J Med Genet 42:1-7. doi: 10.1136/jmg.2004.027730

Weiwad M, Küllertz G, Schutkowski M, Fischer G (2000) Evidence that the substrate backbone conformation is critical to phosphorylation by p42 MAP kinase. FEBS Lett 478:39-42. doi: 10.1016/S0014-5793(00)01794-4

Westmark PR, Westmark CJ, Wang S, et al (2010) Pin1 and PKM Sequentially Control Dendritic Protein Synthesis. Sci Signal 3:ra18-ra18. doi: 10.1126/scisignal.2000451

Wu P, Brand L (1994) Resonance energy transfer: methods and applications. Anal Biochem 218:1-13.

Wu X, Wu Z, Ning G, et al (2012) -Aminobutyric Acid Type A (GABAA) Receptor Subunits Play a Direct Role in Synaptic Versus Extrasynaptic Targeting. J Biol Chem 287:27417-27430. doi: 10.1074/jbc.M112.360461

Xiang S, Kim EY, Connelly JJ, et al (2006) The Crystal Structure of Cdc42 in Complex with Collybistin II, a Gephyrin-interacting Guanine Nucleotide Exchange Factor. J Mol Biol 359:35-46. doi: 10.1016/j.jmb.2006.03.019

Yaffe MB (1997) Sequence-Specific and Phosphorylation-Dependent Proline Isomerization: A Potential Mitotic Regulatory Mechanism. Science 278:1957-1960. doi: 10.1126/science.278.5345.1957

Yu J-J, Zhang Y, Wang Y, et al (2013) Inhibition of calcineurin in the prefrontal cortex induced depressive-like behavior through mTOR signaling pathway. Psychopharmacology (Berl) 225:361-372. doi: 10.1007/s00213-012-2823-9

Zeng L-H, Rensing NR, Wong M (2009) The Mammalian Target of Rapamycin Signaling Pathway Mediates Epileptogenesis in a Model of Temporal Lobe Epilepsy. J Neurosci 29:6964-6972. doi: 10.1523/JNEUROSCI.0066-09.2009

Zhang J, Gao Z, Yin J, et al (2008) S6K Directly Phosphorylates IRS-1 on Ser-270 to Promote Insulin Resistance in Response to TNF- Signaling through IKK2. J Biol Chem 283:35375-35382. doi: 10.1074/jbc.M806480200

Zhang J, Ji F, Liu Y, et al (2014) Ezh2 Regulates Adult Hippocampal Neurogenesis and Memory. J Neurosci 34:5184-5199. doi: 10.1523/JNEUROSCI.4129-13.2014

Zhou J, Tan S-H, Nicolas V, et al (2013) Activation of lysosomal function in the course of autophagy via mTORC1 suppression and autophagosome-lysosome fusion. Cell Res 23:508-523. doi: 10.1038/cr.2013.11 
Zhou X (2000) Pin1-Dependent Prolyl Isomerization Regulates Dephosphorylation of Cdc25C and Tau Proteins. Mol Cell 6:873-883. doi: 10.1016/S1097-2765(00)00085$\mathrm{X}$

Zinzalla V, Stracka D, Oppliger W, Hall MN (2011) Activation of mTORC2 by Association with the Ribosome. Cell 144:757-768. doi: 10.1016/j.cell.2011.02.014

Zoncu R, Bar-Peled L, Efeyan A, et al (2011) mTORC1 Senses Lysosomal Amino Acids Through an Inside-Out Mechanism That Requires the Vacuolar H+-ATPase. Science 334:678-683. doi: 10.1126/science.1207056

Zuccato C, Cattaneo E (2009) Brain-derived neurotrophic factor in neurodegenerative diseases. Nat Rev Neurol 5:311-322. doi: 10.1038/nrneurol.2009.54 


\section{Acknowledgements}

Firstly, I would like to express my sincere gratitude to my advisor Prof. Dr. Fred Wouters for the continuous support of my PhD study, for his patience, motivation and immense knowledge.

I also also want to thank my thesis committee, Dr. Till Marquardt and Prof. Jörg Enderlein, for their great response, open door and the opportunity for collaborations.

My genuine thanks go to Dr Gertrude Bunt for being a great teacher and for giving me the opportunity to finish my diploma thesis in her lab.

This thesis would never have seen completion if not for the continued moral, scientific and technical support of Dr. Craig Don Paul who is a true friend and amazing colleague.

I would like to thank Dr. Anastasia Loman, Ute Eilers, Mirja Fülle and Robert Ventzki for the time in the lab. Thanks go to Tina Ghelani for teaching me neuronal cell culture and for the great time in Chicago.

Finally I owe my deepest gratitude to my parents Hans-Rüdiger Ebrecht and Ingrid Ebrecht who always supported me. I want to thank my beloved sister Diana Mingram and her son Sam Mingram for being awesome. 


\section{Curriculum Vitae}

Name: $\quad$ René Ebrecht

Date of birth: $\quad 30$. October 1980

Place of birth: Magdeburg, Germany

Nationality: German

Martital status: Single

\section{Education:}

04.2012 - present University of Göttingen, DFG PhD Program "Molecular Physiology of the Brain" (Göttingen Graduate School for Neuroscience, Biophysics and Molecular Bioscience)

Thesis: "The signal transduction of synapse formation and it's failure in Rett syndrome" Supervisor: Prof. Dr. Fred S. Wouters

10. 2001 - 07.2010 Diplom in "Technical Biology", University of Stuttgart

Thesis: "Focal adhesion kinase in the regulation of cellular tension"

Supervisor: Prof. Dr. Klaus Pfizenmaier

09.1987 - 07.1999 Europaschule Gymnasium Gommern

\section{Additional Qualifications}

Languages German (native speaker), English (fluent), Russian (basic)

\section{Publications:}

Ebrecht R., Don Paul C., Wouters F.S. (2014) Fluorescence lifetime imaging microscopy in the medical sciences. Protoplasma 


\section{Posters:}

1) Optics within the life sciences 2012 ; Ebrecht R., Loman A., Gregor I., Enderlin J., Wouters F.S., Bunt G. "Probing the Connectivity of the Cellular signaling network by spectral and lifetime pattern-based ratiometric imaging"

2) Neurizons 2013 PhD Conference; Ebrecht R., Loman A., Gregor I., Enderlin J., Wouters F.S., Bunt G. "Probing the Connectivity of the Cellular signaling network by spectral and lifetime pattern-based ratiometric imaging"

3) Focus on Microscopy 2015; Ebrecht R., Bunt G., Wouters F.S. "FRET/FLIM imaging of the mTOR-Gephyrin signaling axis for synapse formation"

4) Society for Neuroscience meeting 2015; Ghelani T., Göttfert F., Ebrecht R., Wouters F., Wittenmayer N., Dresbach T. "A novel tool for active zone protein orientation: Dually lagged full length mRFP Bassoon mGFP protein" 